\title{
Empirical Investigation of Herding under Different Economic Setups
}

\author{
Ashish Kumar, K. N. Badhani \\ Indian Institute of Management Kashipur, Kashipur, India \\ Email: ashish.kumar@iimkashipur.ac.in,knbadhain@iimkashipur.ac.in
}

How to cite this paper: Kumar, A. and Badhani, K.N. (2018) Empirical Investigation of Herding under Different Economic Setups. Theoretical Economics Letters, 8, 3313-3352.

https://doi.org/10.4236/tel.2018.815205

Received: September 25, 2018

Accepted: November 23, 2018

Published: November 26, 2018

Copyright (c) 2018 by authors and Scientific Research Publishing Inc. This work is licensed under the Creative Commons Attribution International License (CC BY 4.0).

http://creativecommons.org/licenses/by/4.0/

\section{(c) (i) Open Access}

\begin{abstract}
Present study is an attempt to analyse the presence of herding in different economic conditions. A mix of developed and developing countries is selected from different corners of the word. Our sample comprises 35 world markets, out of which 18 are emerging markets while 17 are developed markets. Daily data of all constituents stocks of the representative indices of these markets are extracted over most recent period ranging from Jan. 2000 to Apr. 2018. Applying different methodologies static and time varying, we find that only 11 markets out of 35 exhibit significant herding behaviour. These markets majorly belong to Asia, Africa and Middle East. We also try to relate herding with region, culture and state of economy and do not find any significant relation of these variables with herding.
\end{abstract}

\section{Keywords}

Herding, Developed Economics, Developing Economics, CSAD

\section{Introduction}

Herding has been a popular subject of empirical investigation among researchers for the last two decades as it has been accepted as one of the explanations for failure of neo-classical asset pricing theories. Pochea et al. [1] describe the herding behaviour as investors' tendency to mimic other investors' action or follow the market consensus. This behaviour leads the investors to suppress their own belief and follow the others' action which may aggravate market volatility that creates instability in the market [2] [3] [4] [5] [6]. Blasco et al. [5] also report that herding has a linear relation with volatility which may be used in volatility forecasting. Chattopadhyay et al. [7] prove the predictability of the herd formation in financial markets which may provide a base for portfolio management strategy and help investors to earn handsome return in short run [8]. These fea- 
tures of the herding indicate presence of informational inefficiency in these stock markets. Gelos and Wei [9] and Ali et al. [10] state that emerging markets are comparative less informationally efficient as a result these markets are more prone to herding. Same is supported by Chang et al. [11]. They check the significance of herding over five stock markets and find no evidence of herding in US and Hong Kong markets, partial evidence for Japanese and significant evidence in South Korean and Taiwan stock markets.

Similar results are drawn by various studies conducted over emerging and developed markets in recent time period i.e. Alemanni and Ornelas [12] study nine emerging market all across the globe and find strong herding in all nine markets. Holmes et al. [13] study Portugal market, Economou et al. [14] analyse Athens stock market, Juan et al. [15] and Sharma et al. [16] check Chinese markets, Bhaduri et al. [17] and Chattopadhyay et al. [7] test Indian market while Lu et al. [18] investigate herding in Taiwan stock exchange and all find strong presence of herding in these emerging market. In contrast Gavriilidis et al. [19] study Spanish market, Wylie [20] and Galariotis et al. [21] analyse UK stock markets, Kremer and Nautz [22] test German stock exchanges and Bensaida [23] and Lee et al. [24] check US stock bourses and either find no evidence or a weak evidence of herding in these developed market.

A few researchers try to analyse the herding over a group of countries i.e. Blasco and Ferreruela [25] analyse the herding effect in 7 advanced countries including Germany, United Kingdom, United States, Mexico, Japan, Spain and France and find only herding evidence in Spanish market. Chiang and Zheng [26] study 18 international markets over a long period ranging from 1988 to 2009 and conclude with mixed results. They find six advance advanced markets and Asian markets are affected with significant herding while no evidence of herding is found in Latin American Markets. Economou et al. [27] tested PIGS (Portugal, Ireland, Greece and Spain) markets and find some herding evidence in Ireland and Greece while no herding is detected in Spain. In case of Portugal results are mixed. Gebka and Wohar [28] analyse herding over 32 countries on aggregate and sectorial level and not find any significance of herding at international level, although in case of some emerging countries results are mixed. Balcilar et al. [29] find strong evidence of herding over all Gulf Arab stock markets using regime-switching, smooth transition regression model. Mobarek et al. [6] report herding in European countries in asymmetric market conditions. Specifically they find herding in these countries during market crisis. Chang and Lin [30] evaluate 50 counties across the world and find significant herding in 18 countries. Economou et al. [31] analyse herding among four Euronext member countries and find herding in all four countries in post-merger period. Guney et al. [32] investigate herding in eight African markets and find presence of herding across all eight markets which they attributed to low transparency prevalent in those markets. Zheng et al. [33] also analyse nine Asian stock markets using daily data over various industries and find that in most of the countries herding is more pronounced during down markets and low trading volume. Kabir and 
Shakur [34] examine herding in Asian and Latin American stock exchanges across the different market states and find majority of the Asian markets suffering from herding over the different return, volatility and volume regime.

\section{Literature Review}

Although, a huge literature is available on herding in emerging markets and developed markets but no conclusive evidence is found yet. Present study is an attempt in the same directions but having some unique features. First, Majority of the studies covered either one or two region but we try to cover all corners of the globe. For the sake of analysis, we classify financial markets on regional basis and evolve with five regions: America and Latin America, Europe, Asia and Asia pacific, Africa and Middle East. Thereafter, we select most prominent markets over these five regions, that helps us focus on the comparative analysis of herding worldwide. Second, Low liquidity, weak regulations, speculative trading creates high information asymmetry in emerging markets [1], which makes them more prone to herding. In order to check this hypothesis, we select prominent advanced and emerging markets from each region based on the list provided by MSCI, so that we can compare the herd phenomena among developed and emerging market within a region and across the region. We also try to find the impact of the state of economy on herding which has not been explored by other researchers. Third, irrational behaviour of the investors is one of the prime reasons of herd creation in a market. Irrationality among investors and level of informational efficiency in a stock market may be affected by either external environment or inner psychology [30], which depends on the local culture of the country or region. We are covering different nations under our study, so it will be interesting to study the impact of a nation's culture on herding. Therefore, we try to find out the impact of the region and a nation's culture on herding which has not been attempted yet at world level. Fourth, herding may arise due to different market frictions. Liquidity black holes is one of the prominent reasons [6]. In order to avoid that problem, unlike the other studies, we focused on the comparative analysis of herding between developed and emerging markets using the liquid constituent indices of each country. In other words, stock index of each country poses most liquid stocks listed in that market which help us to avoid the problem of informational asymmetry and lack of arbitrage opportunity which is a usual feature of less traded market or stock. That will make our results more robust. Fifth, majority of the studies focused on static measure of herding while a very few are based on dynamic measure herding. In our paper, we are calculating both of the measures which help us to determine the long run or short run nature of herding across developed and emerging markets. We also attempt to analysis the possibility of asymmetric herding behaviour in selected market under different market conditions extensively. We analyse herding druing up market or down market, high and low volatility and high and low trading volume. OLS estimators are based on the mean as measure of location 
and ignore the information about the tail of the distribution. Therefore, we also use the quantile regression to find out herding under different market conditions.

Previewing our results, we find evidence of reverse herding or anti-herding in all American and Latin American markets barring US and Portugal. In case of Portugal, we find significant herding across all market scenarios up or down while in case of US overall no herding has been detected while presence of herding is indicated in case of declining market which is not statistically significant. In case of Asia and Asia-Pacific region, significant anti-herding measure is reported in case of all developed markets i.e. Australia, Hong Kong, Japan, New Zealand and South Korea. Among the developed markets, Singapore emerges as an exception where we find herding that is not statistically significant. Among emerging markets like China, India, Malaysia and Taiwan in the same region, Malaysia is an exception where significant anti herd measure prevails irrespective of market conditions while there is no asymmetry in herding in India and China over different market conditions. Among selected African markets, Kenyan and South African markets are two markets where herding prevails while in case of middle east all five sampled markets are suffering from herding. Our results do not find any significant relation between region, culture and state of economy with herding.

The remaining paper is organised as follows. Section 2 presents the empirical structure of the paper to detect the herding in selected markets while Section 3 describe the data and summary statistics. Section 4 reports the empirical evidence of herding behaviour over different market regime and Section 5 concludes.

\section{Methodology}

In behavioural finance, primarily there are two categories of methodologies to detect herding in any stock market. First type of methods are based on the observed investment behaviour of a specific category of investor either individual investors or a group of investors. Co-movement in their observed investment pattern is termed as herding. While, second type of methods focus on detection of herding assuming market as a whole and determine the presence of herding on market level rather than investors level. In case of first method, we require the details information of every transaction done by the selected category of the investors that generally suffers from misidentification of investors or infrequent data observations [22]. Even, it is very difficult to find of that sort of data for emerging markets. In case of second method, market price of the stocks under consideration is used for to detect herding, which is very commonly available. Therefore, our study is focusing on second type of methods which is based on the dispersion of stocks' return.

Christie and Huang [35] proposed first dispersion based measure of herding popularly known as cross sectional standard deviation of stock returns (CSSD). 
The basic idea of calculating CSSD is to check the dispersion of individual stock return around the market return during different phases of the market. The reasoning is that during extreme market movements investors have the tendency to suppress their own believe and tend to invest on the collective action of the market as a result value of CSSD will be low which is perceived as indication of herding. CSSD is expressed as follows:

$$
\operatorname{CSSD}_{t}=\left(\frac{1}{N} \sum_{i=1}^{N}\left(R_{i t}-R_{m t}\right)^{2}\right)^{1 / 2}
$$

where, $R_{i t}$ is return of the individual stock $i$ at time $t$, while $R_{m t}$ is the average of the returns of the all individual stock considered form that market at $t$ time. As our study is based on market indices, hence we have to consider index value as the market portfolio. Tan et al. [36] and Economou et al. [27] suggested no difference in results based on value weighted and equally weighted average of market return, therefore for sake of convenience, we use equally weighted portfolio return as market return.

Christie and Huang [35] approach suffers from a few drawbacks i.e. herding is studied under the conditions of extreme returns only while it may be prevailing over the entire return distribution, but become more prevalent during periods of market stress [35]. Economou et al. [27] also suggests the CSSD may be suffers from outliers. In light of the above arguments, we use an alternate method specified by Chang et al. [11] to measure herding.

Chang et al. [11] calculate herding based on cross section absolute dispersion of stock returns (CSAD) using same argument. They define CSAD as follows:

$$
\operatorname{CSAD}_{t}=\frac{1}{N} \sum_{i=1}^{N}\left|R_{i t}-R_{m t}\right|
$$

Chang et al. [11] argue that in case of rational market, market return and CSAD will have a positive and linear relation as suggested by CAPM. In the presence of market consensus, when extreme market movement happen this relation is expected to become non-linear. If in that time period investors tend to mimic each other as a result the CSAD will go down hence the relation between the square return and CSAD will be negative. That negative relation will be considered as an indication of herding. Same can be presented through following regression equation:

$$
C S A D_{t}=\gamma_{0}+\gamma_{1} \cdot\left|R_{m t}\right|+\gamma_{2} \cdot R_{m t}^{2}+\varepsilon_{t}
$$

If herding is present $\gamma_{2}$ must have a significant negative value [11].

\section{Data and Summary Statistics}

As specified earlier, we classify the whole word in five segments: America and Latin America, Europe, Asia and Asia pacific, Africa and Middle East. In American and Latin American region, we select Brazil, Canada, Chile, Mexico and United States; from Europe we sample Belgium, France, Germany, Greece, Netherland, Portugal, Russia, Sweden, Switzerland and United Kingdom. Australia, 
China, Hong Kong, India, Japan, Malaysia, New Zealand, Singapore, South Korea and Taiwan are selected from Asia and Asia Pacific region. Egypt, Kenya, Mauritius, Nigeria and South Africa are representing African region while Abu Dhabi, Bahrain, Qatar, Saudi Arabia and UAE are selected from Middle-East. In total, we select 35 stock markets all across the world and some are listed in Table 1 with the name of index considered for that country. Information on the state of stock market is provided by MSCI, we use the same to define a market as advanced or emerging. In order to avoid the problem of informational asymmetry and lack of arbitrage opportunity, we use stock price data of all constituent stocks of selected indices. These stocks are highly liquid stocks of the respective stock markets. Herding is perceived to be a short lived phenomenon [35], hence we use daily data and our data set comprises the daily stock price of constituent stocks of the selected stock indices, market capitalisation and trading volume of selected market. The sample period of our study is Jan. 2000 to April 2018. Herding is prevalent more in market downturn [35] thus our sample period is perfect to analysis herding as it comprise dotcom bubble bursting phase of early 2000, bullish stock market of 2002, 2008-09 subprime crisis, US debt crisis of 2011, Eurozone debt crisis 2010 and Chinese down turn of 2016.

The search subject of our study includes 35 stock markets all across the world. Data for the Middle East markets are not available for whole sample period that create a trade-off between number of countries and data length. We want representation of all parts of the world, hence we prefer to include more number of countries rather than large sample length. Thus, starting dates for some of the markets vary from other markets. We calculate daily stock return using adjusted closing price of the stocks as $R_{i t}=\ln \left(P_{t} / P_{t-1}\right) * 100$, where $P_{t}$ denotes the price of the stock $i$ at time $t$ while $P_{t-1}$ is price of the same stock at previous day. All the data used in our study is extracted from Bloomberg database.

Table 2 provides the summary statistics of CSAD and Market Return $\left(R_{M}\right)$ for all selected 35 stock markets. Out of our sample as per MSCI 18 are emerging markets while 17 are developed markets. By checking the mean value of CSAD and $R_{M}$, Saudi Arabia has the highest value of both while lowest value of CSAD and $R_{M}$ belong to Switzerland and Netherland, respectively. Higher value of CSAD suggests the higher variation among the returns of the selected stocks which indicate higher amount of volatility. If we analyse region wise, Middle East markets have highest average of CSAD (0.2074) and $R_{M}(0.0059)$ while lowest values of both are coming from Europe $(0.0119,0.0004)$. If we compare among the emerging and advanced markets, emerging markets average CSAD is 0.0254 which is almost double than the average CSAD value of.0138 of advanced market. If we compare them in terms of average daily market return, emerging countries average stock market daily return is four time higher than the advance markets. Average market return for emerging market is 0.0028 while the figure for advanced market is.0006. These results confirms that advanced countries' stock markets are more robust where unusual cross sectional variation among the stock returns is less. All the series are stationary at level. 
Table 1. Details of sample.

\begin{tabular}{|c|c|c|c|c|}
\hline Country Name & Stock Exchange & Index Name & State of Economy & Sample Period \\
\hline \multicolumn{5}{|c|}{ American and Latin American Markets } \\
\hline Brazil & Sao Paulo Stock Exchange & IBOVESPA Brazil Sao Paulo Stock Exchange Index & Emerging & Jan. 2000 to Apr. 2018 \\
\hline Canada & Toronto Stock Exchange & S\&P/TSX Composite Index & Developed & Jan. 2000 to Apr. 2018 \\
\hline Chile & Santiago Stock Exchange & IPSA Index & Emerging & Jan. 2000 to Apr. 2018 \\
\hline Mexico & Bolsa Mexicam de Valores & S\&P.BMV IPC & Emerging & Jan. 2000 to Apr. 2018 \\
\hline United States & NADAQ & Nasdaq Composite Index & Developed & Jan. 2000 to Apr. 2018 \\
\hline \multicolumn{5}{|c|}{ European Markets } \\
\hline Belgium & Brussels Stock Exchange & BEL 20 Index & Developed & Jan. 2000 to Apr. 2018 \\
\hline France & French Stock Exchange & CAC40 Index & Developed & Jan. 2000 to Apr. 2018 \\
\hline Germany & Frankfurt Stock Exchange & Deutsche Boerse AG German Stock Index & Developed & Jan. 2000 to Apr. 2018 \\
\hline Greece & Athens Stock Exchange & Athens Stock Exchange General Index & Developed & Jan. 2000 to Apr. 2018 \\
\hline Netherland & Amsterdam Exchange & AEX Index & Developed & Jan. 2000 to Apr. 2018 \\
\hline Portugal & Lisbon Stock Exchange & PSI20 Index & Developed & Jan. 2000 to Apr. 2018 \\
\hline Russia & Moscow Stock Exchange & MOEX Russia Index & Emerging & Jan. 2000 to Apr. 2018 \\
\hline Sweden & Stockholm Stock Exchange & OMX Stockholm 30 Index & Developed & Jan. 2000 to Apr. 2018 \\
\hline Switzerland & Swiss Stock Exchange (SIX) & Swiss Market Index & Developed & Jan. 2000 to Apr. 2018 \\
\hline United Kingdom & London Stock Exchange & FTSE 100 Index & Developed & Jan. 2000 to Apr. 2018 \\
\hline \multicolumn{5}{|c|}{ Asian and Asia Pacific Markets } \\
\hline Australia & Australian Stock Exchange & Australian Stock Exchange All Ordinaries Index & Developed & Jan. 2000 to Apr. 2018 \\
\hline China & Shanghai Stock Exchange & Shanghai Stock Exchange Composite Index & Emerging & Jan. 2000 to Apr. 2018 \\
\hline Hong Kong & $\begin{array}{l}\text { Stock Exchange } \\
\text { of Hong Kong }\end{array}$ & Hong Kong Hang Sang Index & Developed & Jan. 2000 to Apr. 2018 \\
\hline India & National Stock Exchange & CNX Nifty 50 & Emerging & Jan. 2000 to Apr. 2018 \\
\hline Japan & Tokyo Stock Exchange & Nikkei 225 & Developed & Jan. 2000 to Apr. 2018 \\
\hline Malaysia & $\begin{array}{l}\text { Kuala Lumpur } \\
\text { Stock Exchange }\end{array}$ & FTSE Bursa Malaysia KLCI Index & Emerging & Jan. 2000 to Apr. 2018 \\
\hline New Zealand & New Zealand Exchange Ltd. & S\&P/NZX All Index & Developed & Jan. 2000 to Apr. 2018 \\
\hline Singapore & Singapore Exchange & The Straits Times Index & Developed & Jan. 2000 to Apr. 2018 \\
\hline South Korea & Korean Stock Exchange & Korea Stock Exchange KOSPI Index & Developed & Jan. 2000 to Apr. 2018 \\
\hline Taiwan & Taiwan Stock Exchange & Taiwan Stock Exchange Weighted Index & Emerging & Jan. 2000 to Apr. 2018 \\
\hline \multicolumn{5}{|c|}{ African Markets } \\
\hline Egypt & $\begin{array}{l}\text { Cairo and Alexandria } \\
\text { Stock Exchange }\end{array}$ & EGX 30 Index & Emerging & Jan. 2000 to Apr. 2018 \\
\hline Kenya & Nairobi Security Exchange & Nairobi Securities Exchange Ltd. All Share Index & Emerging & Jan. 2000 to Apr. 2018 \\
\hline Mauritius & Mauritius Stock Exchange & Mauritius Stock Exchange SEMDEX Index & Emerging & Jan. 2000 to Apr. 2018 \\
\hline Nigeria & Lagos Stock Exchange & NSE 30 Index & Emerging & Jan. 2000 to Apr. 2018 \\
\hline South Africa & Johannesburg Stock Exchange & FTSE/JSE Africa All Share Index & Emerging & Jan. 2000 to Apr. 2018 \\
\hline \multicolumn{5}{|c|}{ Middle-East Markets } \\
\hline Abu Dhabi & Abu Dhabi Security Market & Abu Dhabi Security Market General Index & Emerging & Feb. 2002 to Apr. 2018 \\
\hline Bahrain & Bahrain Bourse & Bahrain Bourse All Share Index & Emerging & Feb. 2002 to Apr. 2018 \\
\hline Qatar & Qatar Exchange & Qatar Exchange Index & Emerging & Jan. 2003 to Apr. 2018 \\
\hline Saudi Arabia & Saudi Stock Exchange & Tadawul All Share Index & Emerging & Feb. 2002 to Apr. 2018 \\
\hline UAE & Dubai Financial Market & Dubai Financial Market General Index & Emerging & Feb. 2002 to Apr. 2018 \\
\hline
\end{tabular}


Table 2. Descriptive statistics.

\begin{tabular}{|c|c|c|c|c|c|c|c|c|c|}
\hline Country & Variables & Mean & Max. & Min. & SD & Kurtosis & Skewness & $\mathbf{N}$ & $\mathrm{ADF}$ \\
\hline \multicolumn{10}{|c|}{ American and Latin American Markets } \\
\hline \multirow{2}{*}{ Brazil } & CSAD & 0.0153 & 0.1080 & 0.0056 & 0.0052 & 3.5671 & 40.0954 & 4517 & $-9.2981^{*}$ \\
\hline & $R_{m}$ & 0.0010 & 0.1413 & -0.0916 & 0.0148 & 0.0508 & 8.4284 & 4517 & $-65.0513^{*}$ \\
\hline \multirow{2}{*}{ Canada } & CSAD & 0.0150 & 0.0699 & 0.0052 & 0.0055 & 2.3535 & 12.3679 & 4394 & $-4.9964^{*}$ \\
\hline & $R_{m}$ & 0.0007 & 0.0785 & -0.0647 & 0.0087 & -0.4241 & 10.7640 & 4394 & $-60.7506^{*}$ \\
\hline \multirow{2}{*}{ Chile } & CSAD & 0.0133 & 0.0606 & 0.0048 & 0.0050 & 1.6479 & 8.2668 & 4270 & $-9.0276^{*}$ \\
\hline & $R_{m}$ & 0.0004 & 0.1563 & -0.1152 & 0.0150 & -0.1855 & 10.5676 & 4270 & $-65.5883^{*}$ \\
\hline \multirow{2}{*}{ Mexico } & CSAD & 0.0123 & 0.0870 & 0.0026 & 0.0053 & 3.0119 & 24.7001 & 4419 & $-11.6347^{\star}$ \\
\hline & $R_{m}$ & 0.0008 & 0.1084 & -0.0605 & 0.0104 & -0.1195 & 9.4272 & 4419 & $-58.6922^{*}$ \\
\hline \multirow{2}{*}{ United States } & CSAD & 0.0153 & 0.0855 & 0.0040 & 0.0084 & 1.9221 & 7.7289 & 4399 & $-5.0629^{*}$ \\
\hline & $R_{m}$ & 0.0009 & 0.1102 & -0.0926 & 0.0159 & 0.1527 & 7.2469 & 4399 & $-67.7702^{*}$ \\
\hline \multicolumn{10}{|c|}{ European Markets } \\
\hline \multirow{2}{*}{ Belgium } & CSAD & 0.0107 & 0.0896 & 0.0008 & 0.0060 & 3.1794 & 21.5524 & 4364 & $-6.4416^{*}$ \\
\hline & $R_{m}$ & 0.0004 & 0.0988 & -0.0716 & 0.0117 & 0.0951 & 9.7564 & 4364 & $-40.2054^{\star}$ \\
\hline \multirow{2}{*}{ France } & CSAD & 0.0111 & 0.0439 & 0.0030 & 0.0054 & 1.7398 & 6.8787 & 4365 & $-5.5688^{\star}$ \\
\hline & $R_{m}$ & 0.0003 & 0.1105 & -0.0870 & 0.0142 & 0.0386 & 8.0858 & 4365 & $-65.0504^{*}$ \\
\hline \multirow{2}{*}{ Germany } & CSAD & 0.0158 & 0.1066 & 0.0026 & 0.0102 & 2.1732 & 9.9798 & 4251 & $-4.0983^{*}$ \\
\hline & $R_{m}$ & 0.0009 & 0.1247 & -0.0982 & 0.0182 & 0.0386 & 6.6848 & 4251 & $-65.7873^{*}$ \\
\hline \multirow{2}{*}{ Greece } & CSAD & 0.0181 & 2.0424 & 0.0063 & 0.0313 & 61.7241 & 3986.0340 & 4368 & $-64.2865^{\star}$ \\
\hline & $R_{m}$ & 0.0003 & 1.0497 & -0.1128 & 0.0231 & 21.4679 & 980.7622 & 4368 & $-62.5821^{\star}$ \\
\hline \multirow{2}{*}{ Netherland } & CSAD & 0.0124 & 0.0792 & 0.0030 & 0.0070 & 2.5444 & 14.4734 & 3734 & $-5.6986^{*}$ \\
\hline & $R_{m}$ & 0.0002 & 0.0859 & -0.0858 & 0.0138 & -0.1660 & 7.2037 & 3734 & $-60.2646^{*}$ \\
\hline \multirow{2}{*}{ Portugal } & CSAD & 0.0118 & 0.0453 & 0.0017 & 0.0051 & 1.1840 & 5.3883 & 4347 & $-8.9953^{*}$ \\
\hline & $\mathrm{R}_{\mathrm{m}}$ & 0.0003 & 0.1205 & -0.0851 & 0.0107 & -0.0701 & 10.8122 & 4347 & $-59.6797^{\star}$ \\
\hline \multirow{2}{*}{ Russia } & CSAD & 0.0175 & 2.7226 & 0.0059 & 0.0428 & 61.5696 & 3888.0710 & 4092 & $-62.2565^{\star}$ \\
\hline & $R_{m}$ & 0.0005 & 1.4032 & -0.1138 & 0.0280 & 30.6296 & 1535.6350 & 4092 & $-60.7592^{\star}$ \\
\hline \multirow{2}{*}{ Sweden } & CSAD & 0.0116 & 0.0544 & 0.0028 & 0.0057 & 1.8020 & 8.0114 & 4302 & $-6.6121^{*}$ \\
\hline & $R_{m}$ & 0.0005 & 0.0967 & -0.0846 & 0.0141 & 0.1142 & 8.0014 & 4302 & $-66.3752^{*}$ \\
\hline \multirow{2}{*}{ Switzerland } & CSAD & 0.0097 & 0.0678 & 0.0022 & 0.0056 & 2.4300 & 13.1977 & 4310 & $-6.0131^{*}$ \\
\hline & $R_{m}$ & 0.0003 & 0.1098 & -0.0959 & 0.0130 & -0.0347 & 9.5940 & 4310 & $-61.8773^{\star}$ \\
\hline \multirow{2}{*}{$\begin{array}{l}\text { United King- } \\
\text { dom }\end{array}$} & CSAD & 0.0118 & 0.0627 & 0.0037 & 0.0053 & 2.3744 & 11.6243 & 4314 & $-4.2095^{\star}$ \\
\hline & $R_{m}$ & 0.0005 & 0.0827 & -0.0792 & 0.0115 & -0.1298 & 8.3736 & 4314 & $-31.2532^{*}$ \\
\hline \multicolumn{10}{|c|}{ Asian and Asia Pacific Markets } \\
\hline \multirow{2}{*}{ Australia } & CSAD & 0.0195 & 0.0723 & 0.0108 & 0.0049 & 2.5997 & 15.7253 & 4446 & $-5.6732^{*}$ \\
\hline & $R_{m}$ & 0.0010 & 0.0572 & -0.0816 & 0.0075 & -1.2322 & 14.2363 & 4446 & $-40.1632^{\star}$ \\
\hline \multirow{2}{*}{ China } & CSAD & 0.0153 & 0.0554 & 0.0046 & 0.0063 & 1.6862 & 7.6508 & 4281 & $-7.2032^{\star}$ \\
\hline & $R_{m}$ & 0.0010 & 0.0973 & -0.0866 & 0.0168 & -0.3143 & 6.9555 & 4281 & $-62.2724^{*}$ \\
\hline \multirow{2}{*}{ Hong Kong } & CSAD & 0.0127 & 0.0798 & 0.0036 & 0.0054 & 2.6255 & 17.7104 & 4501 & $-7.1868^{\star}$ \\
\hline & $R_{m}$ & 0.0008 & 0.1232 & -0.1223 & 0.0141 & -0.1411 & 9.3815 & 4501 & $-64.0493^{\star}$ \\
\hline
\end{tabular}




\section{Continued}

\begin{tabular}{|c|c|c|c|c|c|c|c|c|c|}
\hline \multirow{2}{*}{ India } & CSAD & 0.0152 & 0.0655 & 0.0048 & 0.0063 & 2.0869 & 9.7373 & 4136 & $-7.9486^{\star}$ \\
\hline & $R_{m}$ & 0.0010 & 0.1499 & -0.1015 & 0.0136 & -0.2486 & 11.3726 & 4136 & $-57.1508^{\star}$ \\
\hline \multirow{2}{*}{ Japan } & CSAD & 0.0133 & 0.0484 & 0.0045 & 0.0050 & 1.5124 & 6.7622 & 4249 & $-6.4957^{\star}$ \\
\hline & $R_{m}$ & 0.0004 & 0.1563 & -0.1152 & 0.0150 & -0.2156 & 10.5976 & 4249 & $-65.2798^{\star}$ \\
\hline \multirow{2}{*}{ Malaysia } & CSAD & 0.0103 & 2.4953 & 0.0025 & 0.0389 & 63.1949 & 4038.7660 & 4131 & $-3.4816^{*}$ \\
\hline & $R_{m}$ & 0.0008 & 1.4205 & -0.0793 & 0.0235 & 53.4536 & 3233.1940 & 4131 & $-18.5887^{\star}$ \\
\hline \multirow{2}{*}{ New Zealand } & CSAD & 0.0152 & 0.2840 & 0.0043 & 0.0080 & 13.1520 & 359.6190 & 4415 & $-21.9298^{*}$ \\
\hline & $R_{m}$ & 0.0008 & 0.1437 & -0.0536 & 0.0067 & 3.1844 & 66.2039 & 4415 & $-63.0652^{\star}$ \\
\hline \multirow{2}{*}{ Singapore } & CSAD & 0.0119 & 0.0624 & 0.0036 & 0.0054 & 1.7976 & 9.0854 & 4300 & $-6.1125^{\star}$ \\
\hline & $R_{m}$ & 0.0004 & 0.0916 & -0.0787 & 0.0108 & -0.1405 & 9.6689 & 4300 & $-64.1093^{*}$ \\
\hline \multirow{2}{*}{ South Korea } & CSAD & 0.0183 & 0.2827 & 0.0071 & 0.0077 & 11.1406 & 335.9560 & 4355 & $-9.7787^{*}$ \\
\hline & $R_{m}$ & 0.0009 & 0.1522 & -0.1158 & 0.0144 & -0.2204 & 11.8386 & 4355 & $-63.7752^{*}$ \\
\hline \multirow{2}{*}{ Taiwan } & CSAD & 0.0157 & 0.1627 & 0.0027 & 0.0055 & 5.2681 & 120.7680 & 4342 & $-5.4553^{\star}$ \\
\hline & $R_{m}$ & 0.0005 & 0.0781 & -0.0812 & 0.0129 & -0.4766 & 7.0725 & 4342 & $-57.6735^{\star}$ \\
\hline \multicolumn{10}{|c|}{ African Markets } \\
\hline \multirow{2}{*}{ Egypt } & CSAD & 0.0166 & 0.2664 & 0.0036 & 0.0103 & 12.0824 & 268.9353 & 2582 & $-9.7329^{\star}$ \\
\hline & $R_{m}$ & 0.0016 & 0.1408 & -0.1173 & 0.0161 & -0.1824 & 11.1962 & 2582 & $-32.1238^{*}$ \\
\hline \multirow{2}{*}{ Kenya } & CSAD & 0.0415 & 0.4599 & 0.0032 & 0.0540 & 2.9300 & 13.7755 & 3345 & $-11.8875^{\star}$ \\
\hline & $R_{m}$ & 0.0052 & 0.6005 & -0.4297 & 0.0678 & 2.1065 & 24.2321 & 3345 & $-40.3585^{\star}$ \\
\hline \multirow{2}{*}{ Mauritius } & CSAD & 0.0115 & 10.9024 & 0.0003 & 0.1678 & 64.8047 & 4207.7160 & 4224 & $-64.2551^{*}$ \\
\hline & $R_{m}$ & 0.0021 & 5.7924 & -0.1105 & 0.0894 & 64.4109 & 4173.7490 & 4224 & $-65.6715^{*}$ \\
\hline \multirow{2}{*}{ Nigeria } & CSAD & 0.0496 & 2.4284 & 0.0022 & 0.0911 & 10.2444 & 204.9955 & 2943 & $-11.9514^{*}$ \\
\hline & $R_{m}$ & 0.0067 & 1.4452 & -0.3406 & 0.0838 & 5.6057 & 71.0684 & 2943 & $-35.2064^{\star}$ \\
\hline \multirow{2}{*}{ South Africa } & CSAD & 0.0151 & 0.0655 & 0.0054 & 0.0062 & 2.1403 & 10.2293 & 3918 & $-6.1535^{\star}$ \\
\hline & $R_{m}$ & 0.0009 & 0.1499 & -0.1015 & 0.0136 & -0.2251 & 11.6174 & 3918 & $-55.5471^{\star}$ \\
\hline \multicolumn{10}{|c|}{ Middle-East Markets } \\
\hline \multirow{2}{*}{ Abu Dhabi } & CSAD & 0.0451 & 0.5159 & 0.0002 & 0.0553 & 2.7692 & 13.7121 & 2186 & $-9.9988^{*}$ \\
\hline & $R_{m}$ & 0.0081 & 0.8667 & -0.3171 & 0.0661 & 2.7053 & 31.9677 & 2186 & $-36.7878^{*}$ \\
\hline \multirow{2}{*}{ Bahrain } & CSAD & 0.0331 & 0.3855 & 0.0001 & 0.0436 & 3.1420 & 16.0826 & 2264 & $-32.6562^{*}$ \\
\hline & $R_{m}$ & 0.0065 & 0.4103 & -0.2949 & 0.0518 & 1.7950 & 18.2189 & 2264 & $-45.7715^{\star}$ \\
\hline \multirow{2}{*}{ Qatar } & CSAD & 0.0319 & 0.6440 & 0.0022 & 0.0423 & 3.7020 & 32.5943 & 1941 & $-10.3015^{\star}$ \\
\hline & $R_{m}$ & 0.0063 & 1.1260 & -0.4967 & 0.0777 & 2.9543 & 41.5736 & 1941 & $-48.0686^{\star}$ \\
\hline \multirow{2}{*}{ Saudi Arabia } & CSAD & 0.0831 & 0.9598 & 0.0195 & 0.0759 & 4.0470 & 28.1256 & 2118 & $-31.9418^{\star}$ \\
\hline & $R_{m}$ & 0.0077 & 0.9425 & -0.5843 & 0.0877 & 1.5443 & 25.7858 & 2118 & $-46.8506^{\star}$ \\
\hline \multirow{2}{*}{ UAE } & CSAD & 0.0142 & 0.1791 & 0.0002 & 0.0092 & 4.7350 & 65.8020 & 2432 & $-9.4836^{*}$ \\
\hline & $R_{m}$ & 0.0009 & 0.1342 & -0.1032 & 0.0170 & -0.0237 & 12.6864 & 2432 & $-46.7657^{\star}$ \\
\hline
\end{tabular}

This table presents descriptive statistics of the daily cross-sectional absolute deviation (CSAD) and return of market portfolio $\left(R_{m}\right) . \quad C S A D_{t}=\frac{1}{N} \sum_{i=1}^{N}\left|R_{i t}-R_{m t}\right|$, where $R_{i t}$ is daily stock returns while $R_{m t}$ is equally weighted market returns. Sample period is Jan. 2000 to Apr. 2018. ${ }^{*}$ indicates significant at $1 \%$ level of significance. 


\section{Empricial Results}

\subsection{Estimation of Base Model of Herding}

We start our analysis by using the basic model of herding proposed by Chang et al. [11] using Equation (3). Table 3 shows the regression estimates of the model by country. A significant negative value of $\gamma_{2}$ indicates the presence of herding while significant positive value of same is a sign of the presence of anti-herding behaviour. If we look region wise, among all American and Latin American stock markets apart from US stock market have a significant positive estimate of $\gamma_{2}$ which shows strong anti-herding behaviour explained by Gebka and Wohar [28]. They state that when investors either overemphasize on their own view or excessively focus on the views of a subset of other market players that results in increased cross sectional dispersion and lead to anti-herding. In other words, the returns dispersion increases during market stress more than suggested by rational pricing model and that may be because of due to overconfidence or flight to quality [37]. In case of Europe, except for Portugal, all other European market irrespective of emerging and advanced, depict anti-herding behaviour. Portugal is the single European market in our sample that is observed with strong presence of herding. If we look at Asian and Asia Pacific markets, our results are more or less similar to Kabir and Shakur [34]. Among all emerging Asian markets barring Malaysia, all others are having strong form of herding while among all advanced Asian markets excluding Singapore all shown anti-herding behaviour. Similar to the results of Guney et al. [32], among African market only Kenya and South African markets reflect presence of herding while herding is highly prevalent in all five Middle-East markets. In case of Middle East our results confirm the results of Balcilar et al. [29].

\subsection{Estimation of Asymmetrical Nature of Herding during Up and Down Market}

Tan et al. [36] analyse investors' behaviour under different market conditions and find that they behave differently in up and down market. Thus, it may be possible that herding have asymmetrical nature under different market conditions too. Even, Christie and Huang [35] argue that herding is more prevalent during stress. Stavroyiannis and Babalos [37] also state that herding might be more pronounced during market turmoil, period of abnormal information flows or a period facing high volatility. In these cases, investors feel more comfortable with market consensus. Thus to check the asymmetric nature of herding, we reformulate Equation (3) by multiplying with days dummies.

$$
\begin{gathered}
\text { CSAD }_{t}=\gamma_{0}^{+}+\gamma_{1}^{+} \cdot\left|R_{m t}\right| \cdot \text { Dummy }_{U p}+\gamma_{2}^{+} \cdot R_{m t}^{2} \cdot \text { Dummy }_{U p}+\varepsilon_{t} \\
\text { CSAD }_{t}=\gamma_{0}^{-}+\gamma_{1}^{-} \cdot\left|R_{m t}\right| \cdot \text { Dummy }_{\text {Down }}+\gamma_{2}^{-} \cdot R_{m t}^{2} \cdot \text { Dummy }_{\text {Down }}+\varepsilon_{t}
\end{gathered}
$$

where, Dummy $y_{U p}$ is 1 when market return is positive else 0, while Dummy ${ }_{\text {Down }}$ is 1 when market return is negative else 0 . 
Table 3. Regression estimates of herding behaviour using base model.

\begin{tabular}{|c|c|c|c|c|}
\hline Country Name & $\gamma_{0}$ & $\gamma_{1}$ & $\gamma_{2}$ & Adjusted $\mathrm{R}^{2}$ \\
\hline \multicolumn{5}{|c|}{ American Markets } \\
\hline \multirow[t]{2}{*}{ Brazil } & $0.0127^{\star}$ & $0.1972^{\star}$ & $1.8596^{\star}$ & 33.36 \\
\hline & $(118.554)$ & $(17.896)$ & $(9.883)$ & \\
\hline \multirow[t]{2}{*}{ Canada } & $0.0122^{*}$ & $0.0482^{*}$ & $3.187^{*}$ & 34.67 \\
\hline & (111.595) & $(20.561)$ & $(6.016)$ & \\
\hline \multirow[t]{2}{*}{ Chile } & $0.0012^{\star}$ & $0.1885^{\star}$ & $0.3558^{* *}$ & 18.68 \\
\hline & $(98.287)$ & $(16.82)$ & $(2.052)$ & \\
\hline \multirow[t]{2}{*}{ Mexico } & $0.0092^{*}$ & $0.03742^{*}$ & $2.532^{*}$ & 41.83 \\
\hline & $(93.081)$ & $(25.103)$ & $(7.125)$ & \\
\hline \multirow[t]{2}{*}{ United States } & $0.0108^{*}$ & $0.3977^{*}$ & 0.2325 & 30.62 \\
\hline & $(62.214)$ & $(20.891)$ & $(0.672)$ & \\
\hline \multicolumn{5}{|c|}{ European Markets } \\
\hline \multirow[t]{2}{*}{ Belgium } & $0.0076^{*}$ & $0.3392^{*}$ & $2.4031^{\star}$ & 39.77 \\
\hline & $(67.863)$ & $(21.47)$ & $(7.177)$ & \\
\hline \multirow[t]{2}{*}{ France } & $0.0088^{*}$ & $0.2004^{*}$ & $1.2604^{*}$ & 24.15 \\
\hline & $(75.811)$ & $(14.632)$ & $(4.871)$ & \\
\hline \multirow[t]{2}{*}{ Germany } & $0.0085^{\star}$ & $0.2377^{\star}$ & $1.1005^{*}$ & 26.31 \\
\hline & $(72.325)$ & $(17.678)$ & $(4.495)$ & \\
\hline \multirow[t]{2}{*}{ Greece } & $0.0143^{*}$ & $24.97^{*}$ & $1.6019^{*}$ & 97.14 \\
\hline & (125.79) & $(35.430)$ & $(191.960)$ & \\
\hline \multirow[t]{2}{*}{ Netherland } & $0.0092^{*}$ & $0.2925^{\star}$ & $2.4083^{*}$ & 32.81 \\
\hline & $(57.000)$ & $(14.220)$ & $(5.680)$ & \\
\hline \multirow[t]{2}{*}{ Portugal } & $0.0087^{\star}$ & $0.4302^{*}$ & $-1.3357^{*}$ & 3.58 \\
\hline & $(87.749)$ & $(32.261)$ & $(-4.776)$ & \\
\hline \multirow[t]{2}{*}{ Russia } & $0.0132^{\star}$ & $0.2711^{*}$ & $1.1827^{\star}$ & 98.37 \\
\hline & $(116.6)$ & $(40.6)$ & $(215.6)$ & \\
\hline \multirow[t]{2}{*}{ Sweden } & $0.0091^{*}$ & $0.2374^{*}$ & $0.6006^{* *}$ & 21.80 \\
\hline & $(70.41)$ & $(15.4)$ & $(2.03)$ & \\
\hline \multirow[t]{2}{*}{ Switzerland } & $0.0069^{*}$ & $0.276^{*}$ & $1.6681^{\star}$ & 35.91 \\
\hline & $(62.892)$ & $(19.618)$ & $(6.181)$ & \\
\hline \multirow[t]{2}{*}{ United Kingdom } & $0.0095^{*}$ & $0.2383^{*}$ & $2.9244^{*}$ & 30.74 \\
\hline & $(86.524)$ & $(14.712)$ & $(7.843)$ & \\
\hline \multicolumn{5}{|c|}{ Asia Pacific Markets } \\
\hline \multirow[t]{2}{*}{ Australia } & $0.01659^{*}$ & $0.5458^{*}$ & $1.317^{*}$ & 41.12 \\
\hline & $(182.063)$ & $(31.122)$ & $(2.754)$ & \\
\hline \multirow[t]{2}{*}{ China } & $0.0118^{*}$ & $0.3409^{*}$ & $-1.9478^{\star}$ & 22.31 \\
\hline & $(81.69)$ & $(21.47)$ & $(-6.999)$ & \\
\hline \multirow[t]{2}{*}{ Hong Kong } & $0.0099^{*}$ & $0.2749^{*}$ & $0.7264^{*}$ & 33.39 \\
\hline & $(89.861)$ & $(22.63)$ & $(3.421)$ & \\
\hline
\end{tabular}




\section{Continued}

\begin{tabular}{|c|c|c|c|c|}
\hline \multirow[t]{2}{*}{ India } & $0.01178^{*}$ & $0.3703^{*}$ & $-0.7574^{\star}$ & 26.84 \\
\hline & $(87.781)$ & $(25.357)$ & $(-3.152)$ & \\
\hline \multirow[t]{2}{*}{ Japan } & $0.0112^{\star}$ & $0.1885^{\star}$ & $0.3558^{\star *}$ & 18.68 \\
\hline & (98.287) & $(16.682)$ & $(2.052)$ & \\
\hline \multirow[t]{2}{*}{ Malaysia } & $0.0072^{*}$ & $0.4383^{*}$ & $0.9245^{*}$ & 99.34 \\
\hline & $(107.67)$ & (51.73) & $(150.39)$ & \\
\hline \multirow[t]{2}{*}{ New Zealand } & $0.0197^{\star}$ & $0.8936^{*}$ & $7.719^{*}$ & 72.63 \\
\hline & $(120.53)$ & $(52.37)$ & $(31.86)$ & \\
\hline \multirow[t]{2}{*}{ Singapore } & $0.009^{*}$ & $0.3954^{*}$ & -0.3558 & 30.00 \\
\hline & $(79.355)$ & $(23.212)$ & $(0.912)$ & \\
\hline \multirow[t]{2}{*}{ South Korea } & $0.0157^{\star}$ & $0.1605^{*}$ & $5.0789^{*}$ & 40.69 \\
\hline & $(111.61)$ & $(10.77)$ & $(21.8)$ & \\
\hline \multirow[t]{2}{*}{ Taiwan } & $0.0125^{*}$ & $0.412^{*}$ & $-2.8279^{*}$ & 27.64 \\
\hline & $(104.87)$ & $(23.76)$ & $(-7.07)$ & \\
\hline \multicolumn{5}{|c|}{ African Markets } \\
\hline \multirow[t]{2}{*}{ Egypt } & $0.0154^{\star}$ & $-0.0815^{*}$ & $8.4261^{*}$ & 35.57 \\
\hline & $(57.665)$ & $(-3.264)$ & $(23.886)$ & \\
\hline \multirow[t]{2}{*}{ Kenya } & $0.0184^{*}$ & $1.0177^{\star}$ & $-0.9194^{\star}$ & 71.16 \\
\hline & $(30.07)$ & $(56.5)$ & $(-19.58)$ & \\
\hline \multirow[t]{2}{*}{ Mauritius } & $0.0045^{*}$ & $1.0407^{\star}$ & $0.1451^{*}$ & 99.94 \\
\hline & $(57.59)$ & $(89.82)$ & (72.48) & \\
\hline \multirow[t]{2}{*}{ Nigeria } & $0.0214^{*}$ & $0.8299^{*}$ & $0.2754^{*}$ & 76.10 \\
\hline & $(23.05)$ & $(47.12)$ & (11.54) & \\
\hline \multirow[t]{2}{*}{ South Africa } & $0.01177^{\star}$ & $0.3703^{*}$ & $-0.7574^{\star}$ & 26.84 \\
\hline & $(87.871)$ & $(25.387)$ & $(-3.152)$ & \\
\hline \multicolumn{5}{|c|}{ Middle-East Markets } \\
\hline \multirow[t]{2}{*}{ Abu Dhabi } & $0.01917^{\star}$ & $0.9792^{*}$ & $-0.7435^{\star}$ & 64.95 \\
\hline & $(23.09)$ & $(48.4)$ & $(-15.33)$ & \\
\hline \multirow[t]{2}{*}{ Bahrain } & $0.0129^{*}$ & $0.8126^{*}$ & $-0.1456^{*}$ & 66.01 \\
\hline & $(16.289)$ & $(38.064)$ & $(-2.995)$ & \\
\hline \multirow[t]{2}{*}{ Qatar } & $0.0149^{*}$ & $0.5216^{*}$ & $-0.0908^{\star}$ & 67.93 \\
\hline & $(22.33)$ & $(38.94)$ & $(-3.77)$ & \\
\hline \multirow[t]{2}{*}{ Saudi Arabia } & $0.0209^{*}$ & $0.5493^{*}$ & $-0.0599^{\star}$ & 62.71 \\
\hline & $(24.426)$ & $(40.94)$ & $(-3.739)$ & \\
\hline \multirow[t]{2}{*}{ UAE } & $0.0085^{\star}$ & $0.5939^{*}$ & $-2.7369^{*}$ & 38.64 \\
\hline & (39.208) & $(26.703)$ & $(-8.982)$ & \\
\hline
\end{tabular}

This table reports the estimated coefficients of the Equation (3) $\left(C S A D_{t}=\gamma_{0}+\gamma_{1} \cdot\left|R_{m t}\right|+\gamma_{2} \cdot R_{m t}^{2}+\varepsilon_{t}\right)$. $\mathrm{t}$-statistics are given in parentheses. A negative and significant value of $\gamma_{2}$ indicates the presence of herding in that market while a positively significant value is an indication of anti-herding. *indicates statistical significance at $1 \%$ level. ${ }^{\star *}$ indicates statistical significance at $5 \%$ level. 
Equation (4) try to analyse the relation of absolute market return and square of market return on the days when market return was positive and results are reported in Table 4. The negative and statistical significant estimates of $\gamma_{2}^{+}$ imply the presence of herding with market consensus by investors in the high return state. Barring few exceptions results of Equation (4) are more or less same to the results of Equation (3). Chile, Mexico and Greece has shown anti-herding behaviour in overall scenario but in case of up market positive significant coefficient turned insignificant. Opposite to that, Bahrain market is having negative significant coefficient in case of overall market while it is showing anti-herding behaviour during up market days. Taiwan and UAE are having strong herding in overall market while during up days both of the country's' coefficient turned positive but insignificant. Malaysia is the only country which have significant positive coefficient which turned into significant negative coefficient during up days.

Relation of absolute return and square market return with CSAD during negative return days are checked using Equation (5) and results are reported in Table 5. Investors herd under market stress or not that can be examined by estimate of $\gamma_{2}^{-}$. Similar to Chiang et al. [38], majority of the markets show nonlinearity in low and high market regimes. Except few countries those are having positive and significant coefficients of $\gamma_{2}$ in overall market and up market days now having insignificant coefficients during negative market returns. Interestingly, US market herding coefficient turned negative but still it is insignificant.

In order to establish whether this difference in up market days and down market days are statistically significant or not, we use a nested model presented in Equation (6).

$$
C S A D_{t}=\gamma_{0}+\gamma_{1} \cdot\left|R_{m t}\right|+\gamma_{2} \cdot R_{m t}^{2} \cdot \text { Dummy }_{U p}+\gamma_{3} \cdot R_{m t}^{2} \cdot \text { Dummy }_{\text {Down }}+\varepsilon_{t}
$$

We also apply Wald test to check the statically significance of the difference between $\gamma_{2}$ and $\gamma_{3}$ and results of the same are presented in Table 6. Similar to the results of the Galariotis et al. [21], we find statically significant difference between the estimates of $\gamma_{2}$ and $\gamma_{3}$ in all 35 cases that indicates the asymmetrical behaviour of investors during up days and down days all over the world.

\subsection{Augmented Model of Herding}

De Long et al. [39] argue that positive feedback traders buy in up market and sell in down market while negative feedback trader just do opposite of that. An arbitrageur is reluctant to trade again mispricing because of the unpredictability of the timings of the trend reversal. As a result due to dominance of noise traders, price become more volatile and cross sectional correlation rise in high volatile market [40]. Thus, $R_{m t}^{2}$ alone cannot capture the dynamics of nonlinearity [34]. Hence, we introduce $R_{m t}^{3}$ term in Equation (3) suggested by Chiang et al. [38] which is an interaction of herding behaviour present by $R_{m t}^{2}$ and market return presented by $R_{m t}$. Same is presented in Equation (7). 
Table 4. Regression estimates of herding behaviour in rising market.

\begin{tabular}{|c|c|c|c|c|}
\hline Country Name & $\gamma_{0}^{+}$ & $\gamma_{1}^{+}$ & $\gamma_{2}^{+}$ & Adjusted $\mathrm{R}^{2}$ \\
\hline \multicolumn{5}{|c|}{ American and Latin American Markets } \\
\hline \multirow[t]{2}{*}{ Brazil } & $0.0129^{*}$ & $0.1472^{*}$ & $3.3298^{*}$ & 29.82 \\
\hline & $(81.195)$ & $(8.01)$ & $(8.751)$ & \\
\hline \multirow[t]{2}{*}{ Canada } & $0.01227^{\star}$ & $0.4007^{*}$ & $3.792^{*}$ & 30.88 \\
\hline & $(85.055)$ & $(14.533)$ & $(3.792)$ & \\
\hline \multirow[t]{2}{*}{ Chile } & $0.0111^{\star}$ & $0.2056^{*}$ & 0.1099 & 20.06 \\
\hline & $(70.264)$ & $(13.646)$ & $(0.535)$ & \\
\hline \multirow[t]{2}{*}{ Mexico } & $0.0091^{*}$ & $0.4041^{\star}$ & 1.1008 & 36.22 \\
\hline & $(62.522)$ & $(15.389)$ & $(1.333)$ & \\
\hline \multirow[t]{2}{*}{ United States } & $0.0107^{*}$ & $0.3669^{*}$ & $1.0395^{\star *}$ & 30.19 \\
\hline & $(46.435)$ & $(13.943)$ & $(2.027)$ & \\
\hline \multicolumn{5}{|c|}{ European Markets } \\
\hline \multirow[t]{2}{*}{ Belgium } & $0.0073^{*}$ & $0.3528^{*}$ & $2.1974^{*}$ & 41.24 \\
\hline & $(50.833)$ & $(17.511)$ & $(5.606)$ & \\
\hline \multirow[t]{2}{*}{ France } & $0.0087^{\star}$ & $0.1914^{*}$ & $1.6619^{*}$ & 25.01 \\
\hline & $(54.719)$ & (10.13) & $(4.526)$ & \\
\hline \multirow[t]{2}{*}{ Germany } & $0.0087^{\star}$ & $0.1847^{*}$ & $2.3897^{*}$ & 24.06 \\
\hline & $(51.615)$ & (8.298) & $(4.877)$ & \\
\hline \multirow[t]{2}{*}{ Greece } & $0.0137^{\star}$ & $0.3206^{*}$ & 0.3291 & 37.15 \\
\hline & $(76.8)$ & (18.123) & (1.192) & \\
\hline \multirow[t]{2}{*}{ Netherland } & $0.0091^{\star}$ & $0.3163^{*}$ & $1.8114^{*}$ & 30.79 \\
\hline & $(35.221)$ & $(9.766)$ & $(2.745)$ & \\
\hline \multirow[t]{2}{*}{ Portugal } & $0.0085^{*}$ & $0.4222^{*}$ & $-1.1662^{*}$ & 34.04 \\
\hline & $(65.122)$ & $(24.609)$ & $(-3.625)$ & \\
\hline \multirow[t]{2}{*}{ Russia } & $0.0128^{*}$ & $0.3126^{*}$ & $0.5806^{\star *}$ & 39.84 \\
\hline & $(71.208)$ & $(18.587)$ & $(2.383)$ & \\
\hline \multirow[t]{2}{*}{ Sweden } & $0.0091^{*}$ & $0.2062^{*}$ & $1.841^{\star}$ & 21.71 \\
\hline & $(51.904)$ & $(9.755)$ & $(2.848)$ & \\
\hline \multirow[t]{2}{*}{ Switzerland } & $0.0069^{*}$ & $0.2468^{*}$ & $2.255^{*}$ & 34.40 \\
\hline & $(46.1)$ & $(11.51)$ & $(4.76)$ & \\
\hline \multirow[t]{2}{*}{ United Kingdom } & $0.0095^{\star}$ & $0.2146^{*}$ & $3.8109^{*}$ & 29.70 \\
\hline & $(65.007)$ & $(9.486)$ & $(6.358)$ & \\
\hline \multicolumn{5}{|c|}{ Asian and Asia Pacific Markets } \\
\hline \multirow[t]{2}{*}{ Australia } & $0.0166^{*}$ & $0.5024^{*}$ & $2.8081^{\star}$ & 39.22 \\
\hline & $(140.396)$ & $(20.579)$ & $(3.652)$ & \\
\hline \multirow[t]{2}{*}{ China } & $0.0119^{*}$ & $0.3249^{*}$ & $-1.2382^{*}$ & 24.55 \\
\hline & $(57.532)$ & $(14.2)$ & $(-2.812)$ & \\
\hline \multirow[t]{2}{*}{ Hong Kong } & $0.0103^{*}$ & $0.1927^{*}$ & $2.9106^{*}$ & 35.11 \\
\hline & $(64.074)$ & $(9.863)$ & $(7.376)$ & \\
\hline
\end{tabular}




\section{Continued}

\begin{tabular}{|c|c|c|c|c|}
\hline \multirow[t]{2}{*}{ India } & $0.01171^{\star}$ & $0.3589^{*}$ & $-0.7735^{\star}$ & 27.93 \\
\hline & $(66.517)$ & $(19.513)$ & $(-2.875)$ & \\
\hline \multirow[t]{2}{*}{ Japan } & $0.0111^{*}$ & $0.2056^{*}$ & 0.1099 & 20.06 \\
\hline & $(70.2664)$ & (13.646) & $(0.535)$ & \\
\hline \multirow[t]{2}{*}{ Malaysia } & $0.0072^{*}$ & $0.4258^{*}$ & $0.9334^{*}$ & 99.69 \\
\hline & (76.74) & (35.78) & (110.01) & \\
\hline \multirow[t]{2}{*}{ New Zealand } & $0.01104^{*}$ & $0.8285^{*}$ & $11.38^{*}$ & 70.26 \\
\hline & $(89.28)$ & (32.58) & $(21.31)$ & \\
\hline \multirow[t]{2}{*}{ Singapore } & $0.0089^{*}$ & $0.39^{*}$ & -0.4481 & 31.39 \\
\hline & (57.418) & $(17.411)$ & $(-0.981)$ & \\
\hline \multirow{2}{*}{ South Korea } & $0.01604^{*}$ & $0.0683^{*}$ & $7.6394^{*}$ & 51.05 \\
\hline & $(85.7)$ & (3.521) & (26.819) & \\
\hline \multirow[t]{2}{*}{ Taiwan } & $0.01286^{*}$ & $0.3077^{*}$ & 0.6063 & 28.55 \\
\hline & $(76.146)$ & $(12.669)$ & (1.102) & \\
\hline \multicolumn{5}{|c|}{ African Markets } \\
\hline \multirow[t]{2}{*}{ Egypt } & $0.0167^{*}$ & $-0.0298^{*}$ & $14.487^{*}$ & 50.26 \\
\hline & (32.64) & $(-6.36)$ & (21.09) & \\
\hline \multirow[t]{2}{*}{ Kenya } & $0.0182^{*}$ & $1.0358^{*}$ & $-0.0894^{*}$ & 70.12 \\
\hline & (16.97) & (32.76) & $(-11.34)$ & \\
\hline \multirow[t]{2}{*}{ Mauritius } & $0.0043^{*}$ & $1.1672^{*}$ & $-6.8322^{\star}$ & 64.77 \\
\hline & $(36.44)$ & $(42.84)$ & $(-8.85)$ & \\
\hline \multirow[t]{2}{*}{ Nigeria } & $0.0204^{*}$ & $0.837^{*}$ & $0.3222^{*}$ & 69.97 \\
\hline & $(14.801)$ & $(24.722)$ & (4.239) & \\
\hline \multirow[t]{2}{*}{ South Africa } & $0.01171^{\star}$ & $0.3589^{\star}$ & $-0.7735^{\star}$ & 27.93 \\
\hline & $(68.517)$ & $(19.513)$ & $(-2.875)$ & \\
\hline \multicolumn{5}{|c|}{ Middle-East Markets } \\
\hline \multirow[t]{2}{*}{ Abu Dhabi } & $0.0205^{*}$ & $1.0362^{*}$ & $-0.8632^{*}$ & 63.83 \\
\hline & $(10.985)$ & $(18.79)$ & $(-4.675)$ & \\
\hline \multirow[t]{2}{*}{ Bahrain } & $0.01735^{*}$ & $0.4813^{*}$ & $1.1788^{*}$ & 59.59 \\
\hline & $(10.547)$ & $(8.565)$ & $(5.991)$ & \\
\hline \multirow[t]{2}{*}{ Qatar } & $0.0108^{*}$ & $0.6746^{*}$ & $-0.5256^{*}$ & 72.28 \\
\hline & $(8.786)$ & $(21.878)$ & $(-7.179)$ & \\
\hline \multirow[t]{2}{*}{ Saudi Arabia } & $0.0217^{*}$ & $0.5216^{*}$ & -0.0124 & 68.00 \\
\hline & $(13.213)$ & $(21.472)$ & $(-0.586)$ & \\
\hline \multirow[t]{2}{*}{ UAE } & $0.0096^{*}$ & $0.3885^{*}$ & 1.1043 & 38.34 \\
\hline & (21.128) & (8.435) & (1.746) & \\
\hline
\end{tabular}

This table reports the estimated coefficients of the Equation (4)

$\operatorname{CSAD}_{t}=\gamma_{0}^{+}+\gamma_{1}^{+} \cdot\left|R_{m t}\right| \cdot$ Dummy $_{U p}+\gamma_{2}^{+} \cdot R_{m t}^{2} \cdot$ Dummy $_{U p}+\varepsilon_{t} \cdot \mathrm{t}$-statistics are given in parentheses. A negative and significant value of $\gamma_{2}^{+}$indicates the presence of herding in rising market while a positively significant value is an indication of anti-herding. ${ }^{*}$ indicates statistical significance at $1 \%$ level. ${ }^{* *}$ indicates statistical significance at $5 \%$ level. 
Table 5. Regression estimates of herding behaviour in declining market.

\begin{tabular}{|c|c|c|c|c|}
\hline Country Name & $\gamma_{0}^{-}$ & $\gamma_{1}^{-}$ & $\gamma_{2}^{-}$ & Adjusted $\mathbf{R}^{2}$ \\
\hline \multicolumn{5}{|c|}{ American and Latin American Markets } \\
\hline \multirow[t]{2}{*}{ Brazil } & $0.0126^{*}$ & $0.2297^{\star}$ & $1.0969^{*}$ & 35.94 \\
\hline & $(79.361)$ & $(14.836)$ & $(4.477)$ & \\
\hline \multirow[t]{2}{*}{ Canada } & $0.01221^{\star}$ & $0.4167^{\star}$ & $3.2009^{*}$ & 38.96 \\
\hline & $(68.768)$ & $(13.779)$ & $(4.344)$ & \\
\hline \multirow[t]{2}{*}{ Chile } & $0.0114^{*}$ & $0.1602^{*}$ & $0.7852^{\star *}$ & 15.82 \\
\hline & $(63.838)$ & $(8.173)$ & $(2.175)$ & \\
\hline \multirow[t]{2}{*}{ Mexico } & $0.0093^{*}$ & $0.3739^{*}$ & $2.8029^{*}$ & 47.66 \\
\hline & $(61.063)$ & $(17.793)$ & $(6.654)$ & \\
\hline \multirow[t]{2}{*}{ United States } & $0.0109^{*}$ & $0.4314^{*}$ & -0.6175 & 30.65 \\
\hline & $(40.188)$ & $(15.064)$ & $(-1.264)$ & \\
\hline \multicolumn{5}{|c|}{ European Markets } \\
\hline \multirow[t]{2}{*}{ Belgium } & $0.0079^{*}$ & $0.3156^{\star}$ & $2.6773^{*}$ & 37.76 \\
\hline & $(43.655)$ & $(11.712)$ & $(4.246)$ & \\
\hline \multirow[t]{2}{*}{ France } & $0.0089^{*}$ & $0.2076^{*}$ & $0.8467^{\star *}$ & 22.62 \\
\hline & (51.508) & $(10.222)$ & $(2.261)$ & \\
\hline \multirow[t]{2}{*}{ Germany } & $0.0085^{\star}$ & $0.2626^{*}$ & 0.6464 & 29.36 \\
\hline & $(48.277)$ & $(1.856)$ & $(0.295)$ & \\
\hline \multirow[t]{2}{*}{ Greece } & $0.0138^{*}$ & $0.3409^{*}$ & -0.3046 & 35.21 \\
\hline & $(62.308)$ & $(15.537)$ & $(-0.853)$ & \\
\hline \multirow[t]{2}{*}{ Netherland } & $0.0093^{*}$ & $0.2728^{*}$ & $2.4153^{*}$ & 29.08 \\
\hline & $(36.696)$ & $(8.108)$ & $(3.262)$ & \\
\hline \multirow[t]{2}{*}{ Portugal } & $0.0088^{*}$ & $0.4517^{*}$ & $-1.7718^{\star}$ & 33.76 \\
\hline & $(55.488)$ & $(19.346)$ & $(-3.059)$ & \\
\hline \multirow[t]{2}{*}{ Russia } & $0.0129^{*}$ & $0.3199^{*}$ & 0.1284 & 37.48 \\
\hline & $(58.92)$ & $(15.28)$ & $(0.39)$ & \\
\hline \multirow[t]{2}{*}{ Sweden } & $0.0092^{*}$ & $0.2682^{\star}$ & 0.0200 & 22.00 \\
\hline & $(46.141)$ & $(11.493)$ & $(0.046)$ & \\
\hline \multirow[t]{2}{*}{ Switzerland } & $0.0064^{*}$ & $0.3077^{\star}$ & $1.2278^{*}$ & 38.14 \\
\hline & (40.139) & $(14.898)$ & $(3.456)$ & \\
\hline \multirow[t]{2}{*}{ United Kingdom } & $0.0094^{*}$ & $0.2704^{\star}$ & $2.7319^{*}$ & 32.39 \\
\hline & $(55.039)$ & (10.759) & $(4.645)$ & \\
\hline \multicolumn{5}{|c|}{ Asian and Asia Pacific Markets } \\
\hline \multirow[t]{2}{*}{ Australia } & $0.0156^{*}$ & $0.5953^{*}$ & 0.1487 & 44.61 \\
\hline & $(109.52)$ & $(21.71)$ & $(0.23)$ & \\
\hline \multirow[t]{2}{*}{ China } & $0.0117^{\star}$ & $0.3342^{*}$ & $-2.1354^{\star}$ & 21.71 \\
\hline & (54.09) & (14.59) & $(-5.67)$ & \\
\hline
\end{tabular}




\section{Continued}

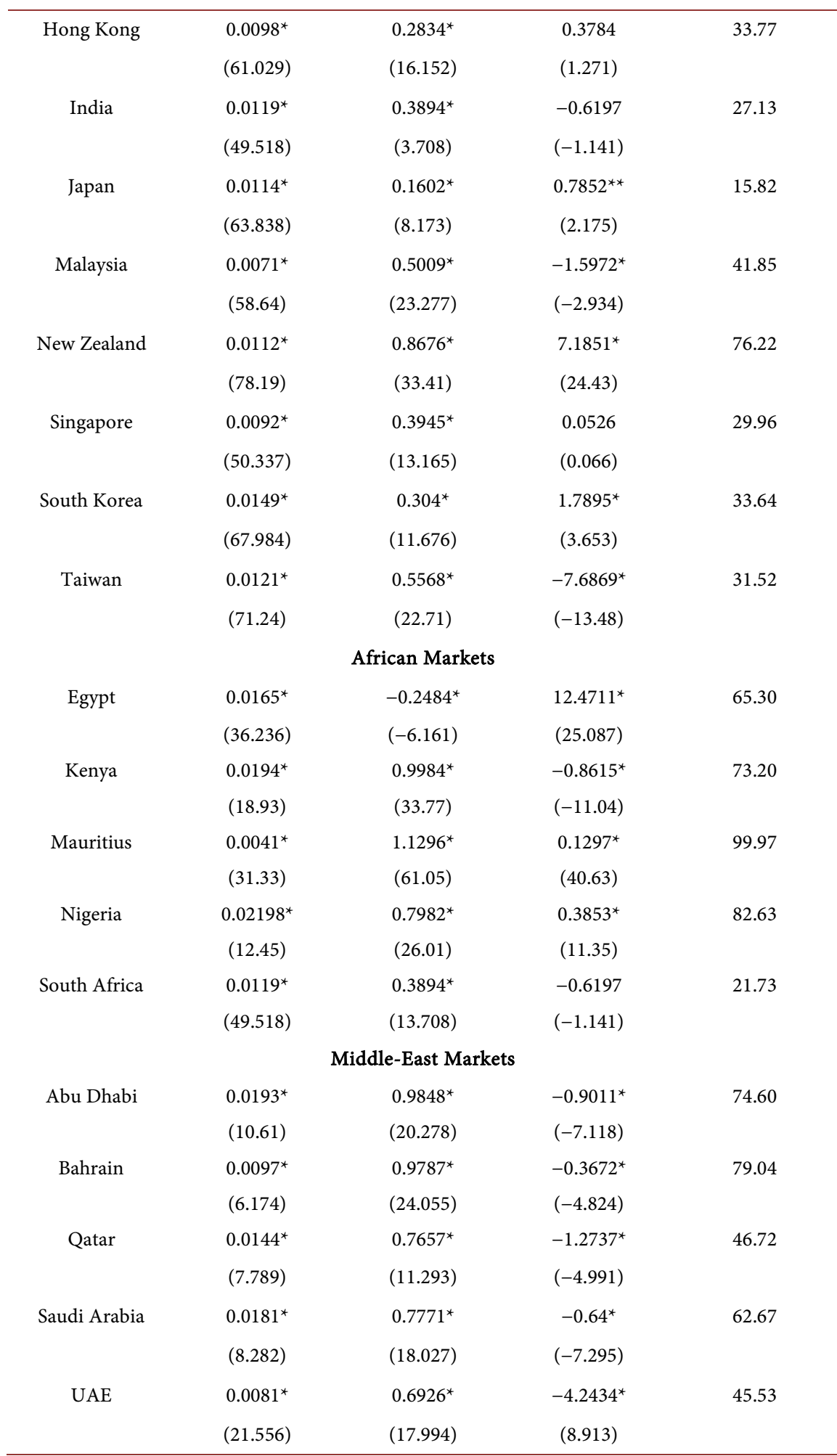

This table reports the estimated coefficients of the Equation (5)

$\left(C_{S A D}=\gamma_{0}^{-}+\gamma_{1}^{-} \cdot\left|R_{m t}\right| \cdot\right.$ Dummy $_{\text {Down }}+\gamma_{2}^{-} \cdot R_{m t}^{2} \cdot$ Dummy $\left._{\text {Down }}+\varepsilon_{t}\right)$. t-statistics are given in parentheses. A negative and significant value of $\gamma_{2}^{-}$indicates the presence of herding in declining market while a positively significant value is an indication of anti-herding. ${ }^{*}$ indicates statistical significance at $1 \%$ level. ${ }^{* *}$ indicates statistical significance at $5 \%$ level. 
Table 6. Regression estimates of herding during rising and declining market (pooled model).

\begin{tabular}{|c|c|c|c|c|c|c|c|}
\hline Country Name & $\gamma_{0}$ & $\gamma_{1}$ & $\gamma_{2}$ & $\gamma_{3}$ & Adj. $R^{2}$ & $\gamma_{2}-\gamma_{3}$ & Chi-Sq. \\
\hline \multicolumn{8}{|c|}{ American and Latin American Markets } \\
\hline \multirow[t]{2}{*}{ Brazil } & $0.0126^{*}$ & $2.119^{*}$ & $2.2231^{*}$ & 0.7091 & 34.13 & 1.514 & $54.196^{*}$ \\
\hline & $(117.76)$ & $(19.03)$ & $(11.491)$ & $(0.909)$ & & & \\
\hline \multirow[t]{2}{*}{ Canada } & $0.01223^{*}$ & $0.4074^{*}$ & $4.8383^{*}$ & $1.9393^{*}$ & 35.08 & 2.899 & $29.797^{*}$ \\
\hline & $(111.82)$ & $(20.59)$ & $(7.95)$ & $(3.37)$ & & & \\
\hline \multirow[t]{2}{*}{ Chile } & $0.0111^{*}$ & $0.1947^{*}$ & $0.7042^{*}$ & -0.1711 & 19.05 & 0.8753 & $21.265^{*}$ \\
\hline & $(97.76)$ & $(17.146)$ & $(3.731)$ & $(-0.825)$ & & & \\
\hline \multirow[t]{2}{*}{ Mexico } & $0.0091^{*}$ & $0.3961^{*}$ & $3.161^{*}$ & 0.4852 & 42.44 & 2.6758 & $49.938^{*}$ \\
\hline & (91.68) & $(26161)$ & $(8.672)$ & $(1.061)$ & & & \\
\hline \multirow[t]{2}{*}{ United States } & $0.0107^{*}$ & $0.4128^{*}$ & $0.6681^{*}$ & $-1.1264^{*}$ & 31.08 & 1.7945 & $32.050^{*}$ \\
\hline & $(61.852)$ & $(21.554)$ & $(1.891)$ & $(-2.681)$ & & & \\
\hline \multicolumn{8}{|c|}{ European Markets } \\
\hline \multirow[t]{2}{*}{ Belgium } & $0.0075^{*}$ & $0.3539^{*}$ & $2.6243^{*}$ & $1.3045^{\star}$ & 39.95 & 1.3198 & $15.309^{*}$ \\
\hline & $(66.784)$ & $(21.823)$ & (7.739) & $(2.988)$ & & & \\
\hline \multirow[t]{2}{*}{ France } & $0.0088^{*}$ & $0.2124^{*}$ & $1.6037^{\star}$ & 0.2927 & 24.6 & 1.311 & $28.684^{*}$ \\
\hline & $(75.172)$ & $(15.351)$ & $(6.031)$ & $(0.929)$ & & & \\
\hline \multirow[t]{2}{*}{ Germany } & $0.0085^{*}$ & $0.2485^{*}$ & $1.2389^{*}$ & 0.4229 & 26.45 & 0.816 & $9.8364^{*}$ \\
\hline & $(71.149)$ & $(17.918)$ & $(4.986)$ & $(1.296)$ & & & \\
\hline \multirow[t]{2}{*}{ Greece } & $0.0139^{*}$ & $0.2977^{*}$ & $1.5568^{\star}$ & -0.1089 & 97.2 & 1.6657 & $84.627^{*}$ \\
\hline & $(118.98)$ & $(34.151)$ & $(162.057)$ & $(-0.585)$ & & & \\
\hline \multirow[t]{2}{*}{ Netherland } & $0.0091^{*}$ & $0.2984^{*}$ & $3.1317^{\star}$ & $1.4574^{*}$ & 33.18 & 1.6742 & $21.800^{*}$ \\
\hline & $(56.902)$ & $(14.518)$ & $(6.955)$ & $(3.105)$ & & & \\
\hline \multirow[t]{2}{*}{ Portugal } & $0.0086^{*}$ & $0.04507^{\star}$ & $-0.9055^{\star}$ & $-2.483^{*}$ & 34.06 & 1.5775 & $34.772^{*}$ \\
\hline & $(86.054)$ & $(32.816)$ & $(-3.144)$ & $(-7.519)$ & & & \\
\hline \multirow[t]{2}{*}{ Russia } & $0.0129^{*}$ & $0.306^{*}$ & $1.1581^{\star}$ & -0.0529 & 98.39 & 1.211 & $50.716^{*}$ \\
\hline & $(110.681)$ & $(37.081)$ & $(179.288)$ & $(-0.305)$ & & & \\
\hline \multirow[t]{2}{*}{ Sweden } & $0.009^{*}$ & $0.2532^{\star}$ & $0.964^{*}$ & -0.6628 & 22.37 & 1.6268 & $34.960^{*}$ \\
\hline & $(69.738)$ & $(16.237)$ & $(3.201)$ & $(-1.82)$ & & & \\
\hline \multirow[t]{2}{*}{ Switzerland } & $0.0069^{*}$ & $0.2856^{*}$ & $2.03387^{*}$ & $0.8409^{*}$ & 36.2 & 1.19297 & $21.721^{*}$ \\
\hline & $(62.349)$ & $(20.131)$ & $(7.261)$ & $(2.607)$ & & & \\
\hline \multirow[t]{2}{*}{ United Kingdom } & $0.0095^{*}$ & $0.2426^{*}$ & $3.4359^{*}$ & $2.1526^{*}$ & 30.93 & 1.2833 & $14.231^{*}$ \\
\hline & $(86.374)$ & $(14.962)$ & $(8.671)$ & $(5.067)$ & & & \\
\hline \multicolumn{8}{|c|}{ Asian and Asia Pacific Markets } \\
\hline \multirow[t]{2}{*}{ Australia } & $0.01678^{*}$ & $0.4599^{*}$ & $10.79^{*}$ & $13.69^{*}$ & 43.35 & -2.9 & $182.960^{*}$ \\
\hline & $(185.542)$ & $(25.078)$ & $(12.801)$ & $(2.918)$ & & & \\
\hline \multirow[t]{2}{*}{ China } & $0.0118^{*}$ & $0.343^{*}$ & $-2.9947^{\star}$ & $-1.1542^{\star}$ & 23.43 & -1.8405 & $65.858^{\star}$ \\
\hline & $(82.375)$ & $(22.031)$ & $(-9.821)$ & $(-3.938)$ & & & \\
\hline \multirow[t]{2}{*}{ Hong Kong } & $0.0098^{*}$ & $0.2781^{\star}$ & $1.7914^{*}$ & -0.4654 & 33.49 & 2.2568 & $111.760^{*}$ \\
\hline & (90.692) & $(23.155)$ & $(7.699)$ & $(-1.954)$ & & & \\
\hline
\end{tabular}




\section{Continued}

\begin{tabular}{|c|c|c|c|c|c|c|c|}
\hline India & $\begin{array}{c}0.01172^{*} \\
(86.82)\end{array}$ & $\begin{array}{l}0.3796^{*} \\
(25.643)\end{array}$ & $\begin{array}{l}-0.4377 \\
(-1.714)\end{array}$ & $\begin{array}{l}-1.4157^{*} \\
(-4.717)\end{array}$ & 27.05 & 0.978 & $13.329^{*}$ \\
\hline Japan & $\begin{array}{l}0.01113^{*} \\
(97.763)\end{array}$ & $\begin{array}{l}0.1947^{\star} \\
(17.146)\end{array}$ & $\begin{array}{l}0.7043^{*} \\
(3.731)\end{array}$ & $\begin{array}{c}-1.711 \\
(-0.825)\end{array}$ & 19.05 & 2.4153 & $21.265^{\star}$ \\
\hline Malaysia & $\begin{array}{c}0.0069^{\star} \\
(100.473)\end{array}$ & $\begin{array}{l}0.4892^{*} \\
(47.783)\end{array}$ & $\begin{array}{c}0.888^{\star} \\
(120.949)\end{array}$ & $\begin{array}{l}-2.062^{\star} \\
(-6.007)\end{array}$ & 99.35 & 2.95 & $75.711^{*}$ \\
\hline New Zealand & $\begin{array}{l}0.0106^{\star} \\
(120.24)\end{array}$ & $\begin{array}{l}1.056^{\star} \\
(59.67)\end{array}$ & $\begin{array}{l}6.921^{*} \\
(29.77)\end{array}$ & $\begin{array}{c}-12.1^{\star} \\
(-13.39)\end{array}$ & 75.38 & 19.021 & $514.000^{*}$ \\
\hline Singapore & $\begin{array}{l}0.0089^{*} \\
(79.988)\end{array}$ & $\begin{array}{l}0.3977^{\star} \\
(23.547)\end{array}$ & $\begin{array}{l}1.2931^{*} \\
(3.051)\end{array}$ & $\begin{array}{l}-2.8554^{*} \\
(-4.764)\end{array}$ & 31.21 & 4.1485 & $78.764^{\star}$ \\
\hline South Korea & $\begin{array}{c}0.0154^{\star} \\
(113.641)\end{array}$ & $\begin{array}{l}0.2088^{*} \\
(14.433)\end{array}$ & $\begin{array}{l}6.6513^{*} \\
(28.154)\end{array}$ & $\begin{array}{l}1.6977^{*} \\
(6.091)\end{array}$ & 45.62 & 4.9536 & $409.630^{*}$ \\
\hline Taiwan & $\begin{array}{c}0.0126^{\star} \\
(105.557)\end{array}$ & $\begin{array}{l}0.4046^{*} \\
(23.431)\end{array}$ & $\begin{array}{l}-1.2175^{\star} \\
(-2.683)\end{array}$ & $\begin{array}{l}-3.5806^{*} \\
(-8.721)\end{array}$ & 28.48 & 2.3631 & $54.228^{*}$ \\
\hline & & & African $\mathrm{M}$ & rkets & & & \\
\hline Egypt & $\begin{array}{l}0.0147^{\star} \\
(59.479)\end{array}$ & $\begin{array}{l}0.0148^{\star} \\
(0.633)\end{array}$ & $\begin{array}{c}10.57^{*} \\
(31.295)\end{array}$ & $\begin{array}{l}2.682^{*} \\
(6.459)\end{array}$ & 45.79 & 7.888 & $487.320^{\star}$ \\
\hline Kenya & $\begin{array}{l}0.0177^{\star} \\
(30.02)\end{array}$ & $\begin{array}{l}1.0937^{\star} \\
(61.23)\end{array}$ & $\begin{array}{l}-0.9324^{*} \\
(-20.68)\end{array}$ & $\begin{array}{l}-1.7798^{*} \\
(-26.23)\end{array}$ & 73.43 & 0.8474 & $287.820^{*}$ \\
\hline Mauritius & $\begin{array}{l}0.0487^{*} \\
(61.28)\end{array}$ & $\begin{array}{l}0.9332^{*} \\
(70.85)\end{array}$ & $\begin{array}{l}0.1637^{*} \\
(71.94)\end{array}$ & $\begin{array}{l}5.002^{*} \\
(16.21)\end{array}$ & 99.95 & -4.8383 & $247.640^{*}$ \\
\hline Nigeria & $\begin{array}{l}0.0206^{*} \\
(22.42)\end{array}$ & $\begin{array}{l}0.9299^{*} \\
(46.099)\end{array}$ & $\begin{array}{l}0.1837^{\star} \\
(7.253)\end{array}$ & $\begin{array}{l}-0.9685^{*} \\
(-7.437)\end{array}$ & 76.83 & 1.1522 & $94.320^{*}$ \\
\hline South Africa & $\begin{array}{l}0.0117^{*} \\
(86.82)\end{array}$ & $\begin{array}{l}0.3795^{*} \\
(25.643)\end{array}$ & $\begin{array}{l}-0.4377 \\
(-1.714)\end{array}$ & $\begin{array}{c}-1.4157^{*} \\
(-4.717)\end{array}$ & 27.05 & 0.978 & $13.329^{*}$ \\
\hline \multicolumn{8}{|c|}{ Middle-East Markets } \\
\hline Abu Dhabi & $\begin{array}{c}0.01793^{*} \\
(22.25)\end{array}$ & $\begin{array}{l}1.0982^{*} \\
(51.05)\end{array}$ & $\begin{array}{c}-0.8697^{*} \\
(-18.23)\end{array}$ & $\begin{array}{l}-2.3047^{*} \\
(-17.96)\end{array}$ & 67.48 & 1.435 & $170.580^{*}$ \\
\hline Bahrain & $\begin{array}{l}0.0112^{*} \\
(14.97)\end{array}$ & $\begin{array}{l}0.9847^{\star} \\
(44.48)\end{array}$ & $\begin{array}{c}-0.3793^{*} \\
(-8.02)\end{array}$ & $\begin{array}{l}-2.6736^{*} \\
(-18.12)\end{array}$ & 0.27 & 2.2943 & $324.390^{*}$ \\
\hline Qatar & $\begin{array}{l}0.0141^{\star} \\
(21.557)\end{array}$ & $\begin{array}{l}0.5868^{*} \\
(41.477)\end{array}$ & $\begin{array}{l}-0.1398^{*} \\
(-5.898)\end{array}$ & $\begin{array}{l}-0.7062^{*} \\
(-12.098)\end{array}$ & 69.97 & 0.5664 & $132.240^{*}$ \\
\hline Saudi Arabia & $\begin{array}{l}0.01849^{*} \\
(23.448)\end{array}$ & $\begin{array}{l}0.6826^{\star} \\
(49.26)\end{array}$ & $\begin{array}{l}-0.1236^{*} \\
(-8.261)\end{array}$ & $\begin{array}{c}-0.9504^{*} \\
(-20.8)\end{array}$ & 68.92 & 0.8268 & $423.210^{*}$ \\
\hline UAE & $\begin{array}{l}0.0081^{*} \\
(38.174)\end{array}$ & $\begin{array}{l}0.6536^{*} \\
(29.331)\end{array}$ & $\begin{array}{l}-2.1118^{*} \\
(-6.997)\end{array}$ & $\begin{array}{l}-5.5229^{*} \\
(-14.399)\end{array}$ & 41.76 & 3.4111 & $131.510^{*}$ \\
\hline
\end{tabular}

This table reports the estimated coefficients of the Equation (6)

$\left(C S A D_{t}=\gamma_{0}+\gamma_{1} \cdot\left|R_{m t}\right|+\gamma_{2} \cdot R_{m t}^{2} \cdot\right.$ Dummy $_{U p}+\gamma_{3} \cdot R_{m t}^{2} \cdot$ Dummy $\left._{\text {Down }}+\varepsilon_{t}\right)$. t-statistics are given in parentheses. A negative and significant value of $\gamma_{2}$ indicates the presence of herding in rising market while a negatively significant value of $\gamma_{3}$ is an indication of herding in declining market. $\gamma_{2}-\gamma_{3}$ represents difference of coefficient between up and down market with respect to squared market return while chi-square value of Wald test indicates the statistical significance of the difference between two coefficients. ${ }^{*}$ indicates statistical significance at $1 \%$ level. ${ }^{*}$ indicates statistical significance at $5 \%$ level. 


$$
C S A D_{t}=\gamma_{0}+\gamma_{1} \cdot\left|R_{m t}\right|+\gamma_{2} \cdot R_{m t}^{2}+\gamma_{3} \cdot R_{m t}^{3}+\varepsilon_{t}
$$

Results of the Equation (7) are given in Table 7. Similar to the results suggested by Chiang et al. [38], herding coefficient on $R_{m t}^{2}$ become more negative in new specification in case of those countries where herding is detected using Equation (3). In these cases, a positive (negative) and significant coefficient $R_{m t}^{3}$ indicate that herding increases in a downward (upward) market. In majority of the cases adjusted $r$-square values of the model increase marginally, which indicate that new term improve the model specification.

\subsection{Quantile Regression Results}

Standard linear regression presents the mean relationship between the dependent and independent variables that may be different at the tails of the distribution. In order to obtain better picture of the empirical distribution, quantile regression would be a better approach as it provide the multiple estimates over the distribution of dependent variable [37]. In order to examine the relation of CSAD with absolute market return and its square term in various point of CSAD distribution, we apply quantile regression using Equation (3) and value of $\gamma_{2}$ over different quantile of CSAD are presented in Table 8. In case of American and Latin American markets, we infer a consistent anti-herding behaviour as reflected in repeatedly positive and significant values of $\gamma_{2}$. Picture is slightly different in case of US, where in the extreme upper part of the distribution $\left(80^{\text {th }}\right.$ and $90^{\text {th }}$ quintiles) values of $\gamma_{2}$ become negative but it is statistically insignificant. European markets are also exhibiting the same results as anti-herding indication become weak at upper quantile for majority of the markets. Germany is the only exception where at $90^{\text {th }}$ quantile $\gamma_{2}$ coefficient value turned negative and found statistically significant. Among all European markets, only Portgual is found with presence of herding and consistent results are found in case of quantile regression too. For all quantile values of $\gamma_{2}$ are negative but for some quantiles values are significant and for some these are insignificant. Results are bit different in context of Asian markets. Using Equation (3) only China, India and Taiwan exhibit herding behaviour in their stock markets but in quantile regression barring China, Hong Kong, South Korea and Taiwan, values of $\gamma_{2}$ become statistically insignificant in majority of the cases. In case of China and Taiwan values are negatively significant while in case of Hong Kong and South Korea, these are positively significant. Among African market, except for Kenya values of $\gamma_{2}$ for other countries are statistically insignificant. In all Middle-East markets herding is highly prevalent in case of the majority of cases. Similar to the results of Stavroyuannis and Babalos [37], in majority of cases no herding or anti-herding indication is seen in upper most quantile of CSAD, which may be due to non-trading of investors in case of high volatile market.

\subsection{Herding and Market Conditional Volatility}

Bensaida [23] explains that some informed investors take first move in some 
Table 7. Results of augmented model.

\begin{tabular}{|c|c|c|c|c|c|}
\hline Country Name & $\gamma_{0}$ & $\gamma_{1}$ & $\gamma_{2}$ & $\gamma_{3}$ & Adj. $R^{2}$ \\
\hline \multicolumn{6}{|c|}{ American and Latin American Markets } \\
\hline \multirow[t]{2}{*}{ Brazil } & $0.0127^{\star}$ & $0.2061^{\star}$ & $1.6305^{\star}$ & $2.8216^{\star \star}$ & 33.42 \\
\hline & $(115.976)$ & $(17.622)$ & $(7.63)$ & $(2.257)$ & \\
\hline \multirow[t]{2}{*}{ Canada } & $0.00123^{*}$ & $0.0409^{*}$ & $0.3225^{*}$ & $11.824^{*}$ & 34.83 \\
\hline & (111.609) & $(20.662)$ & $(6.094)$ & $(3.526)$ & \\
\hline \multirow[t]{2}{*}{ Chile } & $0.0011^{\star}$ & $0.1916^{\star}$ & 0.2948 & 1.1651 & 18.69 \\
\hline & $(97.239)$ & $(16.545)$ & $(1.634)$ & $(1.22)$ & \\
\hline \multirow[t]{2}{*}{ Mexico } & $0.0009^{\star}$ & $0.0413^{*}$ & $1.253^{*}$ & $18.2^{*}$ & 42.22 \\
\hline & $(88.786)$ & $(25.229)$ & $(2.984)$ & $(5.656)$ & \\
\hline \multirow[t]{2}{*}{ United States } & $0.0107^{*}$ & $0.41811^{*}$ & -0.3598 & $12.2231^{\star}$ & 31.01 \\
\hline & $(61.535)$ & $(21.578)$ & $(-0.991)$ & $(5.216)$ & \\
\hline \multicolumn{6}{|c|}{ European Markets } \\
\hline \multirow[t]{2}{*}{ Belgium } & $0.0076^{*}$ & $0.3529^{\star}$ & $1.9802^{*}$ & $6.7075^{\star \star}$ & 39.83 \\
\hline & $(66.059)$ & $(20.946)$ & $(5.201)$ & $(2.329)$ & \\
\hline \multirow[t]{2}{*}{ France } & $0.0088^{*}$ & $0.2123^{\star}$ & $0.9396^{*}$ & $6.3786^{*}$ & 24.33 \\
\hline & $(74.708)$ & $(15.048)$ & (3.419) & $(3.448)$ & \\
\hline \multirow[t]{2}{*}{ Germany } & $0.0085^{\star}$ & $0.2398^{\star}$ & $1.0449^{*}$ & 0.6761 & 26.3 \\
\hline & $(70.408)$ & $(16.425)$ & $(3.637)$ & $(0.37)$ & \\
\hline \multirow[t]{2}{*}{ Greece } & $0.0137^{\star}$ & $0.3418^{*}$ & -0.1464 & $1.5833^{\star}$ & 97.18 \\
\hline & $(102.165)$ & $(25.561)$ & $(-0.676)$ & $(8.082)$ & \\
\hline \multirow[t]{2}{*}{ Netherland } & $0.0092^{*}$ & $0.2982^{\star}$ & $2.2698^{*}$ & $9.4472^{*}$ & 32.96 \\
\hline & $(56.808)$ & $(14.454)$ & $(5.329)$ & $(3.053)$ & \\
\hline \multirow[t]{2}{*}{ Portugal } & $0.0086^{*}$ & $0.4485^{\star}$ & $-1.8963^{*}$ & $7.4844^{\star}$ & 33.72 \\
\hline & $(84.914)$ & $(31.063)$ & $(-5.792)$ & $(3.283)$ & \\
\hline \multirow[t]{2}{*}{ Russia } & $0.0128^{\star}$ & $0.3193^{\star}$ & 0.3084 & $0.5988^{\star}$ & 98.38 \\
\hline & $(95.937)$ & $(24.998)$ & $(1.561)$ & $(4.425)$ & \\
\hline \multirow[t]{2}{*}{ Sweden } & $0.009^{*}$ & $0.2536^{\star}$ & 0.1368 & $8.8478^{\star}$ & 22.05 \\
\hline & $(69.246)$ & $(15.935)$ & $(0.431)$ & (4.009) & \\
\hline \multirow[t]{2}{*}{ Switzerland } & $0.0069^{\star}$ & $0.2878^{\star}$ & $1.3549^{*}$ & $7.7503^{*}$ & 36.13 \\
\hline & $(62.068)$ & $(20.06)$ & $(4.832)$ & $(4.031)$ & \\
\hline \multirow[t]{2}{*}{ United Kingdom } & $0.0095^{*}$ & $0.2438^{\star}$ & $2.7516^{*}$ & $8.7898^{*}$ & 30.84 \\
\hline & $(86.198)$ & $(14.958)$ & $(7.291)$ & $(2.865)$ & \\
\hline \multicolumn{6}{|c|}{ Asian and Asia Pacific Markets } \\
\hline \multirow[t]{2}{*}{ Australia } & $0.01691^{*}$ & $0.4241^{*}$ & $7.659^{*}$ & $87.783^{*}$ & 42.91 \\
\hline & $(181.06)$ & $(21.22)$ & $(10.87)$ & $(12.1)$ & \\
\hline \multirow[t]{2}{*}{ China } & $0.0118^{*}$ & $0.0337^{*}$ & $-1.944^{*}$ & $-1.301^{*}$ & 23.26 \\
\hline & $(82.387)$ & $(21.63)$ & $(-7.028)$ & $(-7.471)$ & \\
\hline \multirow[t]{2}{*}{ Hong Kong } & $0.0098^{*}$ & $0.2784^{*}$ & $0.6439^{*}$ & $9.1832^{\star}$ & 34.11 \\
\hline & $(90.16)$ & $(23.017)$ & $(3.044)$ & $(7.077)$ & \\
\hline
\end{tabular}




\section{Continued}

\begin{tabular}{|c|c|c|c|c|c|}
\hline \multirow[t]{2}{*}{ India } & $0.01176^{\star}$ & $0.3749^{*}$ & $-0.8623^{*}$ & 1.4246 & 26.84 \\
\hline & $(86.004)$ & $(24.402)$ & $(-3.281)$ & $(0.984)$ & \\
\hline \multirow[t]{2}{*}{ Japan } & $0.0116^{*}$ & $0.1916^{*}$ & 0.2948 & 1.1651 & 18.69 \\
\hline & $(97.239)$ & (16.545) & $(1.634)$ & $(1.22)$ & \\
\hline \multirow[t]{2}{*}{ Malaysia } & $0.0069^{*}$ & $0.5018^{\star}$ & $-1.273^{*}$ & $1.515^{*}$ & 99.34 \\
\hline & (90.125) & $(34.457)$ & $(-3.099)$ & $(5.351)$ & \\
\hline \multirow[t]{2}{*}{ New Zealand } & $0.0108^{*}$ & $0.9577^{*}$ & $4.65^{\star}$ & $20.14^{*}$ & 72.71 \\
\hline & $(108.467)$ & $(40.863)$ & $(5.702)$ & $(3.985)$ & \\
\hline \multirow[t]{2}{*}{ Singapore } & $0.0089^{*}$ & $0.03999^{*}$ & 0.4686 & $19.9399^{*}$ & 30.59 \\
\hline & $(79.486)$ & (23.555) & $(-1.205)$ & $(6.223)$ & \\
\hline \multirow[t]{2}{*}{ South Korea } & $0.0152^{*}$ & $2576^{*}$ & $2.982^{*}$ & $29.7^{*}$ & 47.14 \\
\hline & $(112.76)$ & $(17.56)$ & (12.58) & $(23.48)$ & \\
\hline \multirow[t]{2}{*}{ Taiwan } & $0.0126^{*}$ & $0.3976^{*}$ & $-2.1444^{*}$ & $32.9803^{*}$ & 29.29 \\
\hline & $(106.371)$ & $(23.123)$ & $(-5.349)$ & $(10.307)$ & \\
\hline \multicolumn{6}{|c|}{ African Markets } \\
\hline \multirow[t]{2}{*}{ Egypt } & $0.0145^{\star}$ & $0.0803^{*}$ & $5.015^{\star}$ & $44.66^{*}$ & 48.15 \\
\hline & $(59.312)$ & $(3.444)$ & $(14.555)$ & $(25.039)$ & \\
\hline \multirow[t]{2}{*}{ Kenya } & $0.01728^{\star}$ & $1.1329^{*}$ & $-1.5051^{\star}$ & $1.0046^{*}$ & 73.62 \\
\hline & $(29.31)$ & $(61.51)$ & $(-26.98)$ & $(17.7)$ & \\
\hline \multirow[t]{2}{*}{ Mauritius } & $0.0049^{*}$ & $0.8793^{*}$ & $4.508^{*}$ & $-7.483^{\star}$ & 99.94 \\
\hline & $(57.76)$ & $(50.27)$ & $(12.56)$ & $(-12.16)$ & \\
\hline \multirow[t]{2}{*}{ Nigeria } & $0.0149^{*}$ & $1.3119^{*}$ & $-1.5742^{*}$ & $1.2132^{*}$ & 79.22 \\
\hline & $(16.28)$ & $(46.64)$ & $(-17.41)$ & $(21.11)$ & \\
\hline \multirow[t]{2}{*}{ South Africa } & $0.0118^{*}$ & $0.3749^{*}$ & $-0.8623^{\star}$ & 1.4245 & 26.84 \\
\hline & $(86.004)$ & $(24.402)$ & $(-3.281)$ & $(0.984)$ & \\
\hline \multicolumn{6}{|c|}{ Middle-East Markets } \\
\hline \multirow[t]{2}{*}{ Abu Dhabi } & $0.0155^{\star}$ & $1.3042^{\star}$ & $-2.4523^{*}$ & $1.7769^{\star}$ & 67.69 \\
\hline & $(18.41)$ & $(42.42)$ & $(-18.34)$ & $(13.63)$ & \\
\hline \multirow[t]{2}{*}{ Bahrain } & $0.0096^{*}$ & $1.1004^{*}$ & $-1.777^{*}$ & $1.8595^{\star}$ & 67.88 \\
\hline & $(11.67)$ & $(33.88)$ & $(-11.9)$ & $(11.51)$ & \\
\hline \multirow[t]{2}{*}{ Qatar } & $0.01198^{\star}$ & $0.7172^{\star}$ & $-0.8461^{\star}$ & $0.6142^{*}$ & 71.51 \\
\hline & $(18.15)$ & $(40.35)$ & $(-15.85)$ & (15.63) & \\
\hline \multirow[t]{2}{*}{ Saudi Arabia } & $0.0153^{*}$ & $0.8519^{*}$ & $-0.9578^{*}$ & $0.4685^{\star}$ & 69.15 \\
\hline & $(18.65)$ & $(45.16)$ & $(-21.23)$ & $(21.03)$ & \\
\hline \multirow[t]{2}{*}{ UAE } & $0.0081^{*}$ & $0.6571^{*}$ & $-3.972^{\star}$ & $13.5548^{*}$ & 40.14 \\
\hline & $(37.375)$ & $(28.106)$ & $(-11.71)$ & $(7.892)$ & \\
\hline
\end{tabular}

This table reports the estimated coefficients of the Equation (7)

$\left(C S A D_{t}=\gamma_{0}+\gamma_{1} \cdot\left|R_{m t}\right|+\gamma_{2} \cdot R_{m t}^{2}+\gamma_{3} \cdot R_{m t}^{3}+\varepsilon_{t}\right) . \mathrm{t}$-statistics are given in parentheses. A negative and significant value of $\gamma_{2}$ indicates the presence of herding in market while a positively significant value is an indication of anti-herding. $\gamma_{3}$ is showing the impact of interaction variable of $R_{m t}$ and $R_{m t}^{2}$. A positive (negative) and significant coefficient $R_{m t}^{3}$ indicate that herding increases in a downward (upward) market. ${ }^{*}$ indicates statistical significance at $1 \%$ level. ${ }^{*}$ indicates statistical significance at $5 \%$ level. 
Table 8. Estimates of $\gamma_{2}$ over different quantile of CSAD.

\begin{tabular}{|c|c|c|c|c|c|c|c|c|c|}
\hline Quantiles $\rightarrow$ & 10 & 20 & 30 & 40 & 50 & 60 & 70 & 80 & 90 \\
\hline \multicolumn{10}{|c|}{ American and Latin American Markets } \\
\hline \multirow[t]{2}{*}{ Brazil } & $1.6755^{\star}$ & $1.3803^{*}$ & $1.55236^{*}$ & $2.1941^{*}$ & $2.3768^{\star}$ & $2.5106^{\star}$ & $2.1847^{\star}$ & $3.4658^{\star *}$ & $5.8224^{* *}$ \\
\hline & $(4.2517)$ & $(7.5943)$ & $(4.5358)$ & $(2.6498)$ & $(3.8868)$ & $(8.3227)$ & $(4.6526)$ & $(2.3828)$ & $(2.5521)$ \\
\hline \multirow[t]{2}{*}{ Canada } & $2.1369^{* *}$ & 3.5885 & $5.5738^{\star}$ & $4.9025^{\star}$ & $5.2993^{\star}$ & $5.3648^{\star}$ & $5.1871^{*}$ & $5.692^{\star}$ & 5.1083 \\
\hline & $(2.292)$ & $(1.9955)$ & $(7.3989)$ & $(23.8641)$ & $(3.2544)$ & $(3.6242)$ & $(3.009)$ & $(2.9725)$ & $(1.1138)$ \\
\hline \multirow[t]{2}{*}{ Chile } & $0.7386^{\star}$ & 0.5168 & 0.3694 & 0.2096 & 0.1835 & 0.1912 & 0.7219 & 1.1647 & 1.1831 \\
\hline & $(2.7786)$ & $(1.3768)$ & $(0.8861)$ & $(0.6007)$ & $(0.8436)$ & $(0.2279)$ & $(0.9782)$ & $(1.8131)$ & $(1.601)$ \\
\hline \multirow{2}{*}{ Mexico } & 0.0498 & 1.3406 & $3.2141^{\star *}$ & $3.2681^{\star *}$ & $3.063^{*}$ & $3.2652^{\star}$ & $2.8999^{*}$ & 2.4845 & 5.0885 \\
\hline & $(0.0733)$ & $(0.6089)$ & $(2.4258)$ & $(2.5458)$ & $(3.2372)$ & $(2.9683)$ & $(2.6408)$ & $(1.1193)$ & $(1.1744)$ \\
\hline \multirow[t]{2}{*}{ United States } & $1.2813^{*}$ & $1.7189^{*}$ & $2.0473^{*}$ & $1.776^{*}$ & 2.4728 & $3.4397^{\star}$ & $2.4417^{\star}$ & -0.0334 & -0.2417 \\
\hline & $(3.1947)$ & $(4.1316)$ & $(3.9696)$ & $(2.6152)$ & $(1.8728)$ & $(2.7356)$ & $(4.1895)$ & $(-0.0501)$ & $(-0.1578)$ \\
\hline \multicolumn{10}{|c|}{ European Markets } \\
\hline \multirow[t]{2}{*}{ Belgium } & $3.2127^{\star}$ & $3.2224^{\star}$ & $2.6979^{*}$ & $2.5847^{\star \star}$ & $3.7068^{\star}$ & $3.4114^{*}$ & $3.0164^{* *}$ & $2.9794^{\star}$ & 3.4128 \\
\hline & $(8.8699)$ & (11.6089) & $(5.4162)$ & $(2.1317)$ & $(3.1877)$ & $(12.0081)$ & $(2.5016)$ & $(2.609)$ & $(0.7927)$ \\
\hline \multirow[t]{2}{*}{ France } & $2.2096^{*}$ & $2.0932^{*}$ & $1.9489^{*}$ & $2.0822^{*}$ & $1.5486^{*}$ & $1.3431^{* *}$ & $1.0695^{\star}$ & 0.2506 & -0.6980 \\
\hline & $(6.658)$ & $(5.6273)$ & $(5.0462)$ & $(5.8811)$ & $(3.1784)$ & $(2.2661)$ & $(3.4824)$ & $(0.50302)$ & $(-0.9599)$ \\
\hline \multirow[t]{2}{*}{ Germany } & $1.1819^{*}$ & $1.8136^{* *}$ & $2.2183^{*}$ & $2.311^{\star}$ & $1.8966^{\star}$ & $2.1286^{\star *}$ & 1.5277 & 0.6859 & $-0.5584^{*}$ \\
\hline & $(2.9168)$ & $(2.4415)$ & $(3.4168)$ & $(6.1936)$ & $(6.1492)$ & $(2.2893)$ & (1.9159) & $(0.7431)$ & $(-2.0634)$ \\
\hline \multirow[t]{2}{*}{ Greece } & 1.7327 & 1.6995 & 1.6604 & 1.6339 & 1.6055 & 1.5616 & 1.5152 & 1.4718 & 1.4304 \\
\hline & $(0.1243)$ & $(0.0804)$ & $(0.0743)$ & $(0.0916)$ & $(0.0664)$ & $(0.0598)$ & $(0.0525)$ & $(0.05516)$ & $(0.0402)$ \\
\hline \multirow[t]{2}{*}{ Netherland } & $3.43855^{\star}$ & $2.9182^{\star}$ & $2.8016^{\star}$ & $2.6561^{\star}$ & $2.7565^{\star}$ & $2.7934^{\star}$ & $2.6361^{\star}$ & $2.7832^{\star *}$ & 1.5215 \\
\hline & (3.5583) & (4.9967) & $(4.6879)$ & (3.8403) & (3.2096) & $(2.7192)$ & (3.1497) & $(2.4207)$ & $(1.3346)$ \\
\hline \multirow[t]{2}{*}{ Portugal } & $-2.6768^{* *}$ & -2.0759 & $-0.9656^{*}$ & -1.4326 & -1.0988 & -1.3301 & -1.5400 & -1.7598 & $-1.7865^{\star}$ \\
\hline & $(-2.3901)$ & $(-1.5606)$ & $(-3.568)$ & $(-1.653)$ & $(-1.9231)$ & $(-1.7517)$ & $(-1.9735)$ & $(-1.413)$ & $(-4.937)$ \\
\hline \multirow[t]{2}{*}{ Russia } & 1.2727 & 1.2481 & 1.2243 & 1.2004 & 1.1793 & 1.1562 & 1.0139 & 1.1138 & 1.0789 \\
\hline & $(0.4459)$ & $(0.0453)$ & $(0.0412)$ & $(0.0348)$ & $(0.03319)$ & $(0.0403)$ & $(0.03114)$ & $(0.0204)$ & $(0.03116)$ \\
\hline \multirow[t]{2}{*}{ Sweden } & $1.2518^{\star}$ & $1.2771^{\star}$ & $1.2448^{\star \star}$ & $1.3749^{*}$ & $1.2508^{* *}$ & 2.3526 & 1.2352 & 1.5260 & 2.3540 \\
\hline & $(5.5845)$ & $(2.9281)$ & $(2.1207)$ & $(7.8608)$ & $(2.052)$ & $(0.6958)$ & $(0.4867)$ & $(0.3518)$ & $(0.3683)$ \\
\hline \multirow[t]{2}{*}{ Switzerland } & $2.7466^{*}$ & $2.5785^{\star}$ & $3.4734^{\star}$ & $3.3441^{*}$ & $2.9842^{\star}$ & $2.4372^{\star}$ & $1.8131^{\star}$ & 0.8061 & 0.4012 \\
\hline & $(3.5296)$ & $(3.3019)$ & $(7.1853)$ & $(6.25)$ & (7.5494) & $(4.48864)$ & $(3.0751)$ & (1.4081) & $(0.2189)$ \\
\hline \multirow[t]{2}{*}{ United Kingdom } & $2.9971^{\star}$ & $3.4376^{\star}$ & $3.5191^{\star}$ & $4.6114^{*}$ & $4.2041^{\star}$ & $4.2256^{\star}$ & $3.4957^{\star}$ & $2.4076^{\star *}$ & 2.8171 \\
\hline & $(13.1401)$ & $(5.0813)$ & $(3.0982)$ & $(6.3472)$ & $(19.4076)$ & $(5.0676)$ & $(5.2763)$ & $(2.2773)$ & $(1.2063)$ \\
\hline \multicolumn{10}{|c|}{ Asian and Asia Pacific Markets } \\
\hline \multirow[t]{2}{*}{ Australia } & 0.7910 & 0.1943 & 3.5059 & 5.0443 & 5.2708 & 6.4115 & $7.5854^{\star}$ & $7.8042^{*}$ & $7.4019^{* *}$ \\
\hline & $(0.4386)$ & $(0.4647)$ & $(1.3768)$ & $(1.6645)$ & $(1.7618)$ & $(1.6137)$ & $(5.123)$ & $(3.68)$ & $(2.2746)$ \\
\hline \multirow[t]{2}{*}{ China } & $-1.2865^{\star}$ & $-1.1709^{*}$ & $-1.2691^{*}$ & $-1.1951^{*}$ & $-1.3226^{*}$ & $-1.4452^{*}$ & $-1.4724^{*}$ & $-1.5614^{*}$ & -1.4267 \\
\hline & $(-4.4874)$ & $(-3.6413)$ & $(-3.6412)$ & $(-3.17764)$ & $(-6.6296)$ & $(-2.4203)$ & $(-6.2791)$ & $(-3.7731)$ & $(-1.7708)$ \\
\hline
\end{tabular}




\section{Continued}

\begin{tabular}{|c|c|c|c|c|c|c|c|c|c|}
\hline \multirow[t]{2}{*}{ Hong Kong } & $1.4449^{*}$ & $1.2456^{\star *}$ & $1.0921^{\star}$ & $1.5537^{\star}$ & $1.2874^{\star *}$ & $1.9677^{\star *}$ & 1.3429 & $1.2396^{*}$ & 0.9449 \\
\hline & $(4.0706)$ & $(2.0429)$ & $(2.8638)$ & $(2.6764)$ & $(2.5107)$ & $(2.2932)$ & $(1.5341)$ & $(4.0323)$ & $(0.5338)$ \\
\hline \multirow[t]{2}{*}{ India } & 0.2728 & 0.3838 & 0.3634 & 0.1036 & -0.2524 & -0.1708 & -0.1697 & -0.9638 & -0.9165 \\
\hline & $(0.6745)$ & $(0.5447)$ & $(0.6789)$ & $(0.5315)$ & $(-0.9933)$ & $(-0.1891)$ & $(-0.6848)$ & $(-0.1014)$ & $(-0.5089)$ \\
\hline \multirow[t]{2}{*}{ Japan } & $0.7386^{*}$ & 0.5168 & 0.3694 & 0.2096 & 0.1835 & 0.1912 & 0.7219 & 1.6147 & 1.8131 \\
\hline & $(2.7786)$ & $(1.3768)$ & $(0.8861)$ & $(0.6007)$ & $(0.8436)$ & $(0.2279)$ & $(0.9783)$ & $(1.1831)$ & $(1.6011)$ \\
\hline \multirow[t]{2}{*}{ Malaysia } & 0.9849 & 0.9616 & 0.9392 & 0.9326 & 0.9211 & 0.9019 & 0.8893 & 0.8829 & 0.8512 \\
\hline & $(0.0029)$ & $(0.0038)$ & $(0.0036)$ & $(0.0064)$ & $(0.0033)$ & $(0.0033)$ & $(0.0033)$ & $(0.0026)$ & $(0.0029)$ \\
\hline \multirow[t]{2}{*}{ New Zealand } & -0.3757 & 8.8986 & $8.079^{\star}$ & $7.5574^{\star}$ & 7.1582 & $10.3125^{\star}$ & 15.1337 & $17.1774^{*}$ & 18.3041 \\
\hline & $(-0.1128)$ & $(0.7631)$ & $(3.023)$ & (2.959) & $(0.9391)$ & $(6.7348)$ & $(1.1904)$ & $(3.1748)$ & $(1.674)$ \\
\hline \multirow[t]{2}{*}{ Singapore } & 1.0248 & 0.2626 & 0.0607 & 0.6775 & 0.2642 & -0.1115 & -0.3707 & -1.2249 & -1.2395 \\
\hline & $(1.9812)$ & $(0.8724)$ & $(0.0655)$ & $(0.9503)$ & $(0.5382)$ & $(-0.1596)$ & $(-0.5077)$ & $(-0.8692)$ & $(-1.0374)$ \\
\hline \multirow[t]{2}{*}{ South Korea } & 0.4884 & $1.5084^{* *}$ & $1.6893^{* *}$ & $1.6895^{\star *}$ & $1.3955^{\star}$ & $2.3922^{\star}$ & 2.5129 & 4.5839 & $9.6837^{*}$ \\
\hline & $(0.3125)$ & $(2.1436)$ & $(2.4747)$ & $(2.2868)$ & $(2.8965)$ & $(2.9175)$ & $(1.8844)$ & $(1.3891)$ & $(10.4183)$ \\
\hline \multirow[t]{2}{*}{ Taiwan } & $-5.3118^{*}$ & $-5.7862^{*}$ & $-5.7441^{*}$ & $-6.0844^{*}$ & $-6.5666^{*}$ & $-7.0905^{\star}$ & $-6.6997^{\star}$ & $-7.2284^{*}$ & $-5.2461^{*}$ \\
\hline & $(-6.7525)$ & $(-9.5049)$ & $(-14.1762)$ & $(-27.6544)$ & $(-30.3809)$ & $(-12.6707)$ & $(-9.5614)$ & $(-6.5862)$ & $(-2.3752)$ \\
\hline \multicolumn{10}{|c|}{ African Markets } \\
\hline \multirow[t]{2}{*}{ Egypt } & -1.8898 & -0.2910 & 0.0801 & $-0.0838^{\star}$ & -0.1736 & 0.1312 & 1.6385 & 5.1810 & $11.4176^{* *}$ \\
\hline & $(-1.0821)$ & $(-0.3097)$ & $(0.1564)$ & $(-2.045)$ & $(-0.1829)$ & $(-0.1753)$ & $(0.5188)$ & $(0.7926)$ & $(2.4869)$ \\
\hline \multirow[t]{2}{*}{ Kenya } & $-6.4427^{\star}$ & $-0.6496^{\star}$ & $-0.7065^{\star}$ & $-0.8203^{*}$ & $-0.9042^{\star}$ & $-1.1303^{*}$ & $-1.3361^{\star}$ & $-1.6577^{\star}$ & $-2.2854^{*}$ \\
\hline & $(-3.851)$ & $(-4.3335)$ & $(-5.9223)$ & $(-9.8069)$ & $(-12.6976)$ & $(-13.7824)$ & $(-9.6387)$ & $(-8.3219)$ & $(-6.715)$ \\
\hline \multirow[t]{2}{*}{ Mauritius } & 0.1907 & 0.1782 & 0.1659 & 0.1570 & 0.1493 & 0.1412 & 0.1350 & 0.1239 & 0.1047 \\
\hline & $(0.000)$ & $(0.000)$ & $(0.000)$ & $(0.000)$ & $(0.000)$ & $(0.000)$ & $(0.000)$ & $(0.000)$ & $(0.000)$ \\
\hline \multirow[t]{2}{*}{ Nigeria } & 0.0555 & -0.0621 & $-0.0595^{\star *}$ & -0.1741 & $-0.2916^{*}$ & -0.3976 & -0.0182 & 0.2548 & -0.0515 \\
\hline & $(0.2205)$ & $(-0.4311)$ & $(-2.2725)$ & $(-1.4958)$ & $(-3.328)$ & $(-0.845)$ & $(-0.8328)$ & $(0.8928)$ & $(-0.0389)$ \\
\hline \multirow[t]{2}{*}{ South Africa } & 0.2728 & 0.3838 & 0.3634 & 0.1036 & -0.2524 & -0.1708 & -0.1697 & -0.9639 & -0.9165 \\
\hline & $(0.6745)$ & $(0.5447)$ & $(0.6789)$ & $(0.5315)$ & $(-0.9933)$ & $(-0.189)$ & $(-0.6848)$ & $(-1.1014)$ & $(-0.5089)$ \\
\hline \multicolumn{10}{|c|}{ Middle-East Markets } \\
\hline \multirow[t]{2}{*}{ Abu Dhabi } & $-0.8722^{\star *}$ & $-1.1428^{\star *}$ & -0.6588 & $-0.7975^{\star *}$ & $-0.7562^{\star *}$ & $-0.8866^{\star}$ & $-1.1303^{\star}$ & $-1.3453^{\star}$ & $-1.1975^{\star}$ \\
\hline & $(-2.1985)$ & $(-2.1973)$ & $(-1.6937)$ & $(-2.2231)$ & $(-2.4735)$ & $(-22.0616)$ & $(-2.9982)$ & $(-2.9773)$ & $(-3.5261)$ \\
\hline \multirow[t]{2}{*}{ Bahrain } & -0.1242 & -0.0298 & -0.2018 & -0.3619 & -0.3615 & $-0.5197^{\star}$ & $-0.6413^{* *}$ & -0.5532 & -1.1138 \\
\hline & $(-1.4703)$ & $(-0.2821)$ & $(-1.5159)$ & $(-1.501)$ & $(-1.3988)$ & $(-3.1917)$ & $(-2.3592)$ & $(-0.9203)$ & $(-1.324)$ \\
\hline \multirow[t]{2}{*}{ Qatar } & 0.0165 & -0.1809 & $-0.2486^{*}$ & $-0.3574^{*}$ & $-0.4859^{*}$ & $-0.5457^{\star \star}$ & $-0.5033^{*}$ & $-0.5418^{\star}$ & -0.2777 \\
\hline & $(-0.6536)$ & $(-1.4985)$ & $(-7.8385)$ & $(-5.5613)$ & $(-5.0249)$ & $(-2.2788)$ & $(-2.7993)$ & $(-2.9497)$ & $(-1.6515)$ \\
\hline \multirow[t]{2}{*}{ Saudi Arabia } & 0.3485 & $-0.4924^{*}$ & -0.2932 & $-0.2903^{\star *}$ & $-0.3915^{\star}$ & -0.3908 & -0.3862 & -0.2458 & -0.2634 \\
\hline & $(-1.4039)$ & $(-4.3222)$ & $(-0.6179)$ & $(-2.2745)$ & $(-5.2422)$ & $(-1.2743)$ & $(-1.1989)$ & $(-1.3934)$ & $(-0.346)$ \\
\hline \multirow[t]{2}{*}{ UAE } & $-5.6702^{\star}$ & $-4.6476^{\star}$ & $-3.1788^{\star}$ & $-3.3686^{*}$ & $-2.8944^{*}$ & $-3.3704^{\star}$ & $-3.8241^{\star}$ & $-4.2533^{\star}$ & -4.5847 \\
\hline & $(-12.8023)$ & $(-3.2809)$ & $(-3.7026)$ & $(-4.5791)$ & $(-3.5879)$ & $(-10.6924)$ & $(-7.5734)$ & $(-6.0877)$ & $(-1.4798)$ \\
\hline
\end{tabular}

This table presents the values of $\gamma_{2}$ over different quantile of CSAD calculated using Equation (3) $\left(\operatorname{CSAD}_{t}=\gamma_{0}+\gamma_{1} \cdot\left|R_{m t}\right|+\gamma_{2} \cdot R_{m t}^{2}+\varepsilon_{t}\right)$ through quantile regression approach. t-statistics are given in parentheses. A negatively significant value of indicate the presence of herding while positively significant highlight the anti-herding behaviour in that market. ${ }^{*}$ indicates statistical significance at $1 \%$ level. ${ }^{* *}$ indicates statistical significance at $5 \%$ level. 
stocks and thereafter followed by other investors as a results trading volume of those specific stocks about which information were available face abnormally high trading volume resulted in high volatility in these stocks. If the market is enough large, overall volatility comes down [41]. Thus herding behaviour positively affects the volatility of some specific share while negatively affect the overall stock market volatility [42]. Pochea et al. [1] also argue about the investors' tendency to herd is more persistent during period characterized by increased volatility. Therefore, it will be interesting to examine asymmetric relationship of herding behaviour with market volatility using dummy variable regression presented in Equation (8). As per the suggestions of Kabir and Shakur [34] and Bensaida [23], we calculate the conditional volatility of the stock market using asymmetric GJR-GARCH $(1,1)$ model.

$$
\begin{aligned}
C S A D_{t}= & \gamma_{0}+\gamma_{1} \cdot\left|R_{m t}\right| \cdot D_{V o l}+\gamma_{2} \cdot\left|R_{m t}\right| \cdot\left(1-D_{V o l}\right)+\gamma_{3} \cdot R_{m t}^{2} \cdot D_{V o l} \\
& +\gamma_{4} \cdot R_{m t}^{2} \cdot\left(1-D_{V o l}\right)+\varepsilon_{t}
\end{aligned}
$$

where, $D_{V o l}$ is 1 when conditional volatility of market is higher than the average conditional volatility of previous 30 days else 0 . Chaing and Zheng [26] state that it is worthwhile to study the impact of exogenous variable by multiplying the absolute market return and its square term by $(1-D)$. It will help to clearly identify their effect during low volatility period also. $D_{V o l}$ represents the days of high volatility while $\left(1-D_{V o l}\right)$ represents the days of low volatility. Hence, we also multiply both the terms with $(1-D)$ and the results of the whole regression is presented in Table 9. Value of $\gamma_{3}$ and $\gamma_{4}$ indicate the presence or absence of herding during high and low market volatility regime, respectively.

In majority of cases, results are same under high volatility and low volatility regime. Herding behaviour is observed in Portugal, India, South Africa and UAE during high market volatility. In low volatility regime herding coefficient turned positive in case of Portugal while it remain negative in case of India, South Africa and UAE but in all cases values are statistically insignificant which indicates the absence of herding in these markets during low volatility. Another interesting result is found in case of Malaysia, Taiwan, Nigeria and Bahrain where high herding is observed during high volatility while during low volatility these same markets are showing anti-herding behaviour. Explanation of the same can be find in behavioural science, which explains the human tendency to feel comfortable to be part of herd during period of abnormal information flow, loss and volatility as they seek conformity of their action [27].

\subsection{Herding and Market Trading Volume}

Rising volume attract more informed investors and uninformed investors tend to mimic them as a result it formulate a herd [19] [31]. On the other side, low trading volume also promote herding as it prompt the investors to focus only on those stock which are in trade and having sufficient volume. Therefore, role of the trading volume on herding become crucial to study. We employ the model 
Table 9. Regression estimates of herding behaviour on days of high and low volatility.

\begin{tabular}{|c|c|c|c|c|c|c|}
\hline Country Name & $\gamma_{0}$ & $\gamma_{1}$ & $\gamma_{2}$ & $\gamma_{3}$ & $\gamma_{4}$ & Adj. $R^{2}$ \\
\hline \multicolumn{7}{|c|}{ American and Latin American Markets } \\
\hline \multirow[t]{2}{*}{ Brazil } & $0.0142^{*}$ & $0.2092^{*}$ & 0.0251 & $1.2669^{*}$ & $6.8451^{\star}$ & 36.29 \\
\hline & $(77.176)$ & $(11.873)$ & $(0.927)$ & $(5.12)$ & $(9.493)$ & \\
\hline \multirow[t]{2}{*}{ Canada } & $0.0122^{*}$ & $0.4122^{*}$ & $0.3731^{*}$ & $2.3111^{*}$ & 5.6969 & 35.93 \\
\hline & $(112.174)$ & $(18.774)$ & $(14.973)$ & $(3.871)$ & $(0.949)$ & \\
\hline \multirow[t]{2}{*}{ Chile } & $0.0112^{\star}$ & $0.2079^{*}$ & $0.1313^{*}$ & 0.1171 & $1.663^{*}$ & 20.03 \\
\hline & $(95.309)$ & $(16.858)$ & $(6.919)$ & $(0.66)$ & $(3.022)$ & \\
\hline \multirow[t]{2}{*}{ Mexico } & $0.0093^{\star}$ & $0.4137^{\star}$ & $0.3401^{*}$ & $2.1831^{*}$ & $2.6318^{\star}$ & 42.5 \\
\hline & $(84.059)$ & $(23.935)$ & $(14.33)$ & $(5.617)$ & $(3.292)$ & \\
\hline \multirow[t]{2}{*}{ United States } & $0.0107^{\star}$ & $0.4034^{*}$ & $0.3989^{*}$ & 0.4878 & -0.6251 & 31.06 \\
\hline & $(62.161)$ & $(18.93)$ & $(16.318)$ & $(1.263)$ & $(-1.116)$ & \\
\hline \multicolumn{7}{|c|}{ European Markets } \\
\hline \multirow[t]{2}{*}{ Belgium } & $0.0077^{\star}$ & $0.3319^{*}$ & $0.2852^{*}$ & $2.1959^{*}$ & $5.1504^{\star}$ & 40.05 \\
\hline & $(65.837)$ & $(18.969)$ & $(11.85)$ & $(6.143)$ & $(6.885)$ & \\
\hline \multirow[t]{2}{*}{ France } & $0.0088^{*}$ & $0.2039^{*}$ & $0.1821^{*}$ & $1.0796^{*}$ & $1.9116^{*}$ & 24.92 \\
\hline & $(75.597)$ & $(13.571)$ & $(10.264)$ & $(3.745)$ & $(4.603)$ & \\
\hline \multirow[t]{2}{*}{ Germany } & $0.0106^{*}$ & $0.3945^{*}$ & $0.3618^{*}$ & -0.3642 & $1.5206^{*}$ & 27.51 \\
\hline & $(49.062)$ & $(16.229)$ & $(15.251)$ & $(-0.898)$ & $(3.731)$ & \\
\hline \multirow[t]{2}{*}{ Greece } & $0.0145^{\star}$ & $0.2469^{*}$ & $0.1941^{*}$ & $1.6042^{*}$ & $3.0071^{*}$ & 97.17 \\
\hline & (114.517) & $(30.632)$ & $(10.922)$ & $(175.669)$ & $(7.546)$ & \\
\hline \multirow[t]{2}{*}{ Netherland } & $0.0093^{*}$ & $0.2755^{*}$ & $0.2017^{\star}$ & $2.1015^{*}$ & $6.8435^{\star}$ & 34.35 \\
\hline & $(57.014)$ & $(12.145)$ & $(6.957)$ & $(4.63)$ & $(8.02)$ & \\
\hline \multirow[t]{2}{*}{ Portugal } & $0.0088^{*}$ & $0.4253^{*}$ & $0.3687^{*}$ & $-1.3814^{*}$ & 1.6034 & 33.57 \\
\hline & $(81.238)$ & $(28.331)$ & $(13.832)$ & $(-4.736)$ & $(1.413)$ & \\
\hline \multirow[t]{2}{*}{ Russia } & $0.0131^{\star}$ & $0.2996^{*}$ & $0.2709^{*}$ & $0.6348^{* *}$ & $1.1829^{*}$ & 98.48 \\
\hline & $(104.287)$ & $(18.039)$ & $(32.022)$ & $(2.068)$ & $(178.964)$ & \\
\hline \multirow[t]{2}{*}{ Sweden } & $0.0091^{\star}$ & $0.2418^{\star}$ & $0.2167^{\star}$ & 0.4779 & $1.0151^{\star *}$ & 22.17 \\
\hline & $(71.085)$ & $(13.911)$ & $(11.655)$ & $(1.37)$ & $(2.424)$ & \\
\hline \multirow[t]{2}{*}{ Switzerland } & $0.0069^{*}$ & $0.2682^{\star}$ & $0.2599^{*}$ & $1.4647^{\star}$ & $2.8788^{\star}$ & 36.45 \\
\hline & $(62.013)$ & $(17.273)$ & $(13.662)$ & $(4.919)$ & $(6.141)$ & \\
\hline \multirow[t]{2}{*}{ United Kingdom } & $0.0094^{*}$ & $0.2186^{\star}$ & $0.2549^{*}$ & $3.1486^{*}$ & $3.1295^{\star}$ & 32 \\
\hline & $(86.463)$ & $(12.343)$ & $(12.374)$ & $(7.541)$ & $(5.373)$ & \\
\hline \multicolumn{7}{|c|}{ Asian and Asia Pacific Markets } \\
\hline \multirow[t]{2}{*}{ Australia } & $0.0166^{*}$ & $0.4838^{*}$ & $0.6573^{*}$ & 2.0791 & $9.373^{*}$ & 44 \\
\hline & $(179.157)$ & $(27.32)$ & $(17.875)$ & $(1.409)$ & $(4.555)$ & \\
\hline \multirow[t]{2}{*}{ China } & $0.0119^{*}$ & $0.393^{*}$ & $0.2765^{*}$ & $-2.6569^{*}$ & $-0.9732^{*}$ & 23.6 \\
\hline & $(82.835)$ & $(21.425)$ & $(15.002)$ & $(-7.626)$ & $(-2.62)$ & \\
\hline \multirow[t]{2}{*}{ Hong Kong } & $0.0099^{*}$ & $0.2619^{*}$ & $0.2344^{\star}$ & $0.6784^{\star}$ & $2.4184^{*}$ & 34.21 \\
\hline & $(88.673)$ & (19.113) & (12.996) & $(3.025)$ & $(4.826)$ & \\
\hline
\end{tabular}




\section{Continued}

\begin{tabular}{|c|c|c|c|c|c|c|}
\hline India & $\begin{array}{l}0.0117^{*} \\
(89.441)\end{array}$ & $\begin{array}{l}0.3971^{*} \\
(22.551)\end{array}$ & $\begin{array}{l}0.3291^{*} \\
(19.739)\end{array}$ & $\begin{array}{l}-1.1277^{*} \\
(-3.345)\end{array}$ & $\begin{array}{l}-0.4442 \\
(-1.585)\end{array}$ & 28.42 \\
\hline Japan & $\begin{array}{l}0.0112^{*} \\
(95.309)\end{array}$ & $\begin{array}{l}0.2079^{\star} \\
(16.858)\end{array}$ & $\begin{array}{l}0.1313^{*} \\
(6.919)\end{array}$ & $\begin{array}{l}0.1171 \\
(0.66)\end{array}$ & $\begin{array}{l}1.6631^{*} \\
(3.022)\end{array}$ & 20.03 \\
\hline Malaysia & $\begin{array}{l}0.0071^{*} \\
(99.934)\end{array}$ & $\begin{array}{l}0.5075^{\star} \\
(27.588)\end{array}$ & $\begin{array}{l}0.4256^{*} \\
(38.082)\end{array}$ & $\begin{array}{l}-1.893^{*} \\
(-2.881)\end{array}$ & $\begin{array}{c}0.9335^{\star} \\
(116.804)\end{array}$ & 99.37 \\
\hline New Zealand & $\begin{array}{l}0.0112^{*} \\
(118.6)\end{array}$ & $\begin{array}{l}0.8178^{*} \\
(35.58)\end{array}$ & $\begin{array}{l}0.9098^{*} \\
(43.58)\end{array}$ & $\begin{array}{l}10.22^{*} \\
(19.72)\end{array}$ & $\begin{array}{l}7.185^{*} \\
(27.38)\end{array}$ & 72.9 \\
\hline Singapore & $\begin{array}{l}0.0091^{*} \\
(77.924)\end{array}$ & $\begin{array}{l}0.3909^{\star} \\
(20.056)\end{array}$ & $\begin{array}{l}0.3671^{\star} \\
(15.846)\end{array}$ & $\begin{array}{l}-0.5456 \\
(-1.271)\end{array}$ & $\begin{array}{l}1.3882 \\
(1.834)\end{array}$ & 30.43 \\
\hline South Korea & $\begin{array}{c}0.0154^{*} \\
(119.158)\end{array}$ & $\begin{array}{l}0.3182^{*} \\
(18.892)\end{array}$ & $\begin{array}{l}0.0819^{*} \\
(5.047)\end{array}$ & $\begin{array}{l}0.1365 \\
(0.457)\end{array}$ & $\begin{array}{l}9.0499^{*} \\
(33.903)\end{array}$ & 48.8 \\
\hline Taiwan & $\begin{array}{c}0.0126^{*} \\
(109.355)\end{array}$ & $\begin{array}{l}0.5289^{*} \\
(26.917)\end{array}$ & $\begin{array}{l}0.2551^{*} \\
(12.731)\end{array}$ & $\begin{array}{l}-6.9093^{*} \\
(-14.498)\end{array}$ & $\begin{array}{l}5.469^{*} \\
(5.469)\end{array}$ & 31.26 \\
\hline \multicolumn{7}{|c|}{ African Markets } \\
\hline Egypt & $\begin{array}{l}0.0155^{\star} \\
(58.375)\end{array}$ & $\begin{array}{l}-0.0085 \\
(-0.298)\end{array}$ & $\begin{array}{l}-0.1729^{*} \\
(-5.878)\end{array}$ & $\begin{array}{l}6.4267^{\star} \\
(14.887)\end{array}$ & $\begin{array}{l}11.2528^{*} \\
(23.281)\end{array}$ & 37.81 \\
\hline Kenya & $\begin{array}{l}0.0184^{*} \\
(29.95)\end{array}$ & $\begin{array}{l}0.8971^{\star} \\
(33.74)\end{array}$ & $\begin{array}{l}1.1026^{*} \\
(47.58)\end{array}$ & $\begin{array}{c}-0.6401^{*} \\
(-10.2)\end{array}$ & $\begin{array}{l}-1.1881^{*} \\
(-16.74)\end{array}$ & 71.47 \\
\hline Mauritius & $\begin{array}{l}0.0049^{*} \\
(58.96)\end{array}$ & $\begin{array}{l}0.9823^{*} \\
(66.83)\end{array}$ & $\begin{array}{l}0.8588^{*} \\
(40.84)\end{array}$ & $\begin{array}{c}0.1552^{*} \\
(61.1)\end{array}$ & $\begin{array}{l}5.298^{*} \\
(13.77)\end{array}$ & 99.95 \\
\hline Nigeria & $\begin{array}{l}0.0208^{*} \\
(23.833)\end{array}$ & $\begin{array}{l}0.9423^{*} \\
(26.654)\end{array}$ & $\begin{array}{l}0.8198^{*} \\
(46.156)\end{array}$ & $\begin{array}{l}-0.2884^{*} \\
(-5.967)\end{array}$ & $\begin{array}{l}0.4539^{\star} \\
(18.339)\end{array}$ & 79.22 \\
\hline South Africa & $\begin{array}{l}0.0117^{\star} \\
(89.441)\end{array}$ & $\begin{array}{l}0.3971^{\star} \\
(22.551)\end{array}$ & $\begin{array}{l}0.3291^{*} \\
(19.739)\end{array}$ & $\begin{array}{l}-1.1277^{\star} \\
(-3.345)\end{array}$ & $\begin{array}{l}-0.4442 \\
(-1.585)\end{array}$ & 28.42 \\
\hline & & Middle & East Marke & & & \\
\hline Abu Dhabi & $\begin{array}{l}0.0186^{*} \\
(22.69)\end{array}$ & $\begin{array}{c}1.0555^{*} \\
(30.5)\end{array}$ & $\begin{array}{l}1.0001^{*} \\
(41.59)\end{array}$ & $\begin{array}{l}-1.2731^{\star} \\
(-10.98)\end{array}$ & $\begin{array}{l}-0.6939^{*} \\
(-13.28)\end{array}$ & 65.27 \\
\hline Bahrain & $\begin{array}{l}0.0137^{\star} \\
(17.274)\end{array}$ & $\begin{array}{l}0.8204^{*} \\
(28.439)\end{array}$ & $\begin{array}{l}0.7306^{\star} \\
(34.591)\end{array}$ & $\begin{array}{c}-0.2142^{*} \\
(-3.08)\end{array}$ & $\begin{array}{c}0.2238^{\star *} \\
(2.577)\end{array}$ & 65.69 \\
\hline Qatar & $\begin{array}{l}0.0144^{*} \\
(21.083)\end{array}$ & $\begin{array}{l}0.4615^{*} \\
(25.726)\end{array}$ & $\begin{array}{l}0.6045^{*} \\
(31.06)\end{array}$ & $\begin{array}{l}0.0013 \\
(0.044)\end{array}$ & $\begin{array}{l}-0.2901^{*} \\
(-6.085)\end{array}$ & 65.58 \\
\hline Saudi Arabia & $\begin{array}{l}0.0475^{*} \\
(76.395)\end{array}$ & $\begin{array}{l}0.8652^{*} \\
(46.414)\end{array}$ & $\begin{array}{l}0.9149^{*} \\
(80.924)\end{array}$ & $\begin{array}{l}0.1022^{*} \\
(3.012)\end{array}$ & $\begin{array}{l}0.0275^{\star *} \\
(2.272)\end{array}$ & 91.69 \\
\hline UAE & $\begin{array}{l}0.0087^{*} \\
(38.486)\end{array}$ & $\begin{array}{l}0.6048^{*} \\
(23.834)\end{array}$ & $\begin{array}{l}0.5121^{*} \\
(14.191)\end{array}$ & $\begin{array}{l}-2.9479^{\star} \\
(-8.976)\end{array}$ & $\begin{array}{l}-1.1277 \\
(-1.316)\end{array}$ & 3.44 \\
\hline
\end{tabular}

This table reports the estimated coefficient for the model described in Equation (8) $\left(C S A D_{t}=\gamma_{0}+\gamma_{1} \cdot\left|R_{m t}\right| \cdot D_{V o l}+\gamma_{2} \cdot\left|R_{m t}\right| \cdot 1-D_{V o l}+\gamma_{3} \cdot R_{m t}^{2} \cdot D_{V o l}+\gamma_{4} \cdot R_{m t}^{2} 1-D_{V o l}+\varepsilon_{t}\right)$. t-statistics are given in parentheses. $\gamma_{3}$ indicates the presence or absence of herding during high volatility days while. $\gamma_{4}$ indicates the same during low volatility days. ${ }^{*}$ indicates statistical significance at $1 \%$ level. ${ }^{*}$ indicates statistical significance at $5 \%$ level. 
suggested by Economou et al. [27] given in Equation (9).

$$
\begin{aligned}
C S A D_{t}= & \gamma_{0}+\gamma_{1} \cdot\left|R_{m t}\right| \cdot D_{T V}+\gamma_{2} \cdot\left|R_{m t}\right| \cdot\left(1-D_{T V}\right)+\gamma_{3} \cdot R_{m t}^{2} \cdot D_{T V} \\
& +\gamma_{4} \cdot R_{m t}^{2} \cdot\left(1-D_{T V}\right)+\varepsilon_{t}
\end{aligned}
$$

where, $D_{T V}$ is 1 when trading volume of that day is higher than the moving average trading volume of last 30 days else $0 . D_{T V}$ represents the days of high trading volume while $\left(1-D_{T V}\right)$ represents the days of low trading volume. Value of $\gamma_{3}$ and $\gamma_{4}$ indicate the presence or absence of herding during high and low trading volume, respectively. Results of the equation are given in $\mathrm{Ta}$ ble 10 .

Greece and UAE are the two countries which observe herding during high trading volume while observe strong anti-herding behaviour during low trading volume. Portugal, China, India, Taiwan and Nigeria are few counties which shown herding during high trading volume but no herding is found during low trading volume. Some counties like Canada, Chile, Sweden, Australia, Japan and Singapore find strong anti-herding behaviour only in low market volume, which indicates the herding asymmetry in respect of high and low trading volume period.

\subsection{Dynamic Model of Herding}

Static model proposed by Christie and Huang [35] and Chang et al. [11] are provide results which are sensitive towards the change in sample period, frequency and structural breaks. Static model provide the results based on the average and negate the impact of positive and negative shocks [37]. Therefore, it becomes important to study the time varying nature of herding to check its presence and absence during different phases of market. We use Equation (3) again over a rolling window of 240 days (approx. trading days in a year). Under rolling window methodology, if the parameter $\gamma_{2}$ remains constant over a rolling window indicate the stability of the relationship else we can capture the time varying nature of herding. Under a fast time scale short window can be appropriate while under slow time scale longer rolling window is required to capture the signature changes in time series. A small rolling frame may fails to capture the irregular trend while a long rolling window may smooth out the trends, therefore, we take one year as a rolling window to determine the time varying nature of herding. Results are shown in graphs given in Figure 1. Graph line shows the time varying value of $\gamma_{2}$ and dotted line is $\pm 1.96 \mathrm{SE}$.

Figures 1(a)-(e) show the dynamic herding in Brazil, Canada, Chile, Mexico and US respectively. In confirmation of the results of Stavroyiannis and Babalos [37], our results of the rolling window analysis also indicate that expect few instances of negative non-statistically $\gamma_{2}$ all American and Latin American countries are having an anti-herding behaviour at the most of the time. Among all five markets, the no. of instances when $\gamma_{2}$ is negative are comparatively higher in case of US market. 
Table 10. Regression estimates of herding behaviour during high and low trading volume days.

\begin{tabular}{|c|c|c|c|c|c|c|}
\hline Country Name & $\gamma_{0}$ & $\gamma_{1}$ & $\gamma_{2}$ & $\gamma_{3}$ & $\gamma_{4}$ & Adj. $R^{2}$ \\
\hline \multicolumn{7}{|c|}{ American and Latin American Markets } \\
\hline \multirow[t]{2}{*}{ Brazil } & $0.0142^{\star}$ & $0.2225^{\star}$ & 0.0265 & $0.9911^{*}$ & $6.4311^{\star}$ & 37.08 \\
\hline & $(79.724)$ & $(13.394)$ & $(1.098)$ & $(4.048)$ & $(11.279)$ & \\
\hline \multirow[t]{2}{*}{ Canada } & $0.0123^{*}$ & $0.4726^{*}$ & $0.2799^{*}$ & 1.0022 & $7.4188^{*}$ & 36.54 \\
\hline & $(113.535)$ & $(22.252)$ & $(11.04)$ & $(1.672)$ & $(9.471)$ & \\
\hline \multirow[t]{2}{*}{ Chile } & $0.0113^{*}$ & $0.1735^{*}$ & $0.1356^{*}$ & 0.1918 & $2.2247^{\star}$ & 20.36 \\
\hline & $(97.89)$ & $(13.134)$ & $(8.696)$ & $(1.044)$ & $(6.231)$ & \\
\hline \multirow[t]{2}{*}{ Mexico } & $0.0095^{*}$ & $0.4018^{*}$ & $0.2688^{*}$ & $1.9837^{*}$ & $5.7972^{*}$ & 42.6 \\
\hline & $(84.928)$ & $(23.964)$ & $(10.443)$ & $(5.209)$ & $(6.151)$ & \\
\hline \multirow[t]{2}{*}{ United States } & $0.0108^{*}$ & $0.4304^{*}$ & $0.3401^{*}$ & -0.0876 & 0.6285 & 31.26 \\
\hline & $(62.644)$ & $(20.726)$ & $(13.532)$ & $(-0.231)$ & $(1.086)$ & \\
\hline \multicolumn{7}{|c|}{ European Markets } \\
\hline \multirow[t]{2}{*}{ Belgium } & $0.0076^{*}$ & $0.3711^{\star}$ & $0.2964^{*}$ & $1.3974^{*}$ & $3.8902^{*}$ & 40.05 \\
\hline & $(67.502)$ & $(20.797)$ & $(14.745)$ & $(3.456)$ & $(8.143)$ & \\
\hline \multirow[t]{2}{*}{ France } & $0.0089^{*}$ & $0.2176^{*}$ & $0.1066^{*}$ & $0.8715^{*}$ & $3.8094^{*}$ & 25.37 \\
\hline & $(74.682)$ & $(15.125)$ & $(4.918)$ & $(3.277)$ & $(6.131)$ & \\
\hline \multirow[t]{2}{*}{ Germany } & $0.0107^{*}$ & $0.3609^{*}$ & $0.3898^{*}$ & 0.6087 & 0.4819 & 27.17 \\
\hline & $(48.398)$ & $(15.761)$ & $(14.743)$ & $(1.7)$ & $(0.975)$ & \\
\hline \multirow[t]{2}{*}{ Greece } & $0.0139^{*}$ & $0.3806^{*}$ & $0.2349^{*}$ & $-0.5936^{*}$ & $1.617^{*}$ & 97.24 \\
\hline & (114.029) & $(27.37)$ & $(22.84)$ & $(-2.634)$ & $(148.463)$ & \\
\hline \multirow[t]{2}{*}{ Netherland } & $0.0091^{*}$ & $0.3306^{*}$ & $0.2384^{*}$ & $1.8406^{*}$ & $2.9247^{*}$ & 33.92 \\
\hline & $(57.487)$ & $(14.208)$ & $(9.63)$ & $(3.525)$ & $(5.286)$ & \\
\hline \multirow[t]{2}{*}{ Portugal } & $0.0089^{*}$ & $0.4793^{\star}$ & $0.3159^{*}$ & $-2.0539^{\star}$ & 0.9795 & 34.68 \\
\hline & $(84.824)$ & $(32.922)$ & $(13.815)$ & $(-7.115)$ & $(1.154)$ & \\
\hline \multirow[t]{2}{*}{ Russia } & $0.0132^{*}$ & $0.3367^{*}$ & $0.3259^{*}$ & $0.8527^{\star *}$ & $1.1437^{*}$ & 98.8 \\
\hline & $(97.71)$ & $(17.587)$ & $(36.052)$ & $(2.098)$ & $(164.135)$ & \\
\hline \multirow[t]{2}{*}{ Sweden } & $0.0092^{\star}$ & $0.2855^{*}$ & $0.1402^{*}$ & -0.1609 & $2.0139^{*}$ & 23.32 \\
\hline & $(71.83)$ & $(17.32)$ & $(6.941)$ & $(-0.502)$ & $(3.988)$ & \\
\hline \multirow[t]{2}{*}{ Switzerland } & $0.0071^{*}$ & $0.3222^{*}$ & $0.17^{*}$ & $0.8151^{\star}$ & $3.8607^{*}$ & 37.11 \\
\hline & $(63.159)$ & $(32.357)$ & $(8.679)$ & $(2.795)$ & $(7.939)$ & \\
\hline \multirow[t]{2}{*}{ United Kingdom } & $0.0095^{*}$ & $0.2639^{*}$ & $0.1892^{*}$ & $2.2289^{*}$ & $4.3898^{*}$ & 32.06 \\
\hline & $(87.478)$ & $(14.919)$ & $(9.272)$ & $(5.28)$ & $(7.833)$ & \\
\hline \multicolumn{7}{|c|}{ Asian and Asia Pacific Markets } \\
\hline \multirow[t]{2}{*}{ Australia } & $0.0169^{*}$ & $0.5772^{\star}$ & $0.3136^{*}$ & 0.0042 & $12.93^{*}$ & 42.76 \\
\hline & $(178.685)$ & $(30.973)$ & $(11.158)$ & $(0.0008)$ & $(9.825)$ & \\
\hline \multirow[t]{2}{*}{ China } & $0.0119^{*}$ & $0.3909^{*}$ & $0.2508^{*}$ & $-3.2427^{\star}$ & 0.2473 & 23.9 \\
\hline & $(83.051)$ & $(22.397)$ & $(12.945)$ & $(-9.591)$ & $(0.652)$ & \\
\hline \multirow[t]{2}{*}{ Hong Kong } & $0.0101^{*}$ & $0.2697^{\star}$ & $0.1664^{*}$ & $0.5708^{\star}$ & $4.5314^{*}$ & 34.51 \\
\hline & (87.28) & (21.113) & (7.674) & (2.665) & $(6.866)$ & \\
\hline
\end{tabular}




\section{Continued}

\begin{tabular}{|c|c|c|c|c|c|c|}
\hline India & $\begin{array}{l}0.01176^{*} \\
(90.294)\end{array}$ & $\begin{array}{l}0.4361^{\star} \\
(24.953)\end{array}$ & $\begin{array}{l}0.2897^{\star} \\
(17.432)\end{array}$ & $\begin{array}{l}-1.7728^{*} \\
(-5.188)\end{array}$ & $\begin{array}{l}-0.0008 \\
(-0.003)\end{array}$ & 29.33 \\
\hline Japan & $\begin{array}{l}0.0114^{*} \\
(98.442)\end{array}$ & $\begin{array}{l}0.2166^{\star} \\
(18.267)\end{array}$ & $\begin{array}{c}0.0446^{* *} \\
(2.405)\end{array}$ & $\begin{array}{c}-0.0972 \\
(-0.56)\end{array}$ & $\begin{array}{l}3.8817^{*} \\
(7.674)\end{array}$ & 21.5 \\
\hline Malaysia & $\begin{array}{l}0.0074^{*} \\
(98.942)\end{array}$ & $\begin{array}{c}0.417^{*} \\
(45.325)\end{array}$ & $\begin{array}{l}0.2847^{*} \\
(11.258)\end{array}$ & $\begin{array}{l}0.9394^{*} \\
(141.6)\end{array}$ & $\begin{array}{l}9.819^{*} \\
(6.955)\end{array}$ & 99.38 \\
\hline New Zealand & $\begin{array}{l}0.0109^{*} \\
(118.67)\end{array}$ & $\begin{array}{l}0.8494^{*} \\
(36.91)\end{array}$ & $\begin{array}{l}0.9171^{*} \\
(44.85)\end{array}$ & $\begin{array}{l}8.905^{*} \\
(16.63)\end{array}$ & $\begin{array}{l}7.398^{\star} \\
(28.14)\end{array}$ & 72.77 \\
\hline Singapore & $\begin{array}{l}0.0078^{\star} \\
(62.197)\end{array}$ & $\begin{array}{l}0.3679^{*} \\
(18.285)\end{array}$ & $\begin{array}{l}0.1396^{*} \\
(4.455)\end{array}$ & $\begin{array}{l}0.3575 \\
(0.898)\end{array}$ & $\begin{array}{l}8.7717^{*} \\
(7.577)\end{array}$ & 42.29 \\
\hline South Korea & $\begin{array}{c}0.0154^{\star} \\
(114.821)\end{array}$ & $\begin{array}{l}0.3391^{\star} \\
(18.539)\end{array}$ & $\begin{array}{l}0.0683^{*} \\
(4.184)\end{array}$ & $\begin{array}{l}0.7691^{\star *} \\
(2.307)\end{array}$ & $\begin{array}{l}7.6481^{\star} \\
(29.134)\end{array}$ & 45.05 \\
\hline Taiwan & $\begin{array}{c}0.0125^{\star} \\
(107.141)\end{array}$ & $\begin{array}{l}0.5631^{\star} \\
(26.813)\end{array}$ & $\begin{array}{l}0.2889^{\star} \\
(14.826)\end{array}$ & $\begin{array}{l}-7.4257^{\star} \\
(-13.329)\end{array}$ & $\begin{array}{l}0.4334 \\
(0.946)\end{array}$ & 30.12 \\
\hline \multicolumn{7}{|c|}{ African Markets } \\
\hline Egypt & $\begin{array}{l}0.0115^{\star} \\
(53.144)\end{array}$ & $\begin{array}{l}0.1882^{*} \\
(7.598)\end{array}$ & $\begin{array}{l}-0.1554^{*} \\
(-6.622)\end{array}$ & $\begin{array}{l}-0.1758 \\
(-0.458)\end{array}$ & $\begin{array}{l}12.5875 \\
(0.2936)\end{array}$ & 77.17 \\
\hline Kenya & $\begin{array}{l}0.0194^{*} \\
(25.585)\end{array}$ & $\begin{array}{l}0.9517^{\star} \\
(27.749)\end{array}$ & $\begin{array}{l}0.9784^{*} \\
(33.634)\end{array}$ & $\begin{array}{l}-0.6788^{*} \\
(-6.923)\end{array}$ & $\begin{array}{l}-0.6769^{*} \\
(-8.402)\end{array}$ & 71.82 \\
\hline Mauritius & $\begin{array}{l}0.0049^{*} \\
(47.526)\end{array}$ & $\begin{array}{l}1.0938^{*} \\
(38.064)\end{array}$ & $\begin{array}{l}1.0722^{\star} \\
(38.188)\end{array}$ & $\begin{array}{l}-6.0256^{\star} \\
(-6.222)\end{array}$ & $\begin{array}{l}-7.0497^{\star} \\
(-9.226)\end{array}$ & 61.14 \\
\hline Nigeria & $\begin{array}{l}0.0161^{*} \\
(12.72)\end{array}$ & $\begin{array}{l}1.4958^{\star} \\
(20.571)\end{array}$ & $\begin{array}{l}0.9711^{\star} \\
(18.977)\end{array}$ & $\begin{array}{l}-2.6982^{\star} \\
(-8.684)\end{array}$ & $\begin{array}{l}-0.2273 \\
(-1.299)\end{array}$ & 67.5 \\
\hline South Africa & $\begin{array}{l}0.0117^{\star} \\
(89.603)\end{array}$ & $\begin{array}{l}0.3376^{\star} \\
(18.197)\end{array}$ & $\begin{array}{l}0.3818^{\star} \\
(24.143)\end{array}$ & $\begin{array}{l}-0.1846 \\
(-0.511)\end{array}$ & $\begin{array}{l}-0.9397^{\star} \\
(-3.502)\end{array}$ & 28.23 \\
\hline \multicolumn{7}{|c|}{ Middle-East Markets } \\
\hline Abu Dhabi & $\begin{array}{l}0.0204^{*} \\
(21.787)\end{array}$ & $\begin{array}{l}1.0644^{*} \\
(21.607)\end{array}$ & $\begin{array}{l}0.9172^{*} \\
(34.097)\end{array}$ & $\begin{array}{l}-1.5641^{*} \\
(-7.888)\end{array}$ & $\begin{array}{l}-0.6077^{*} \\
(-11.666)\end{array}$ & 65.14 \\
\hline Bahrain & $\begin{array}{l}0.0141^{*} \\
(16.238)\end{array}$ & $\begin{array}{c}0.921^{*} \\
(25.173)\end{array}$ & $\begin{array}{l}0.9065^{\star} \\
(29.124)\end{array}$ & $\begin{array}{l}-0.5685^{\star} \\
(-6.699)\end{array}$ & $\begin{array}{l}-0.5759^{\star} \\
(-5.873)\end{array}$ & 65.23 \\
\hline Qatar & $\begin{array}{l}0.0135^{\star} \\
(18.835)\end{array}$ & $\begin{array}{l}0.5586^{*} \\
(24.083)\end{array}$ & $\begin{array}{l}0.6489^{*} \\
(30.227)\end{array}$ & $\begin{array}{l}-0.1921^{*} \\
(-3.361)\end{array}$ & $\begin{array}{l}-0.4702^{*} \\
(-9.512)\end{array}$ & 64.74 \\
\hline Saudi Arabia & $\begin{array}{l}0.0481^{*} \\
(57.858)\end{array}$ & $\begin{array}{l}0.9265^{\star} \\
(38.731)\end{array}$ & $\begin{array}{l}0.9194^{*} \\
(53.431)\end{array}$ & $\begin{array}{l}0.0017 \\
(0.031)\end{array}$ & $\begin{array}{l}0.0272 \\
(1.849)\end{array}$ & 91.49 \\
\hline UAE & $\begin{array}{l}0.0109^{\star} \\
(44.745)\end{array}$ & $\begin{array}{l}0.5434^{\star} \\
(22.259)\end{array}$ & $\begin{array}{l}0.1369^{*} \\
(4.011)\end{array}$ & $\begin{array}{l}-3.0634^{*} \\
(-9.536)\end{array}$ & $\begin{array}{l}5.9185^{\star} \\
(9.231)\end{array}$ & 39.62 \\
\hline
\end{tabular}

This table reports the estimated coefficient for the model described in Equation (9)

$\left(C S A D_{t}=\gamma_{0}+\gamma_{1} \cdot\left|R_{m t}\right| \cdot D_{T V}+\gamma_{2} \cdot\left|R_{m t}\right| \cdot\left(1-D_{T V}\right)+\gamma_{3} \cdot R_{m t}^{2} \cdot D_{T V}+\gamma_{4} \cdot R_{m t}^{2} \cdot\left(1-D_{T V}\right)+\varepsilon_{t}\right)$. t-statistics are given in parentheses. $\gamma_{3}$ indicates the presence or absence of herding during high volume market while $\gamma_{4}$ indicates the same during low volume market. ${ }^{*}$ indicates statistical significance at $1 \%$ level. ${ }^{* *}$ indicates statistical significance at $5 \%$ level. 


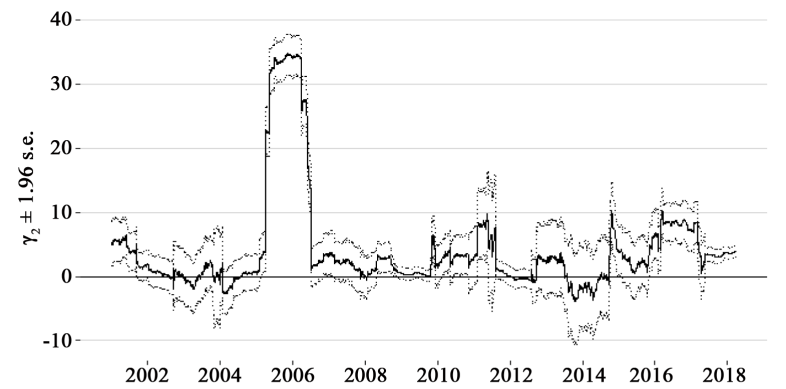

(a)

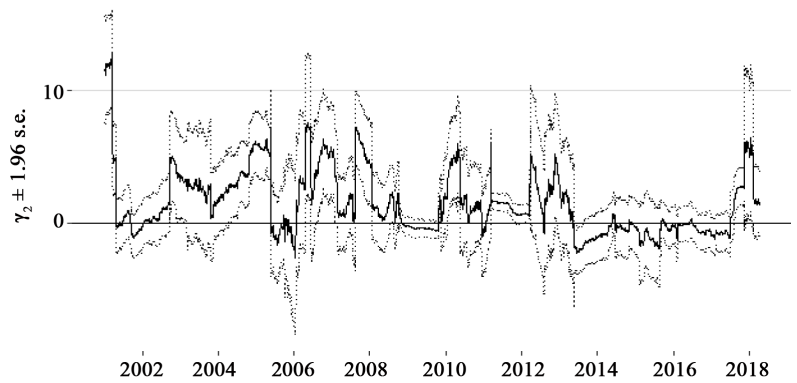

(c)

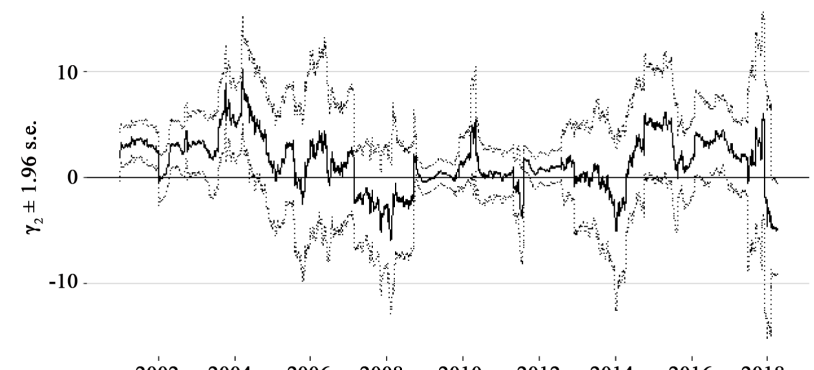

(e)

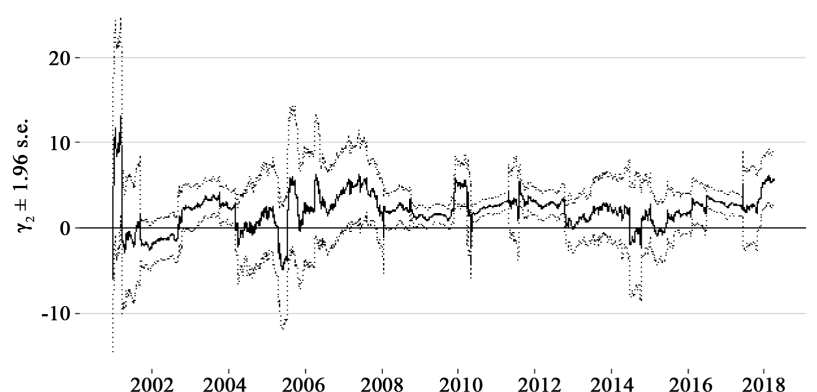

(g)

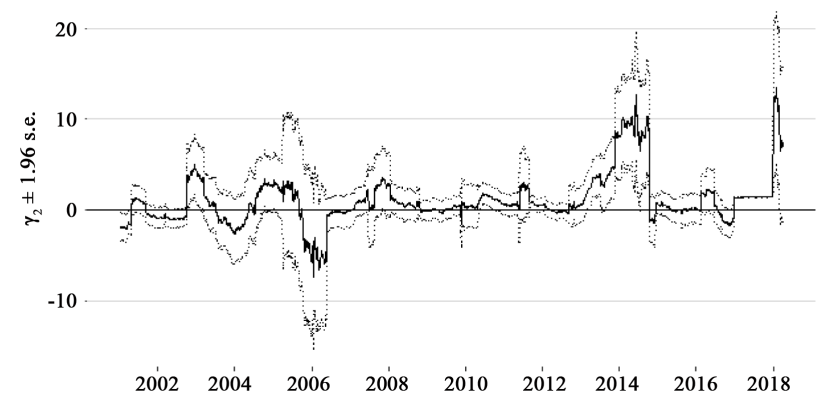

(i)

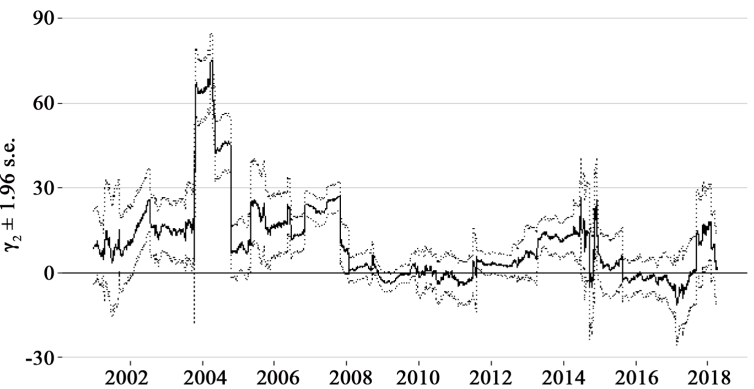

(b)

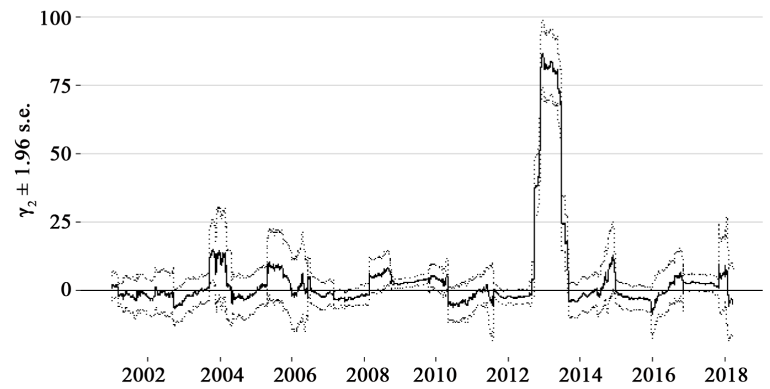

(d)

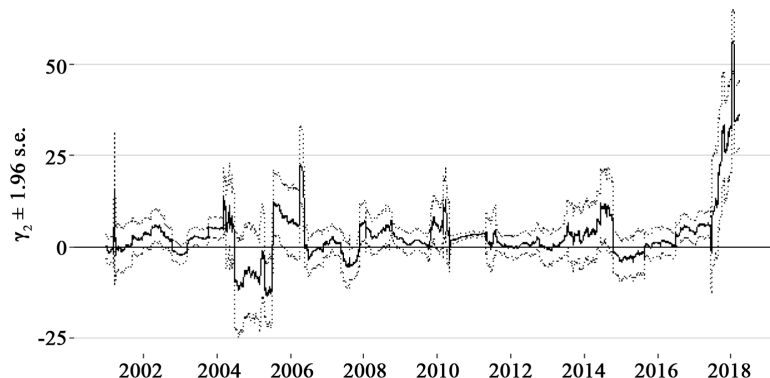

(f)

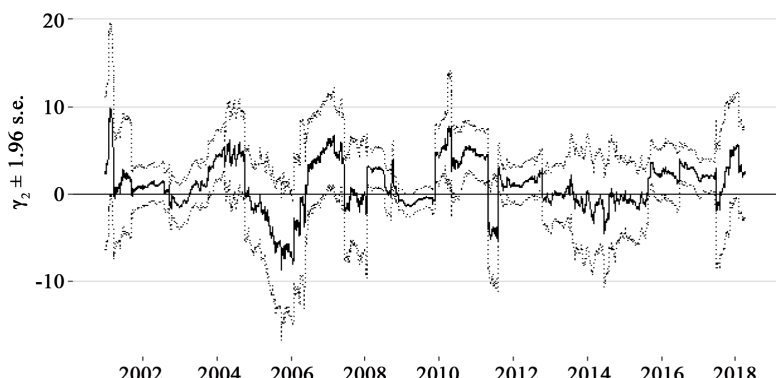

(h)

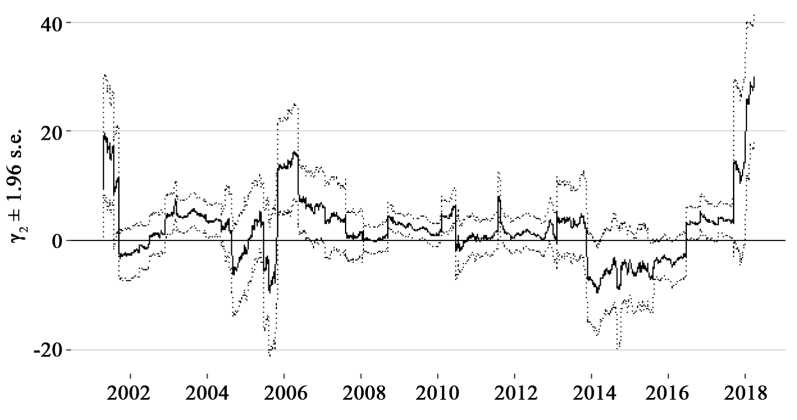

(j) 


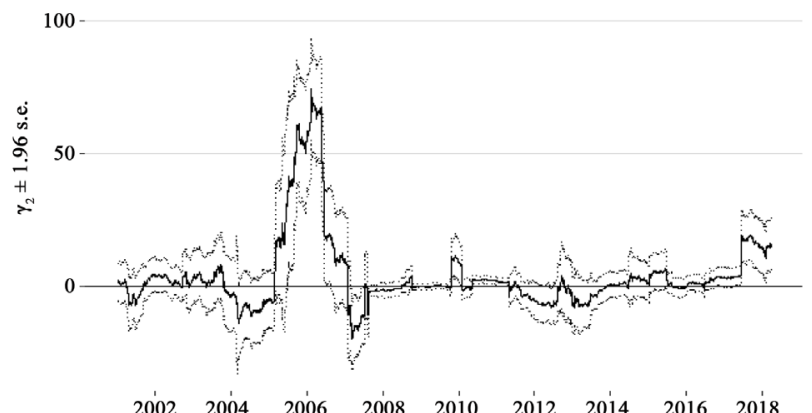

(k)

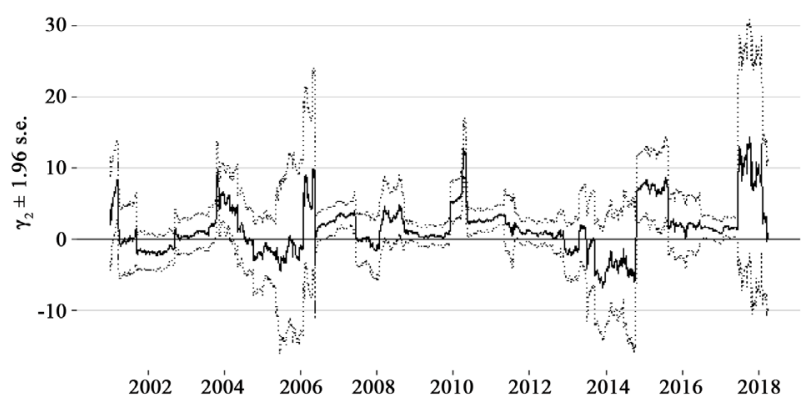

(m)

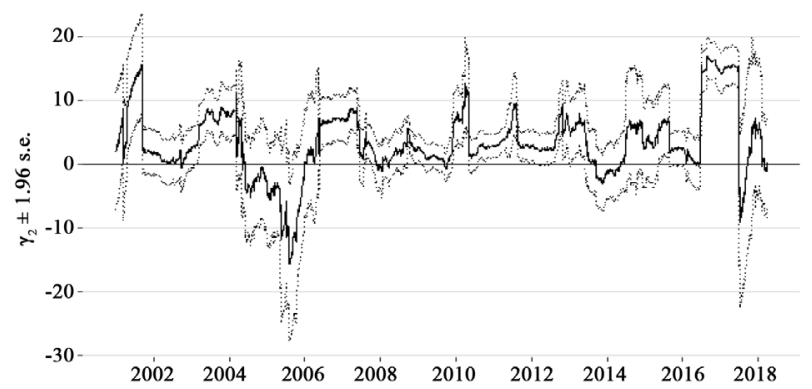

(o)

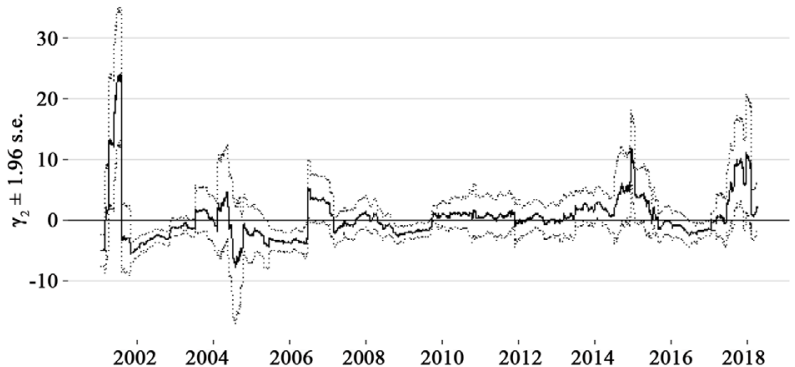

(q)

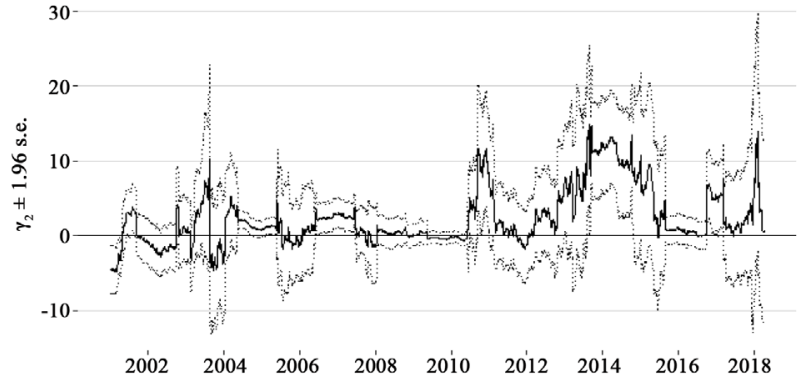

(s)

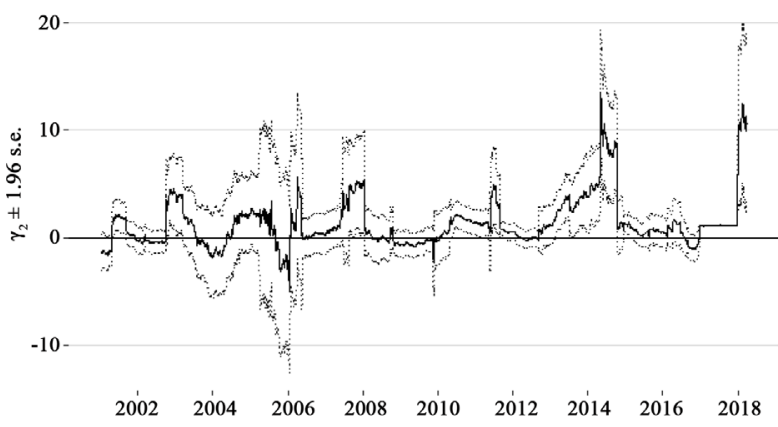

(1)

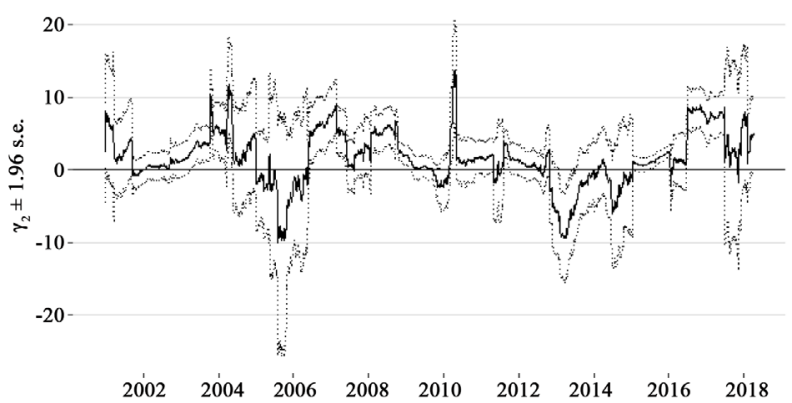

(n)

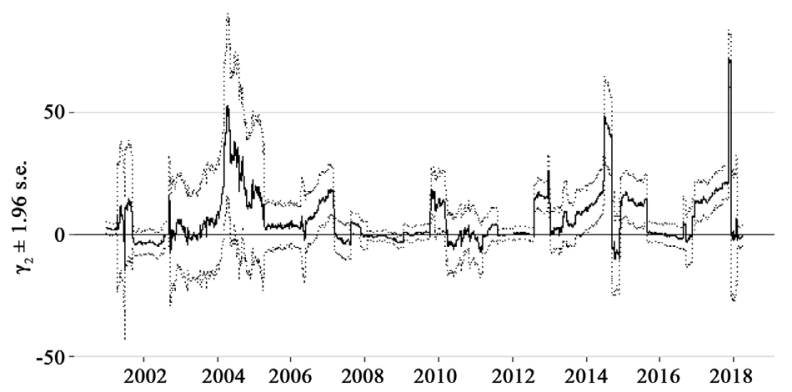

(p)

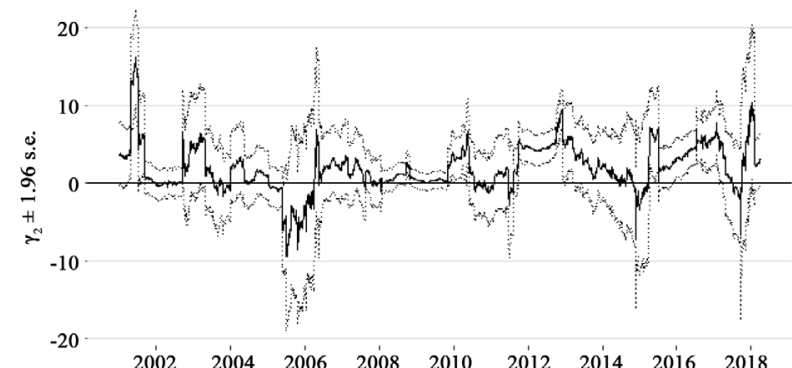

(r)

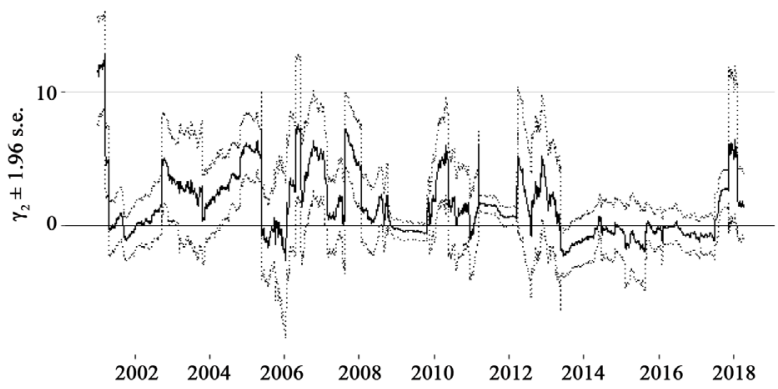

(t) 


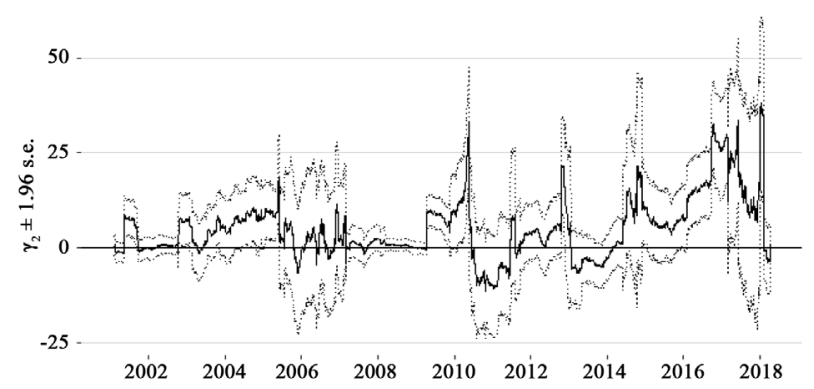

(u)

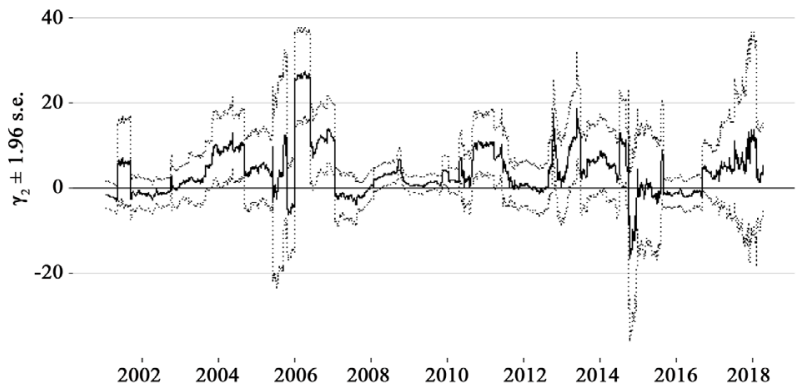

(w)

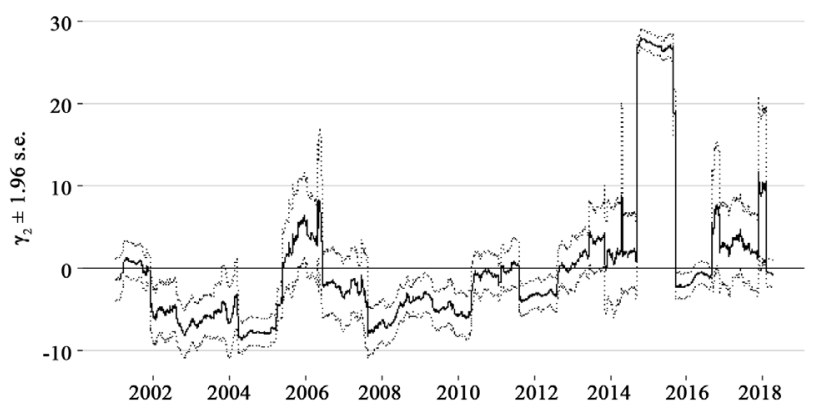

(y)

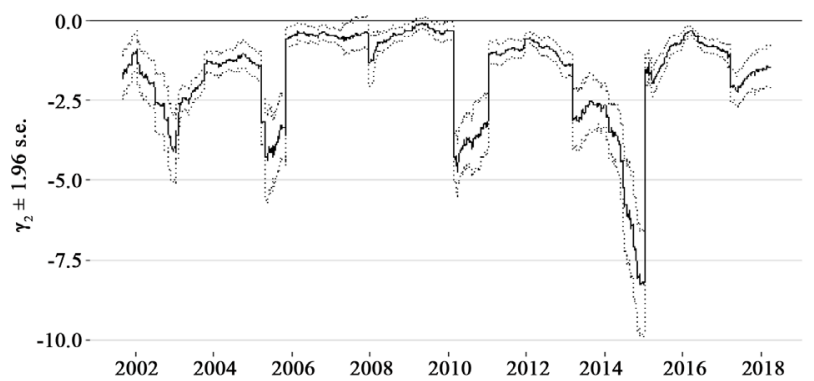

(a)

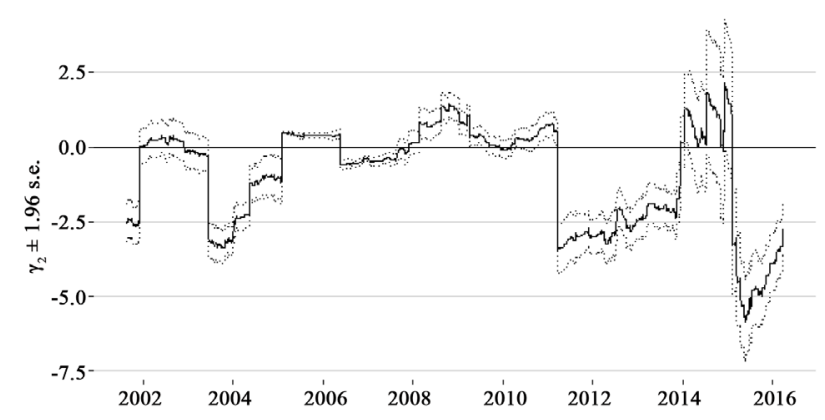

(ac)

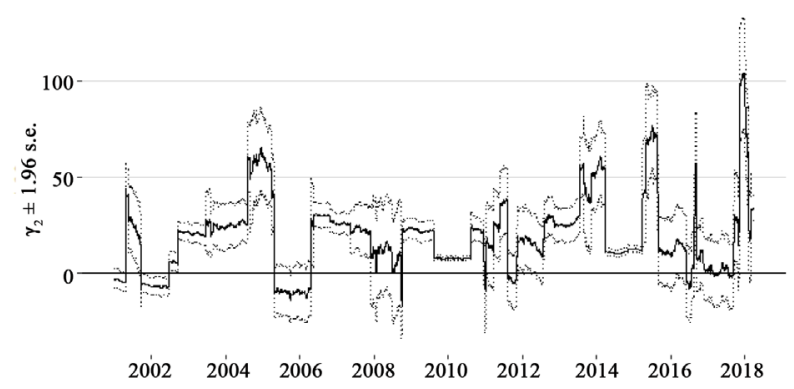

(v)

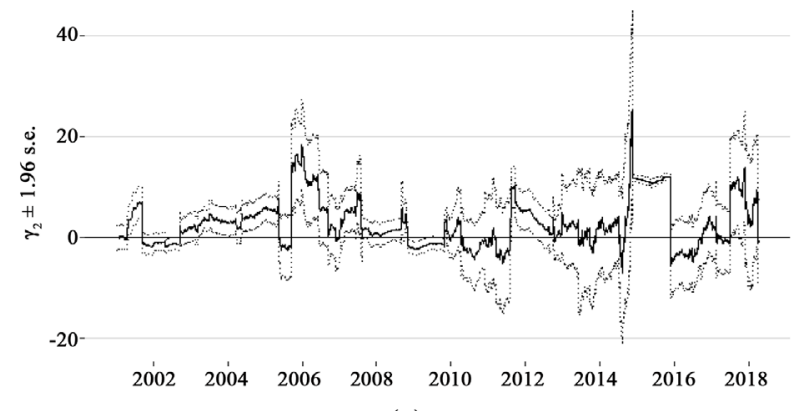

(x)

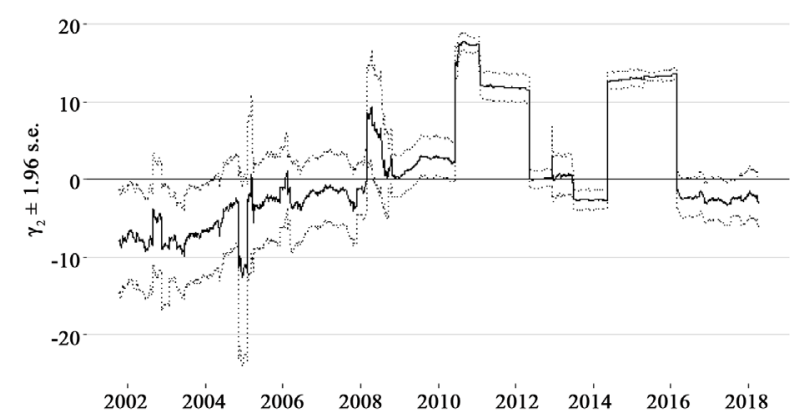

(z)

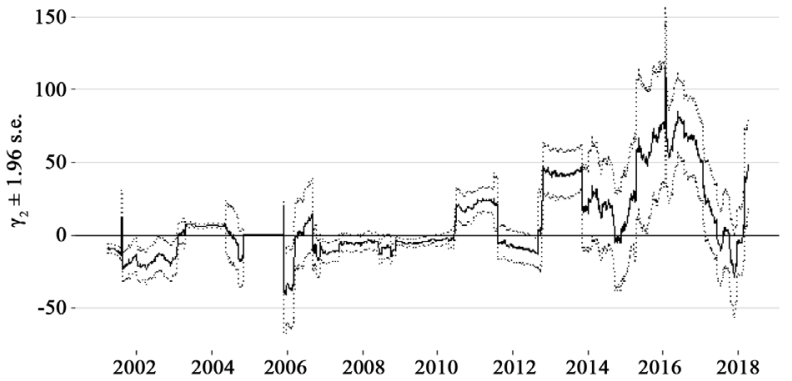

(ab)

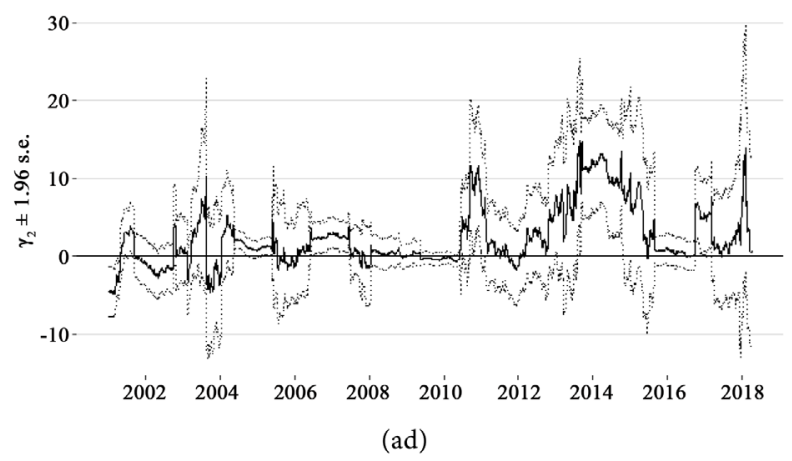



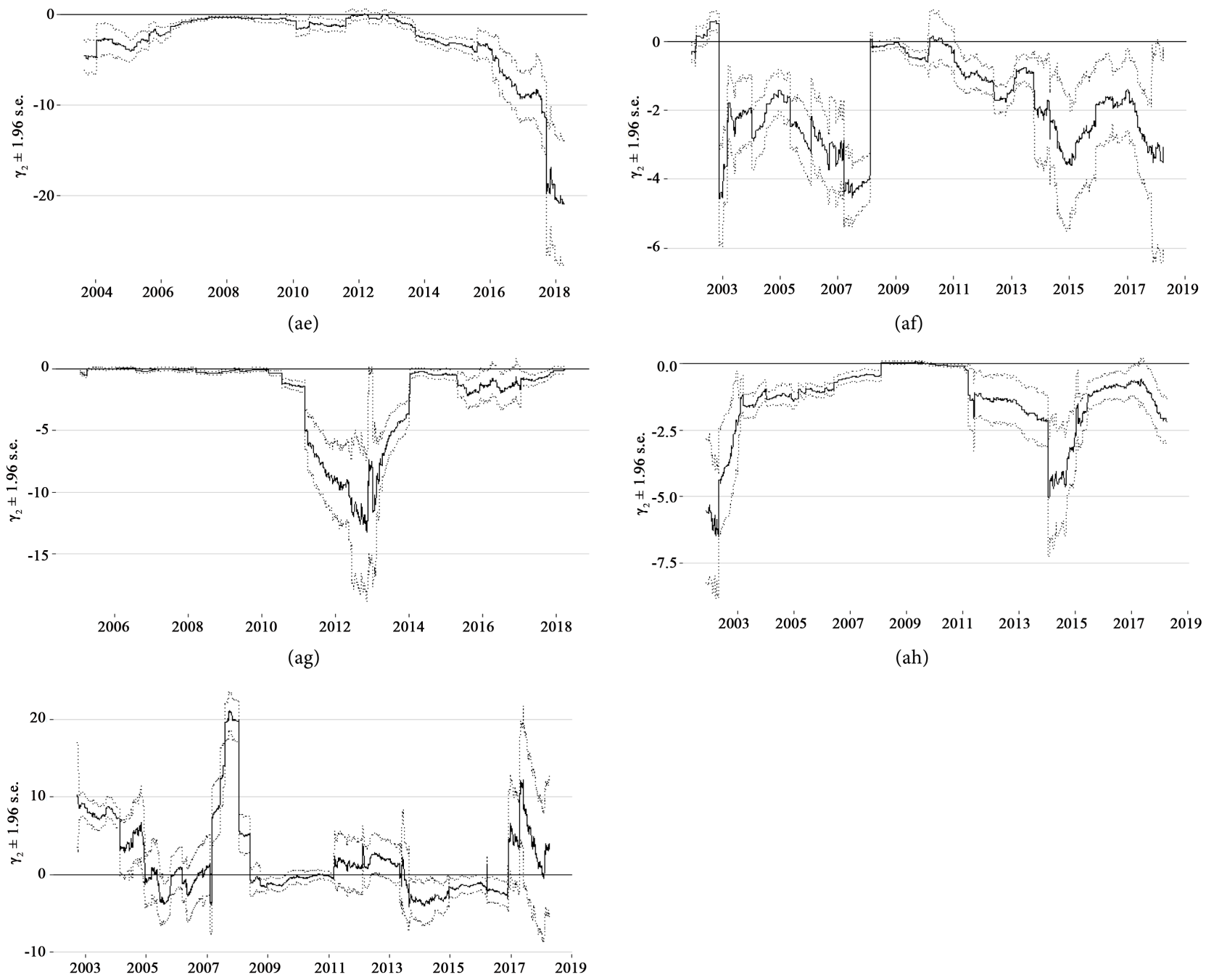

(ah)

(ai)

Figure 1. Rolling window regression results showing the time evolution of $r_{2}$. (a) Brazil; (b) Canada; (c) Chile; (d) Mexico; (e) United States (US); (f) Belgium; (g) France; (h) Germany; (i) Greece; (j) Netherland; (k) Portugal; (l) Russia; (m) Sweden; (n) Switzerland; (o) United Kingdom; (p) Australia; (q) China; (r) Hong Kong; (s) India; (t) Japan; (u) Malaysia; (v) New Zealand; (w) Singapore; (x) South Korea; (y) Taiwan; (z) Egypt; (aa) Kenya; (ab) Mauritius; (ac) Nigeria; (ad) South Africa; (ae) Abu Dhabi; (af) Bahrain; (ag) Qatar; (ah) Saudi Arabia; (ai) UAE.

If we look at the graphs representing European markets, with few exceptions majority of the markets have significant herding during two phases: 2004-2006 and 2014-2016. Both the phases belong to the crisis period in Europe. Looking at Asian and Asia Pacific markets, except for Australia results of other markets demonstrates significant fluctuations between positive and negative value of $\gamma_{2}$ and support the time varying nature of the herding in these markets. Except for Mauritius and South Africa, all sampled African and Middle East markets show the strong herding during entire sample period.

\subsection{State of Economy, Geography Location, Culture and Herding}

Another objective of our study is to relate the state of economy with presence of 
herding, as herding is perceived to be an emerging market phenomenon. We classify all 35 countries on the basis of their state of economy advance or emerging. MSCI classification is used to determine the state of an economy. Data is available at MSCI website (https://www.msci.com/market-classification). We created dummy for developed market $\left(D_{\text {Devp }}\right)$ and emerging markets $\left(D_{\text {Emeg }}\right)$ and run the regression using Equation (10) to determine the effect of state of economy and herding.

$$
\begin{aligned}
C S A D_{t}= & \gamma_{0}+\gamma_{1} \cdot\left|R_{m t}\right| \cdot D_{\text {Devp }}+\gamma_{2} \cdot\left|R_{m t}\right| \cdot D_{\text {Emeg }}+\gamma_{3} \cdot R_{m t}^{2} \cdot D_{\text {Devp }}+\gamma_{4} \cdot R_{m t}^{2} D_{\text {Emeg }}+\varepsilon_{t} \\
= & 0.0185-26.8876\left|R_{m t}\right|-0.3459\left|R_{m t}\right|+26796.053 \cdot R_{m t}^{2}+2.443 \cdot R_{m t}^{2} \\
& \left(\begin{array}{llll}
7.349) & (-1.726) & (-1.234)
\end{array}\right. \\
R^{2}=14.86 \% &
\end{aligned}
$$

Negatively significant values of $\gamma_{3}$ and $\gamma_{4}$ establish the relation of stated of economy with herding but in case of our analysis both the coefficients are positive and insignificant which negate the impact of the state of economy on herding.

It has been observed that selected American, Latin American and European markets except Portugal do not indicate the presence of herding while majority of Asian, African and Middle East markets are observed with herding behaviour. That tempted us to check the impact of geographical location of a country on herding. Justification of the same is given by Chang and Lin [30]. They argue herding is human tendency to follow others action that is very much culture depend and native of the same region usually share culture too. Therefore, it will be interesting to check the impact of geographical location on herding. On the basis of cultural similarity, we classify the countries into two parts: eastern countries and western countries. American, Latin American and European countries are western countries while Asian, Asia Pacific, African and Middle East countries are eastern countries and created dummy for the same as we created for state of the economy and run regression using Equation (11).

$$
\begin{aligned}
C S A D_{t}= & \gamma_{0}+\gamma_{1} \cdot\left|R_{m t}\right| \cdot D_{\text {East }}+\gamma_{2} \cdot\left|R_{m t}\right| \cdot D_{\text {West }}+\gamma_{3} \cdot R_{m t}^{2} \cdot D_{\text {East }}+\gamma_{4} \cdot R_{m t}^{2} D_{\text {West }}+\varepsilon_{t} \\
= & 0.0179-0.3132\left|R_{m t}\right|-20.729\left|R_{m t}\right|+2.1188 \cdot R_{m t}^{2}+19370.28 \cdot R_{m t}^{2} \\
& \left(\begin{array}{llll}
7.533) & (-1.116) & (-1.316) & (0.559)
\end{array}\right. \\
R^{2}=12.11 \% &
\end{aligned}
$$

Negatively significant values of $\gamma_{3}$ and $\gamma_{4}$ establish the relation of geographical location with herding but in case of our analysis both the coefficients are positive and insignificant which negate the impact of the culture on herding. In order to strengthen our finding related culture, we adopt the data on national culture indexes proposed by Hofsted [43] on the five dimensions of national nature: Power distance (PHI), individualism (IDV), masculinity (MAS), uncer- 
tainty avoidance (UAI), and long-term orientation (LTO) and try to related this dimension with herding. We don't have data on cultural index for Mauritius, Abhu Dhabi, Bahrain and Qatar therefore, run the regression with 31 countries only. Similar to Chang and Lin [30], we conduct a multinomial regression analysis using following equation:

$$
\begin{aligned}
& D_{\gamma_{2}, i}=\alpha_{1}+\alpha_{2} \mathrm{PHI}+\alpha_{3} \mathrm{IDV}+\alpha_{4} \mathrm{MAS}+\alpha_{5} \mathrm{UAI}+\alpha_{6} \mathrm{LTO}+\varepsilon_{i} \\
& =0.7861+0.0029 \mathrm{PHI}-0.0058 \mathrm{IDV}+0.0039 \mathrm{MAS}-0.0073 \mathrm{UAI}-0.0053 \mathrm{LTO}(12) \\
& \begin{array}{llll}
(0.633) & (0.264) & (-0.617) & (0.440)
\end{array}
\end{aligned}
$$

where is $D_{\gamma_{2}, i} 1$ if country have significant negative value of $\gamma_{2}$ in Equation (3), -1 if country have a significant positive value of $\gamma_{2}$ and 0 if a country have insignificant value of $\gamma_{2}$ irrespective of sign.

According to Equation (12), if coefficient $\alpha_{1}, \alpha_{2}, \alpha_{3}, \alpha_{4}$, or $\alpha_{5}$ is statistically and significantly different from zero, it indicates that a national cultural index significantly influences investors' herding behavior. But in our analysis all coefficients are insignificant and again confirm our earlier findings that the culture does not influence the herding tendency of investors in a country.

\section{Conclusions}

In our work, we try to analyse the presence of herding in world markets. We contribute to the existing literature in many ways. We select 35 world markets from different corners of the word and extracted the daily data of all constituents stocks of the representative indices of these markets over most recent period ranging from Jan. 2000 to Apr. 2018. Second. We apply all alternate methodologies available to detect market wide herding. In our knowledge, we are the first one to determine the impact of state of economy and region on herding.

Applying different methodologies static and time varying, and find that only 11 markets out of 35 exhibit significant herding behaviour. These markets majorly belong to Asia, Africa and Middle East. Asymmetric behaviour of herding is observed as herding indicator become bigger and significant during down market. Those countries, which exhibit overall herding, are found with high and significant herding measure in case of high volatility and similar is true in case of high trading volume. Explanation of the same may be found in prospect theory as human seek more conformity on the time of losses and volatility. Rolling window analysis reveals that anti-herding become more intense in case of crisis period as we can see that during 2008-09 subprime crisis period, majority of the countries are showing either positive or insignificant negative value of herding indicator. We also try to analyse the impact of state of economy, geography location and culture on herding and our analysis do not observe any impact of these variable on herding.

Herding is a psychological phenomenon, which intends an investor to mimic other investment actions. As a result that may destabilize a country stock market and increase the fragility of the financial system. Based on the empirical results 
of our study, we find that investor herding tendency varies among stock markets and that does not depend on the state of economy, as in our analysis US market also detected with herding during down market while other emerging markets are not. We believe it depends more on the rule \& regulation and financial infrastructure of a country. Therefore, our finding may provide an insight to the govt. of these countries to improve the market microstructure and regulations to make the market herding free. International investors are also takes clue from presence of herding to build an international portfolio.

\section{Conflicts of Interest}

The authors declare no conflicts of interest regarding the publication of this paper.

\section{References}

[1] Pochea, M.-M., Filip, A.-M. and Pece, A.-M. (2017) Herding Behavior in CEE Stock Markets under Asymmetric Conditions: A Quantile Regression Analysis. Journal of Behavioral Finance, 18, 400-416. https://doi.org/10.1080/15427560.2017.1344677

[2] Shiller, R.J. (1990) Investor Behavior in the October 1987 Stock Market Crash: Survey Evidence in Market Volatility. MIT Press, Cambridge, MA.

[3] Folkerts-Landau, D. and Garber, P.M. (1999) The New Financial Architecture: A Threat to the Markets? Global Markets Research, 2. Deutsche Bank AG.

[4] Persaud, A. (2000) Sending the Herd off the Cliff Edge: The Disturbing Interaction between Herding and Market-Sensitive Risk Management Practices. The Journal of Risk Finance, 2, 59-65.

[5] Blasco, N., Corredor, P. and Ferreruela, S. (2012) Does Herding Affect Volatility? Implications for the Spanish Stock Market. Quantitative Finance, 12, 311-327. https://doi.org/10.1080/14697688.2010.516766

[6] Mobarek, A., Mollah, S. and Keasey, K. (2014) A Cross-Country Analysis of Herd Behavior in Europe. Journal of International Financial Markets, Institutions and Money, 32, 107-127. https://doi.org/10.1016/j.intfin.2014.05.008

[7] Chattopadhyay, M., Garg, A.K. and Mitra, S.K. (2017) Herding by Foreign Institutional Investors: An Evidential Exploration for Persistence and Predictability. Journal of Behavioral Finance, 19, 73-88. https://doi.org/10.1080/15427560.2017.1373282

[8] Froot, K., Scharfstein, D. and Stein, J. (1992) Herd on the Street: Informational Efficiencies in a Market with Short-Term Speculation. Journal of Finance, 47, 1461-1484. https://doi.org/10.1111/j.1540-6261.1992.tb04665.x

[9] Gelos, R.G. and Wei, S.-J. (2005) Transparency and International Portfolio Holdings. Journal of Finance, 60, 2987-3020. https://doi.org/10.1111/j.1540-6261.2005.00823.x

[10] Ali, S., Shahzad, S.J.H., Raja, N. and Al-Yahyaee, K.H. (2018) Stock Market Efficiency: A Comparative Analysis of Islamic and Conventional Stock Markets. Physica $A, 503,139-153$. https://doi.org/10.1016/j.physa.2018.02.169

[11] Chang, E.C., Cheng, J.W. and Khorana, A. (2000) An Examination of Herd Behavior in Equity Markets: An International Perspective. Journal of Banking and Finance, 24, 1651-1679. https://doi.org/10.1016/S0378-4266(99)00096-5 
[12] Barbara, A. and Ornelas, J.R.H. (2006) Herding Behaviour by Equity Foreign Investors on Emerging Markets. Working Papers Series 125, Central Bank of Brazil, Research Department.

[13] Holmes, P., Kallinterakis, V. and Ferreira, M.P.L. (2013) Herding in a Concentrated Market: A Question of Intent. European Financial Management, 19, 497-520. https://doi.org/10.1111/j.1468-036X.2010.00592.x

[14] Economou, F., Katsikas, E. and Vickers, G. (2016) Testing for Herding in the Athens Stock Exchange during the Crisis Period. Finance Research Letters, 18, 334-341. https://doi.org/10.1016/j.frl.2016.05.011

[15] Yao, J., Ma, C.C. and He, W.P. (2014) Investor Herding Behaviour of Chinese Stock Market. International Review of Economics \& Finance, 29, 12-29. https://doi.org/10.1016/j.iref.2013.03.002

[16] Sharma, S.S., Narayan, P. and Thuraisamy, K. (2015) Time-Varying Herding Behaviour, Global Financial Crisis, and the Chinese Stock Market. Review of Pacific Basin Financial Markets and Policies, 18, 1550-1559. https://doi.org/10.1142/S0219091515500095

[17] Bhaduri, S.N. and Mahapatra, S.D. (2013) Applying an Alternative Test of Herding Behavior: A Case Study of the Indian Stock Market. Journal of Asian Economics, 25, 43-52. https://doi.org/10.1016/j.asieco.2013.02.001

[18] Lu, Y.-C., Fan, H. and Nieh, C.-C. (2012) The Price Impact of Foreign Institutional Herding on Large-Size Stocks in the Taiwan Stock Market. Review Quantitative Finance and Accounting, 39, 189-208. https://doi.org/10.1007/s11156-011-0244-1

[19] Gavriilidis, K., Kallinterakis, V. and Leite-Ferreira, M.P. (2013) Institutional Industry Herding: Intentional or Spurious? Journal of International Financial Markets, Institutions and Money, 26, 192-214. https://doi.org/10.1016/j.intfin.2013.05.008

[20] Wylie, S. (2005) Fund Manager Herding: A Test of the Accuracy of Empirical Results Using U.K. Data. The Journal of Business, 78, 381-403.

[21] Galariotis, E.C., Rong, W. and Spyrou, S.I. (2015) Herding on Fundamental Information: A Comparative Study. Journal of Banking \& Finance, 50, 589-598. https://doi.org/10.1016/j.jbankfin.2014.03.014

[22] Kremer, S. and Nautz, D. (2013) Causes and Consequences of Short-Term Institutional Herding. Journal of Banking and Finance, 37, 1676-1686. https://doi.org/10.1016/j.jbankfin.2012.12.006

[23] Bensaida, A. (2017) Herding Effect on Idiosyncratic Volatility in U.S. Industries. Finance Research Letters, 23, 121-132. https://doi.org/10.1016/j.frl.2017.03.001

[24] Fang, H., Shen, C.-H. and Lee, Y.-H. (2017) The Dynamic and Asymmetric Herding Behavior of US Equity Fund Managers in the Stock Market. International Review of Economics \& Finance, 49, 353-369. https://doi.org/10.1016/j.iref.2016.12.012

[25] Blasco, N. and Ferreruela, S. (2008) Testing Intentional Herding in Familiar Stocks: An Experiment in an International Context. Journal of Behavioral Finance, 9, 72-84. https://doi.org/10.1080/15427560802093654

[26] Chiang, T.C. and Zheng, D. (2010) An Empirical Analysis of Herd Behavior in Global Stock Markets. Journal of Banking \& Finance, 34, 1911-1921. https://doi.org/10.1016/j.jbankfin.2009.12.014

[27] Economou, F., Kostakis, A. and Philippas, N. (2011) Cross-Country Effects in Herding Behaviour: Evidence from Four South European Markets. Journal of International Financial Markets, Institutions and Money, 21, 443-460. 
https://doi.org/10.1016/j.intfin.2011.01.005

[28] Gebka, B. and Wohar, M.E. (2013) International Herding: Does It Differ across Sectors? Journal of International Financial Markets, Institutions and Money, 23, 55-84. https://doi.org/10.1016/j.intfin.2012.09.003

[29] Balcilar, M., Demirer, R. and Hammoudeh, S. (2014) What Drives Herding in Oil-Rich, Developing Stock Markets? Relative Roles of Own Volatility and Global Factors. The North American Journal of Economics and Finance, 29, 418-440.

https://doi.org/10.1016/j.najef.2014.06.009

[30] Chang, C.H. and Lin, S.J. (2015) The Effects of National and Behavioral Pitfalls on Investors' Decision-Making: Herding Behavior in International Stock Markets. International Review of Economics and Finance, 37, 380-392. https://doi.org/10.1016/j.iref.2014.12.010

[31] Economou, F., Gavriilidis, K., Goyal, A. and Kallinterakis, V. (2015) Herding Dynamics in Exchange Groups: Evidence from Euronext. Journal of International Financial Markets, Institutions and Money, 34, 228-244.

https://doi.org/10.1016/j.intfin.2014.11.013

[32] Guney, Y., Kallinterakis, V. and Komba, G. (2017) Herding in Frontier Markets: Evidence from African Stock Exchanges. Journal of International Financial Markets, Institutions and Money, 47, 152-175. https://doi.org/10.1016/j.intfin.2016.11.001

[33] Zheng, D., Li, H. and Chiang, T.C. (2017) Herding within Industries: Evidence from Asian Stock Markets. International Review of Economics \& Finance, 51, 487-509. https://doi.org/10.1016/j.iref.2017.07.005

[34] Kabir, M.H. and Shakur, S. (2018) Regime-Dependent Herding Behavior in Asian and Latin American Stock Markets. Pacific-Basin Finance Journal, 47, 60-78. https://doi.org/10.1016/j.pacfin.2017.12.002

[35] Christie, W.G. and Huang, R.D. (1995) Following the Pied Piper: Do Individual Return Herd around the Market? Financial Analysts Journal, 51, 31-37. https://doi.org/10.2469/faj.v51.n4.1918

[36] Tan, L., Chiang, T.C., Mason, J.R. and Nelling, E. (2008) Herding Behavior in Chinese Stock Markets: An Examination of A and B Shares. Pacific-Basin Finance Journal, 16, 61-77. https://doi.org/10.1016/j.pacfin.2007.04.004

[37] Stavroyiannis, S. and Babalos, V. (2017) Herding, Faith-Based Investments and the Global Financial Crisis: Empirical Evidence from Static and Dynamic Models. Journal of Behavioral Finance, 18, 478-489. https://doi.org/10.1080/15427560.2017.1365366

[38] Chiang, T., Tan, L., Li, J. and Nelling, E. (2013) Dynamic Herding Behavior in Pacific-Basin Markets: Evidence and Implications. Multinational Finance Journal, 17, 165-200. https://doi.org/10.17578/17-3/4-3

[39] De Long, J.B., Shleifer, A., Summers, L.H. and Waldmann, R.J. (1990) Positive Feedback Investment Strategies and Destabilizing Rational Speculation. The Journal of Finance, 45, 379-395. https://doi.org/10.1111/j.1540-6261.1990.tb03695.x

[40] Corsetti, G., Pericoli, M. and Sbracia, M. (2005) "Some Contagion, Some Interdependence": More Pitfalls in Tests of Financial Contagion. Journal of International Money and Finance, 24, 1177-1199. https://doi.org/10.1016/j.jimonfin.2005.08.012

[41] Litimi, H., BenSaïda, A. and Bouraoui, O. (2016) Herding and Excessive Risk in the American Stock Market: A Sectoral Analysis. Research in International Business and Finance, 38, 6-21. https://doi.org/10.1016/j.ribaf.2016.03.008 
[42] Hwang, S. and Salmon, M. (2004) Market Stress and Herding. Journal of Empirical Finance, 11, 585-616. https://doi.org/10.1016/j.jempfin.2004.04.003

[43] Hofstede, G. (2003) Culture's Consequences: Comparing Values, Behaviors, Institutions and Organizations across Nations. Sage Publications. 